\title{
Nitrile Biotransformations for the Efficient Synthesis of Highly Enantiopure 1-Arylaziridine-2-carboxylic Acid Derivatives and Their Stereoselective Ring Opening Reactions
}

Jin-Yuan Wang, De-Xian Wang, Qi-Yu Zheng, Zhi-Tang Huang, Mei-Xiang Wang*

Beijing National Laboratory for Molecular Sciences, Laboratory of Chemical Biology, Institute of Chemistry, Chinese Academy of Sciences, Beijing 100080, China

\section{mxwang@iccas.ac.cn}

Table of Contents

1. General Experimental Methods. .......................................S1

2. Spectroscopic data of starting materials prepared........................S2

3. Full characterization of all products...................................S3

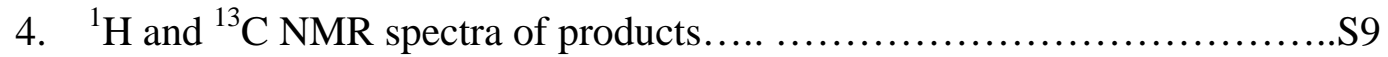

5. HPLC analysis of products. ......................................... 58

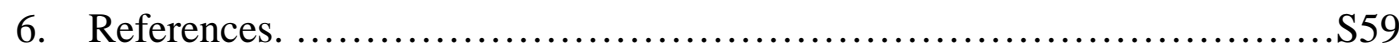

\section{General Experimental Methods.}


Both melting points and boiling points are uncorrected. The racemic nitriles 1a-h were prepared following a literature methods ${ }^{1}$. Enantiomeric excess values of all compounds were obtained from HPLC analyses.

\section{Spectroscopic data of compounds prepared.}

(士)-1-Phenylaziridine-2-carbonitrile (1a): oil (lit. $\left.{ }^{1}\right) ;{ }^{1} \mathrm{H} \mathrm{NMR}\left(300 \mathrm{MHz}, \mathrm{CDCl}_{3}\right.$, TMS) $\delta$ 6.99-7.32 (m, 5H), 2.75 (dd, J = 3.0 Hz, $6.0 \mathrm{~Hz}, 1 \mathrm{H}), 2.69$ (d, J = 3.0 Hz, 1H), $2.47(\mathrm{~d}, \mathrm{~J}=6.0 \mathrm{~Hz}, 1 \mathrm{H}) ; \mathrm{IR}(\mathrm{KBr}) \vee 2247.6 \mathrm{~cm}^{-1}(\mathrm{CN})$.

( \pm )-1-(4-Fluorophenyl)aziridine-2-carbonitrile (1b): oil $\quad$ (lit. $\left.{ }^{1}\right) ; \quad{ }^{1} \mathrm{H} \quad \mathrm{NMR}$ (300MHz, $\left.\mathrm{CDCl}_{3}, \mathrm{TMS}\right) \delta$ 6.97-7.03 (m, 4H), 2.75 (br, 1H), 2.66 (d, J = 2.6 Hz, 1H), $2.46(\mathrm{~d}, \mathrm{~J}=5.7 \mathrm{~Hz}, 1 \mathrm{H}) ; \mathrm{IR}(\mathrm{KBr}) \vee 2248.6 \mathrm{~cm}^{-1}(\mathrm{CN})$.

( \pm )-1-(4-Chlorophenyl)aziridine-2-carbonitrile (1c): $\mathrm{mp} \quad 55-56^{\circ} \mathrm{C}$ (lit. ${ }^{1} \mathrm{mp}$ 55-56 ${ }^{\circ} \mathrm{C}$ racemic); ${ }^{1} \mathrm{H}$ NMR (300MHz, $\left.\mathrm{CDCl}_{3}, \mathrm{TMS}\right) \delta 7.25$ (d, J = 9.7Hz, 2H), 6.95 (d, J = 9.7Hz, 2H), 2.76 (dd, J = 3.0 Hz, 6.0 Hz, 1H), 2.67 (d, J = 3.0 Hz, 1H), 2.47 (d, $\mathrm{J}=6.0 \mathrm{~Hz}, 1 \mathrm{H}) ; \mathrm{IR}(\mathrm{KBr}) \vee 2248.6 \mathrm{~cm}^{-1}(\mathrm{CN})$.

( \pm )-1-(4-Bromophenyl)aziridine-2-carbonitrile (1d): mp 93-94 ${ }^{\circ} \mathrm{C} ;{ }^{1} \mathrm{H} \quad \mathrm{NMR}$ (300MHz, $\left.\mathrm{CDCl}_{3}, \mathrm{TMS}\right) \delta 7.38(\mathrm{~d}, \mathrm{~J}=9.6 \mathrm{~Hz}, 2 \mathrm{H}), 6.92(\mathrm{~d}, \mathrm{~J}=9.6 \mathrm{~Hz}, 2 \mathrm{H}), 2.76$ (dd, $\mathrm{J}=2.5 \mathrm{~Hz}, 6.0 \mathrm{~Hz}, 1 \mathrm{H}), 2.68(\mathrm{~d}, \mathrm{~J}=2.5 \mathrm{~Hz}, 1 \mathrm{H}), 2.47(\mathrm{~d}, \mathrm{~J}=6.0 \mathrm{~Hz}, 1 \mathrm{H}) ;{ }^{13} \mathrm{C} \mathrm{NMR}$ (75MHz, $\left.\mathrm{CDCl}_{3}, \mathrm{TMS}\right) \delta 149.3,132.4,122.3,117.1,117.0,33.5,24.2$; IR (KBr) $v$

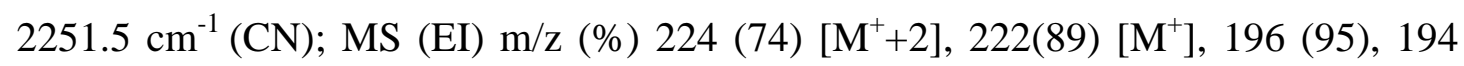
(100), 184 (57), 182 (55), 171 (72), 169 (60), 157 (57), 155 (59); Anal. Calcd for $\mathrm{C}_{9} \mathrm{H}_{7} \mathrm{BrN}_{2}$ : C, 48.46; H, 3.16; N, 12.56; Found: C, 48.47; H, 3.10; N, 12.30. 
( \pm )-1-(4-Methoxyphenyl)aziridine-2-carbonitrile (1e): $\mathrm{mp} \quad 85-87^{\circ} \mathrm{C} \quad$ (lit. $^{1}$ oil racemic); ${ }^{1} \mathrm{H}$ NMR (300MHz, $\left.\mathrm{CDCl}_{3}, \mathrm{TMS}\right) \delta 6.93(\mathrm{~d}, \mathrm{~J}=9.9 \mathrm{~Hz}, 2 \mathrm{H}), 6.83(\mathrm{~d}, \mathrm{~J}=$ 9.9Hz, 2H), 3.77 (s, 3H), 2.68 (br, 1H), 2.64 (br,1H), 2.43 (br,1H); IR (KBr) v 2246.7 $\mathrm{cm}^{-1}(\mathrm{CN})$.

( \pm )-1-p-Tolylaziridine-2-carbonitrile (1f): oil (lit. $\left.{ }^{1}\right) ;{ }^{1} \mathrm{H}$ NMR (300MHz, $\mathrm{CDCl}_{3}$, TMS) $\delta 7.08(\mathrm{~d}, \mathrm{~J}=8.1 \mathrm{~Hz}, 2 \mathrm{H}), 6.90(\mathrm{~d}, \mathrm{~J}=8.1 \mathrm{~Hz}, 2 \mathrm{H}), 2.69$ (dd, J = 2.3 Hz, $5.9 \mathrm{~Hz}$, 1H), 2.64 (d, J = $2.3 \mathrm{~Hz}, 1 \mathrm{H}), 2.43$ (d, J = $5.9 \mathrm{~Hz}, 1 \mathrm{H}), 2.29$ (s, 1H); IR (KBr) v $2247.6 \mathrm{~cm}^{-1}(\mathrm{CN})$.

( \pm )-1-m-Tolylaziridine-2-carbonitrile (1g): oil; ${ }^{1} \mathrm{H}$ NMR (300MHz, $\left.\mathrm{CDCl}_{3}, \mathrm{TMS}\right)$ \& 7.14-7.19 (m, 1H), 6.83-6.94 (m, 3H), 2.71 (dd, J = 3.1 Hz, 6.0 Hz, 1H), 2.66 (dd, J $=1.1 \mathrm{~Hz}, 3.1 \mathrm{~Hz}, 1 \mathrm{H}), 2.44(\mathrm{dd}, \mathrm{J}=0.9 \mathrm{~Hz}, 6.0 \mathrm{~Hz}, 1 \mathrm{H}), 2.32(\mathrm{~s}, 3 \mathrm{H}) ;{ }^{13} \mathrm{C} \mathrm{NMR}$ (75MHz, $\left.\mathrm{CDCl}_{3}, \mathrm{TMS}\right) \delta 150.4,139.4,129.2,125.1,121.2,117.6,117.5,33.4,24.0$, 21.3; IR (KBr) v $2247.6 \mathrm{~cm}^{-1}(\mathrm{CN})$; MS (ESI) m/z (\%) 159.0(100) [M+H $\left.{ }^{+}\right]$; Anal. Calcd for $\mathrm{C}_{10} \mathrm{H}_{10} \mathrm{~N}_{2}$ : C, 75.92; H, 6.37; N, 17.71. Found: C, 75.80; H, 6.32; N, 17.54 .

( \pm )-1-o-Tolylaziridine-2-carbonitrile (1g): oil; ${ }^{1} \mathrm{H}$ NMR $\left(300 \mathrm{MHz}, \mathrm{CDCl}_{3}, \mathrm{TMS}\right)$ ઈ 7.08-7.19 (m, 3H), 6.83 (d, J = 7.8 Hz, 1H), 2.78 (dd, J = 0.8 Hz, 3.0 Hz, 1H), 2.65 (br, 1H), 2.51 (d, J = $6.1 \mathrm{~Hz}, 1 \mathrm{H}) 2.43(\mathrm{~s}, 3 \mathrm{H}) ;{ }^{13} \mathrm{C} \mathrm{NMR}\left(75 \mathrm{MHz}, \mathrm{CDCl}_{3}, \mathrm{TMS}\right) \delta$ 148.1, 131.0, 126.7, 124.3, 118.7, 117.8, 33.4, 24.5, 17.9; IR (KBr) v $2246.7 \mathrm{~cm}^{-1}$ (CN); MS (ESI) m/z (\%) 159.1(100) $\left[\mathrm{M}+\mathrm{H}^{+}\right]$, 181.1(22) $\left[\mathrm{M}+\mathrm{Na}^{+}\right]$; Anal. Calcd for $\mathrm{C}_{10} \mathrm{H}_{10} \mathrm{~N}_{2}$ : C, 75.92; H, 6.37; N, 17.71. Found: C, 75.99; H, 6.44; N, 17.45.

\section{Full characterization of all products}


S-(-)-1-(4-Fluorophenyl)aziridine-2-carboxamide (2b): white solid; mp 168-169 ${ }^{\circ} \mathrm{C} ;[\alpha]^{25}-170^{\circ}$ (c 1.0, $\mathrm{CHCl}_{3}$ ); ee $>99.5 \%$ (chiral HPLC analysis); ${ }^{1} \mathrm{H}$ NMR (300MHz, $\left.\mathrm{CDCl}_{3}, \mathrm{TMS}\right) \delta$ 6.87-6.99 (m, 4H), 6.48 (br, 1H), 6.17 (br, 1H), 2.68 (dd, $J$ $=3.0 \mathrm{~Hz}, 7.0 \mathrm{~Hz}, 1 \mathrm{H}), 2.47$ (dd, $J=1.1 \mathrm{~Hz}, 3.0 \mathrm{~Hz}, 1 \mathrm{H}), 2.32$ (dd, $J=1.1 \mathrm{~Hz}, 7.0 \mathrm{~Hz}$, 1H); ${ }^{13} \mathrm{C}$ NMR (75MHz, $\mathrm{CDCl}_{3}$, TMS) $\delta 172.2,160.2,157.0,147.3,147.2,121.2$, 121.1, 115.5, 115.2, 38.8, 34.4; $\quad$ IR $\quad(\mathrm{KBr}) \quad v \quad 3352.6, \quad 3167.5\left(\mathrm{CONH}_{2}\right)$, 1655.6 $\mathrm{cm}^{-1}(\mathrm{C}=\mathrm{O})$; MS (ESI) $\mathrm{m} / \mathrm{z}(\%) 181.2(100)\left[\mathrm{M}+\mathrm{H}^{+}\right]$, 203.1(71) $\left[\mathrm{M}+\mathrm{Na}^{+}\right]$. Anal. Calcd for $\mathrm{C}_{9} \mathrm{H}_{9} \mathrm{FN}_{2} \mathrm{O}$ : C, 59.99; H, 5.03; N, 15.55. Found: C, 60.03; H, 5.09; N, 15.48 . Methyl $\boldsymbol{R}$-(+)-1-(4-fluorophenyl)aziridine-2-carboxylate (4b): oil; $[\alpha]^{25}+83^{0}(C$ 0.75, $\mathrm{CH}_{2} \mathrm{Cl}_{2}$ ); ee 93.8\% (Chiral HPLC analysis) [lit. ${ }^{3}$ S-enantiomer: $[\alpha]^{25}-18.4^{\circ}(c$ 1.5, $\mathrm{CHCl}_{3}$ ); ee 7\%]; ${ }^{1} \mathrm{H} \mathrm{NMR}\left(300 \mathrm{MHz}, \mathrm{CDCl}_{3}, \mathrm{TMS}\right) \delta$ 6.93-6.96 (m, 4H), 3.81 (s, 3H), 2.77 (dd, $J=3.1 \mathrm{~Hz}, 6.4 \mathrm{~Hz}, 1 \mathrm{H}) 2.66$ (dd, $J=1.5 \mathrm{~Hz}, 3.1 \mathrm{~Hz}, 1 \mathrm{H}), 2.29$ (dd, $J=$ $1.5 \mathrm{~Hz}, 6.4 \mathrm{~Hz}, 1 \mathrm{H})$; IR (KBr) v 1750.1,(C=O).

S-(-)-1-(4-Chlorophenyl)aziridine-2-carboxamide (2c): white solid; mp 192-193 ${ }^{\circ} \mathrm{C} ;[\alpha]^{25}{ }_{\mathrm{D}}-158^{\circ}$ (c 1.0, $\mathrm{CHCl}_{3}$ ); ee 95.4\% (chiral HPLC analysis); ${ }^{1} \mathrm{H}$ NMR (300MHz, $\left.\mathrm{CDCl}_{3}, \mathrm{TMS}\right) \delta 7.21$ (d, $\left.J=9.6 \mathrm{~Hz}, 2 \mathrm{H}\right), 6.94$ (d, $\left.J=9.6 \mathrm{~Hz}, 2 \mathrm{H}\right), 6.41$ (br, 1H), 5.50 (br, 1H), 2.69 (dd, $J=3.1 \mathrm{~Hz}, 7.0 \mathrm{~Hz}, 1 \mathrm{H}), 2.49$ (dd, $J=1.2 \mathrm{~Hz}, 3.1 \mathrm{~Hz}, 1 \mathrm{H}$ ), 2.33 (dd, $J=1.2 \mathrm{~Hz}, 7.0 \mathrm{~Hz}, 1 \mathrm{H}) ;{ }^{13} \mathrm{C} \mathrm{NMR}\left(75 \mathrm{MHz}, \mathrm{CDCl}_{3}, \mathrm{TMS}\right) \delta 171.8,149.8,128.8$, 128.3, 121.3, 38.7, 34.3; IR (KBr) v 3401.8, 3242.7, $3193.5\left(\mathrm{CONH}_{2}\right), 1637.3 \mathrm{~cm}^{-1}$ (C=O); MS (ESI) m/z (\%)197.1(47) $\left[\mathrm{M}+\mathrm{H}^{+}\right], 219.0(100)\left[\mathrm{M}+\mathrm{Na}^{+}\right]$. Anal. Calcd for $\mathrm{C}_{9} \mathrm{H}_{9} \mathrm{ClN}_{2} \mathrm{O}$ : C, 54.97; H, 4.61; N, 14.25. Found: C, 54.96; H, 4.72; N, 13.99. 
Methyl $R$-(+)-1-(4-chlorophenyl)aziridine-2-carboxylate (4c): oil (lit. ${ }^{2}$ oil); $[\alpha]^{25}+140^{\circ}$ (c 1.3, $\mathrm{CH}_{2} \mathrm{Cl}_{2}$ ); ee 87.0\% (Chiral HPLC analysis); ${ }^{1} \mathrm{H}$ NMR (300M Hz, $\left.\mathrm{CDCl}_{3}, \mathrm{TMS}\right) \delta 7.21(\mathrm{~d}, J=6.7 \mathrm{~Hz}, 2 \mathrm{H}), 6.94(\mathrm{~d}, J=6.7 \mathrm{~Hz}, 2 \mathrm{H}), 3.81(\mathrm{~s}, 3 \mathrm{H}), 2.78$ (dd, 1H, $J=3.1 \mathrm{~Hz}, 6.3 \mathrm{~Hz}) 2.67$ (dd, $J=1.5 \mathrm{~Hz}, 3.1 \mathrm{~Hz}, 1 \mathrm{H}$ ), 2.29 (dd, $J=1.5 \mathrm{~Hz}$, $6.3 \mathrm{~Hz}, 1 \mathrm{H})$; IR $(\mathrm{KBr}) \vee 1748.2(\mathrm{C}=\mathrm{O}) \mathrm{cm}^{-1}$.

S-(-)-1-(4-Bromophenyl)aziridine-2-carboxamide (2d): white solid; mp 198-199 ${ }^{\circ} \mathrm{C} ;[\alpha]^{25}{ }_{\mathrm{D}}-162^{\circ}$ (c 1.0, $\mathrm{CHCl}_{3}$ ); ee 89.0\% (chiral HPLC analysis); ${ }^{1} \mathrm{H}$ NMR $(300 \mathrm{MHz}$, $\left.\mathrm{CDCl}_{3}, \mathrm{TMS}\right) \delta 7.35$ (d, $\left.J=9.6 \mathrm{~Hz}, 2 \mathrm{H}\right), 6.90$ (d, $\left.J=9.6 \mathrm{~Hz}, 2 \mathrm{H}\right), 6.42$ (br, 1H), 5.61 (br, 1H), 3.37 (dd, $J=3.0 \mathrm{~Hz}, 7.0 \mathrm{~Hz}, 1 \mathrm{H}$ ), 2.50 (dd, $J=1.2 \mathrm{~Hz}, 3.0 \mathrm{~Hz}, 1 \mathrm{H}$ ), 2.35 (dd, $J=1.2 \mathrm{~Hz}, 7.0 \mathrm{~Hz}, 1 \mathrm{H}) ;{ }^{13} \mathrm{C}$ NMR $\left(75 \mathrm{M} \mathrm{Hz}, \mathrm{CDCl}_{3}, \mathrm{TMS}\right) \delta 171.8,150.3,131.7$, 121.8, 115.8, 38.6, 34.2; IR (KBr) v 3428.8, 3362.3, $3203.2\left(\mathrm{CONH}_{2}\right), 1640.2 \mathrm{~cm}^{-1}$ (C=O); MS (ESI) m/z (\%)240.8(100) $\left[\mathrm{M}+\mathrm{H}^{+}\right]$, 242.8(100) $\left[\mathrm{M}+2+\mathrm{H}^{+}\right]$. Anal. Calcd for $\mathrm{C}_{9} \mathrm{H}_{9} \mathrm{BrN}_{2} \mathrm{O}$ : C, 44.84; H, 3.76; N, 11.62. Found: C, 44.88; H, 3.55; N, 11.40.

Methyl $R$-(+)- 1-(4-bromophenyl)aziridine-2-carboxylate (4d): white solid; mp 90-91 ${ }^{\circ} \mathrm{C} ;[\alpha]^{25}{ }_{\mathrm{D}}+157^{\circ}$ (c $0.65, \mathrm{CH}_{2} \mathrm{Cl}_{2}$ ); ee $87.0 \%$ (Chiral HPLC analysis) [lit. ${ }^{3}$ S-enantiomer: $[\alpha]^{25}-156.3^{\circ}$ (c $0.25, \mathrm{CHCl}_{3}$ ); ee 99\%]; ${ }^{1} \mathrm{H}$ NMR $\left(300 \mathrm{MHz}, \mathrm{CDCl}_{3}\right.$, TMS) $\delta 7.35$ (d, $J=6.7 \mathrm{~Hz}, 2 \mathrm{H}), 6.89$ (d, $J=6.7 \mathrm{~Hz}, 2 \mathrm{H}), 3.80$ (s, 3H), 2.78 (dd ,1H, $J=3.1 \mathrm{~Hz}, 6.3 \mathrm{~Hz}$ ), 2.67 (dd, $J=1.5 \mathrm{~Hz}, 3.1 \mathrm{~Hz}, 1 \mathrm{H}$ ), 2.29 (dd, $J=1.5 \mathrm{~Hz}, 6.3 \mathrm{~Hz}$, 1H); IR (KBr) v $1744.3 \mathrm{~cm}^{-1}(\mathrm{C}=\mathrm{O})$.

S-(-)-1-(4-Methoxyphenyl)aziridine-2-carboxamide (2e): white solid; mp 163-164 ${ }^{\circ} \mathrm{C} ;[\alpha]^{25}{ }_{\mathrm{D}}-184^{0}$ (c 1.0, $\mathrm{CHCl}_{3}$ ); ee $>99.5 \%$ (chiral HPLC analysis); ${ }^{1} \mathrm{H}$ NMR (300MHz, $\left.\mathrm{CDCl}_{3}, \mathrm{TMS}\right) \delta 6.92(\mathrm{~d}, J=8.8 \mathrm{~Hz}, 2 \mathrm{H}), 6.81$ (d, $\left.J=8.8 \mathrm{~Hz}, 2 \mathrm{H}\right), 6.49$ (br, 
1H), 5.66 (br, 1H), 3.77(s, 3H), 2.66 (dd, $J=2.2 \mathrm{~Hz}, 6.6 \mathrm{~Hz}, 1 \mathrm{H}), 2.43$ (br, 1H), 2.30 (d, $J=7.0 \mathrm{~Hz}, 1 \mathrm{H}) ;{ }^{13} \mathrm{C}$ NMR (75MHz, $\left.\mathrm{CDCl}_{3}, \mathrm{TMS}\right) \delta 172.4,155.4,144.5,120.8$, 114.0, 55.1, 38.8, 34.5; IR (KBr) v 3362.3, $3182.9\left(\mathrm{CONH}_{2}\right), 1661.4 \mathrm{~cm}^{-1}(\mathrm{C}=\mathrm{O})$; MS (ESI) $m / z(\%) 193.1(100)\left[\mathrm{M}+\mathrm{H}^{+}\right], 215.0$ (25) $\left[\mathrm{M}+\mathrm{Na}^{+}\right]$. Anal. Calcd for $\mathrm{C}_{10} \mathrm{H}_{12} \mathrm{~N}_{2} \mathrm{O}_{2}$ : C, 62.49; H, 6.29; N, 14.57. Found: C, 62.51; H, 6.30; N, 14.55.

Methyl $R$-(+)-1-(4-methoxyphenyl)aziridine-2-carboxylate (4e): white solid; mp 47-48 ${ }^{\circ} \mathrm{C} ;[\alpha]_{\mathrm{D}}^{25}+125^{\circ}$ (c $\left.0.4, \mathrm{CH}_{2} \mathrm{Cl}_{2}\right)$; ee $96.0 \%$ (Chiral HPLC analysis) [lit. ${ }^{3}$ S-enantiomer: $[\alpha]^{25}-184.3^{\circ}$ (c $0.5, \mathrm{CHCl}_{3}$ ); ee $79 \%$ ]; ${ }^{1} \mathrm{H}$ NMR $\left(300 \mathrm{MHz}, \mathrm{CDCl}_{3}\right.$, TMS) $\delta 6.95$ (d, $J=9.0 \mathrm{~Hz}, 2 \mathrm{H}), 6.79$ (d, $J=9.0 \mathrm{~Hz}, 2 \mathrm{H}), 3.80$ (s, 3H), 3.76 (s, 3H), 2.73 (dd, $J=3.3 \mathrm{~Hz}, 4.8 \mathrm{~Hz}, 1 \mathrm{H}), 2.63$ (s, 1H), 2.26 (d, $J=4.8 \mathrm{~Hz}, 1 \mathrm{H})$; IR (KBr) v $1744.3 \mathrm{~cm}^{-1}(\mathrm{C}=\mathrm{O})$. When biotransformation was quenched in $0.67 \mathrm{~h}, \mathrm{~S}-(-)-2 \mathbf{e}(28 \%$, ee $96.0 \%)$ and $R-(+)-4 e(22 \%$, ee $>99.5 \%)$ were obtained, along with the recovery of optically inactive nitrile 1 e (49\%, ee $0 \%)$.

S-(-)-1-p-Tolylaziridine-2-carboxamide (2f): white solid; mp 168-169 ${ }^{\circ} \mathrm{C} ;[\alpha]^{25}{ }_{\mathrm{D}}$ $-196^{\circ}$ (c 1.0, $\mathrm{CHCl}_{3}$ ); ee >99.5\% (chiral HPLC analysis); ${ }^{1} \mathrm{H}$ NMR $\left(300 \mathrm{MHz}, \mathrm{CDCl}_{3}\right.$, TMS) $\delta 7.07$ (d, $J=8.2 \mathrm{~Hz}, 2 \mathrm{H}), 6.91$ (d, $J=8.2 \mathrm{~Hz}, 2 \mathrm{H}), 6.57$ (br, 1H), 6.51 (br, 1H), 2.67 (dd, $J=3.0 \mathrm{~Hz}, 6.0 \mathrm{~Hz}, 1 \mathrm{H}$ ), 2.45 (dd, $J=1.2 \mathrm{~Hz}, 3.0 \mathrm{~Hz}, 1 \mathrm{H}), 2.32$ (dd, $J=1.2$ Hz, $6.0 \mathrm{~Hz}, 1 \mathrm{H}), 2.30$ (s, 3H); ${ }^{13} \mathrm{C}$ NMR (75M Hz, $\left.\mathrm{CDCl}_{3}, \mathrm{TMS}\right) \delta$ 173.3, 149.4, 133.1, 129.8, 120.4, 39.1, 24.7, 20.7; IR (KBr) v 3393.1, $3191.6\left(\mathrm{CONH}_{2}\right), 1631.5$ $\mathrm{cm}^{-1}(\mathrm{C}=\mathrm{O})$; MS (ESI) $\mathrm{m} / \mathrm{z}(\%) 177.2(100)\left[\mathrm{M}+\mathrm{H}^{+}\right]$. Anal. Calcd for $\mathrm{C}_{10} \mathrm{H}_{12} \mathrm{~N}_{2} \mathrm{O}: \mathrm{C}$, 68.16; H, 6.86; N, 15.90. Found: C, 67.79; H, 6.84; N, 15.90. 


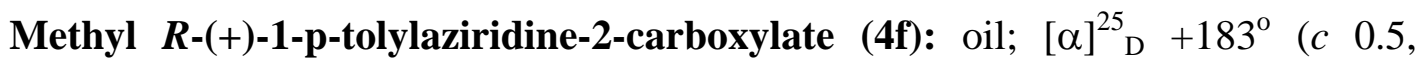
$\mathrm{CH}_{2} \mathrm{Cl}_{2}$ ); ee $>99.5 \%$ (Chiral HPLC analysis) $\left[\right.$ lit. $^{3}$ S-enantiomer: $[\alpha]^{25}{ }_{\mathrm{D}}-195.5^{\circ}(c$ 0.25, $\mathrm{CHCl}_{3}$ ); ee 70\%]; ${ }^{1} \mathrm{H} \mathrm{NMR}\left(300 \mathrm{MHz}, \mathrm{CDCl}_{3}, \mathrm{TMS}\right) \delta 7.04$ (d, $\left.J=6.5 \mathrm{~Hz}, 2 \mathrm{H}\right), 6.90$ (d, $J=6.5 \mathrm{~Hz}, 2 \mathrm{H}), 3.81$ (s, 3H), 2.75 (dd, $J=3.0 \mathrm{~Hz}, 6.2 \mathrm{~Hz}, 1 \mathrm{H}$ ), 2.64 (dd, $J=1.8$ $\mathrm{Hz}, 3.0 \mathrm{~Hz}, 1 \mathrm{H}), 2.27$ (dd, $J=1.8 \mathrm{~Hz}, 6.2 \mathrm{~Hz}, 1 \mathrm{H}) 2.28(\mathrm{~s}, 3 \mathrm{H})$; IR $(\mathrm{KBr}) v$ $1748.2 \mathrm{~cm}^{-1}(\mathrm{C}=\mathrm{O})$.

S-(-)-1-m-Tolylaziridine-2-carboxamide (2g): white solid; mp $179-180^{\circ} \mathrm{C}$; $[\alpha]^{25}$ $-162^{\circ}$ (c 1.0, $\mathrm{CHCl}_{3}$ ); ee 96.7\% (chiral HPLC analysis); ${ }^{1} \mathrm{H} \mathrm{NMR}\left(300 \mathrm{MHz}, \mathrm{CDCl}_{3}\right.$, TMS) $\delta 7.15$ (m, 1H), 6.78-6.87 (m, 3H), 6.46 (br, 1H), 5.52 (br, 1H), 2.70 (ddd, $J=$ 0.7 Hz, 3.0 Hz, 7.0 Hz, 1H), 2.45 (dd, $J=1.3 \mathrm{~Hz}, 3.0 \mathrm{~Hz}, 1 \mathrm{H}$ ), 2.35 (dd, $J=1.3 \mathrm{~Hz}$, $7.0 \mathrm{~Hz}, 1 \mathrm{H}), 2.32$ (s, 3H); ${ }^{13} \mathrm{C}$ NMR (75MHz, $\left.\mathrm{CDCl}_{3}, \mathrm{TMS}\right) \delta 173.1,151.7,139.2$, 129.1, 124.4, 121.3, 117.5, 39.0, 24.6, 21.3; IR (KBr) v 3355.5, $3186.8\left(\mathrm{CONH}_{2}\right)$, $1636.3 \mathrm{~cm}^{-1}(\mathrm{C}=\mathrm{O})$; MS (EI) m/z (\%) 176 (100) [M+1, 158 (55), 147 (54), 130 (65). Anal. Calcd for $\mathrm{C}_{10} \mathrm{H}_{12} \mathrm{~N}_{2} \mathrm{O}$ : C, 68.16; H, 6.86; N, 15.90. Found: C, 68.09; H, 6.89; N, 15.98.

Methyl $\boldsymbol{R}$-(+)-1-m-tolylaziridine-2-carboxylate (4g): oil; $[\alpha]_{\mathrm{D}}^{25}+104^{\circ}\left(\begin{array}{cc}c & 0.9\end{array}\right.$ $\mathrm{CH}_{2} \mathrm{Cl}_{2}$ ); ee $90.4 \%$ (Chiral HPLC analysis) [lit. ${ }^{4}$ S-enantiomer: $[\alpha]^{20}{ }_{\mathrm{D}}-43^{\circ}$ (c 0.45 , $\mathrm{CH}_{2} \mathrm{Cl}_{2}$ ); ee 20\%]; ${ }^{1} \mathrm{H}$ NMR (300MHz, $\mathrm{CDCl}_{3}$, TMS) $\delta$ 7.10-7.15 (m, 1H), 6.80-6.84 (m, 3H), 3.81 (s, 3H), 2.78 (dd, $J=3.0 \mathrm{~Hz}, 5.2 \mathrm{~Hz}, 1 \mathrm{H}$ ), 2.65 (dd, $J=1.8 \mathrm{~Hz}, 3.0 \mathrm{~Hz}$, 1H), 2.31 (s, 3H), 2.30 (dd, $J=1.8 \mathrm{~Hz}, 5.2 \mathrm{~Hz}, 1 \mathrm{H})$; IR (KBr) $v 1750.1 \mathrm{~cm}^{-1}(\mathrm{C}=\mathrm{O})$.

( \pm )-1-o-Tolylaziridine-2-carboxamide (2h): white solid; mp 163-164 ${ }^{\circ} \mathrm{C}$; $[\alpha]^{25}{ }_{\mathrm{D}} 0^{\circ}$; ee $0 \%$ (chiral HPLC analysis); ${ }^{1} \mathrm{H}$ NMR (300MHz, $\left.\mathrm{CDCl}_{3}, \mathrm{TMS}\right) \delta$ 7.11-7.23 (m, 2H), 
6.97-7.02 (m, 1H), 6.90 (d, $J=7.7 \mathrm{~Hz}, 1 \mathrm{H}), 6.51$ (br, 1H), 5.41 (br, 1H), 2.64 (ddd, $J$ $=0.9 \mathrm{~Hz}, 3.1 \mathrm{~Hz}, 6.9 \mathrm{~Hz}, 1 \mathrm{H}), 2.51$ (dd, $J=1.2 \mathrm{~Hz}, 3.1 \mathrm{~Hz}, 1 \mathrm{H}), 2.38$ (dd, $J=1.2 \mathrm{~Hz}$, $6.9 \mathrm{~Hz}, 1 \mathrm{H}) 2.32(\mathrm{~s}, 3 \mathrm{H}) ;{ }^{13} \mathrm{C} \mathrm{NMR}\left(75 \mathrm{MHz}, \mathrm{CDCl}_{3}, \mathrm{TMS}\right) \delta 173.1,149.2,130.8$, 130.6, 126.7, 123.6, 119.0, 39.2, 34.9, 18.0; IR (KBr) v $3336.33196 .4\left(\mathrm{CONH}_{2}\right)$, $1671.0 \mathrm{~cm}^{-1}(\mathrm{C}=\mathrm{O})$; MS (EI) m/z (\%) 176 (26) $\left[\mathrm{M}^{+}\right], 158$ (34), 132 (100), 118 (47), 117 (38). Anal. Calcd for $\mathrm{C}_{10} \mathrm{H}_{12} \mathrm{~N}_{2} \mathrm{O}$ : C, 68.16; H, 6.86; N, 15.90. Found: C, 67.97; H, 6.87; N, 15.84 .

Methyl ( \pm )-1-o-tolylaziridine-2-carboxylate (4h): oil; $[\alpha]^{25}{ }_{D}^{\circ}$; ee $0 \%$ (Chiral HPLC analysis); ${ }^{1} \mathrm{H}$ NMR (300M Hz, $\mathrm{CDCl}_{3}$, TMS) $\delta$ 7.08-7.13 (m, 2H), 6.96-6.98 (m, 1H), 6.83 (d, J = 7.9 Hz, 1H), 3.82 (s, 3H), 2.70 (dd, J = 4.0 Hz, 8.0Hz, 1H), 2.69 (s, 1H), 2.34 (dd, J = 4.0 Hz, 8.0 Hz, 1H), 2.32 (s, 3H); ${ }^{13} \mathrm{C}$ NMR (75MHz, $\mathrm{CDCl}_{3}$, TMS) $\delta 170.7,149.8,130.6,126.5,123.4,118.9,52.5,37.9,33.8,17.8$; IR (KBr) $v$ $1749.1 \mathrm{~cm}^{-1}(\mathrm{C}=\mathrm{O}) ; \mathrm{MS}(\mathrm{ESI}) \mathrm{m} / \mathrm{z}(\%) 192(100)\left[\mathrm{M}+\mathrm{H}^{+}\right]$. Anal. Calcd for $\mathrm{C}_{11} \mathrm{H}_{13} \mathrm{NO}_{2}$ : C, 69.09; H, 6.85; N, 7.32. Found: C, 69.08; H, 6.85; N, 7.60.

\section{R-(+)-2-[Benzyl(methyl)amino]-3-[benzyl(phenyl)amino]propanamide (15b):}

oil; $[\alpha]^{25}{ }_{\mathrm{D}}+10.0^{\circ}$ (c $1.4 \mathrm{CH}_{2} \mathrm{Cl}_{2}$ ); ee $91.0 \%$ (Chiral HPLC analysis); ${ }^{1} \mathrm{H}$ NMR (300MHz, $\left.\mathrm{CDCl}_{3}, \mathrm{TMS}\right) \delta$ 7.13-7.29 (m,12H), 6.69-6.77 (m, 3H), 6.38 (br, 1H), 6.01 (br, 1H), 4.68 (d, $J=17.0 \mathrm{~Hz}, 1 \mathrm{H}), 4.60$ (d, $J=17.0 \mathrm{~Hz}, 1 \mathrm{H}), 4.08$ (dd, $J=6.1 \mathrm{~Hz}$, 14.2 Hz, 1H), 3.60-3.79 (m, 4H), 2.34 (s, 3H); ${ }^{13} \mathrm{C}$ NMR (75MHz, $\left.\mathrm{CDCl}_{3}, \mathrm{TMS}\right) \delta$ 174.4, 147.9, 138.9, 138.7, 129.4, 128.7, 128.6, 128.5, 127.4, 127.0, 126.8, 117.2, 113.7, 62.7, 59.5, 55.7, 49.5, 38.3; IR (KBr) v 3451.0, 3327.6, $3185.8\left(\mathrm{CONH}_{2}\right)$, 
$1681.6 \mathrm{~cm}^{-1}(\mathrm{C}=\mathrm{O})$; MS (ESI) $\mathrm{m} / \mathrm{z}(\%) 374(100)\left[\mathrm{M}+\mathrm{H}^{+}\right]$. Anal. Calcd for $\mathrm{C}_{24} \mathrm{H}_{27} \mathrm{~N}_{3} \mathrm{O}$ : C, 77.18; H, 7.29; N, 11.25. Found: C, 77.48; H, 7.05; N, 11.20.

$\boldsymbol{R}$-(-)-2-Allylamino-3-[benzyl(phenyl)amino]propanamide $\quad(15 c): \quad$ oil; $\quad[\alpha]^{25}$ $-25.4^{\circ}$ (c $3.6 \mathrm{CH}_{2} \mathrm{Cl}_{2}$ ) ee 84\% (Chiral HPLC analysis); ${ }^{1} \mathrm{H}$ NMR (300MHz, $\mathrm{CDCl}_{3}$, TMS) $\delta$ 7.16-7.31(m, 7H), 7.04 (br,1H), 6.74-6.89 (m, 3H), 6.15 (br, 1H), 5.64-5.77 (m, 1H), 5.00-5.09 (m, 2H), 4.57 (d, $J=17.5 \mathrm{~Hz}, 1 \mathrm{H}), 4.55$ (d, $J=17.5 \mathrm{~Hz}, 1 \mathrm{H}$ ), 3.78 (dt, $J=9.7 \mathrm{~Hz}, 1 \mathrm{H}), 3.46-3.53$ (m, 2H), 3.15 (dd, $J=5.3 \mathrm{~Hz}, 14.2 \mathrm{~Hz}, 1 \mathrm{H}$ ), 2.96 (dd, $J$ $=6.4 \mathrm{~Hz}, 14.2 \mathrm{~Hz}, 1 \mathrm{H}) ;{ }^{13} \mathrm{C}$ NMR $\left(75 \mathrm{MHz}, \mathrm{CDCl}_{3}, \mathrm{TMS}\right) \delta 176.2,148.8,138.3$, 135.7, 129.4, 128.7, 127.1, 126.9, 118.2, 116.4, 114.1, 61.2, 56.0, 54.9, 51.1; IR (KBr) v 3431.7, $3321.8\left(\mathrm{CONH}_{2}\right), 3207.7(\mathrm{~N}-\mathrm{H}), 2245.7\left(\mathrm{CH}_{2}=\mathrm{CH}\right), 1681.6 \mathrm{~cm}^{-1}(\mathrm{C}=\mathrm{O}) ; \mathrm{MS}$ (ESI) $\mathrm{m} / \mathrm{z}(\%) 310(100)\left[\mathrm{M}+\mathrm{H}^{+}\right], 332$ (8) $\left[\mathrm{M}+\mathrm{Na}^{+}\right]$Anal. Calcd for $\mathrm{C}_{19} \mathrm{H}_{23} \mathrm{~N}_{3} \mathrm{O}$ : 309.1841. Found: 309.1839.

R-(-)-2-Azido-3-[benzyl(phenyl)amino]propanamide (15d): oil; $[\alpha]^{25}{ }_{D}-36.0^{\circ}$ (c 1.1 $\mathrm{CH}_{2} \mathrm{Cl}_{2}$ ); ee $78.3 \%$ (Chiral HPLC analysis); ${ }^{1} \mathrm{H}$ NMR (300MHz, $\left.\mathrm{CDCl}_{3}, \mathrm{TMS}\right) \delta$ 7.17-7.32 (m, 7H), 6.75-6.88 (m, 3H), 6.37 (br,1H), 5.96 (br,1H), 4.77 (d, J = 17.0 Hz, 1H), 4.61 (d, J = 17.0 Hz, 1H), 4.40 (dd, J = 3.4 Hz, 9.6 Hz, 1H), 4.23 (dd, J = 3.4 $\mathrm{Hz}, 15.2 \mathrm{~Hz}, 1 \mathrm{H}), 3.52$ (dd, J = 9.6 Hz, $15.2 \mathrm{~Hz}, 1 \mathrm{H}) ;{ }^{13} \mathrm{C} \mathrm{NMR}\left(75 \mathrm{MHz}, \mathrm{CDCl}_{3}\right.$, TMS) $\delta 170.6,147.1,137.8,129.5,128.7,127.1,126.7,118.1,113.3,62.1,55.3,54.4$; IR $(\mathrm{KBr})$ v 3456.8, $3375.8\left(\mathrm{CONH}_{2}\right), 2103.0\left(\mathrm{~N}_{3}\right), 1676.8 \mathrm{~cm}^{-1}(\mathrm{C}=\mathrm{O})$; MS (ESI) m/z (\%) 296 (100) $\left[\mathrm{M}+\mathrm{H}^{+}\right]$. Anal. Calcd for $\mathrm{C}_{16} \mathrm{H}_{17} \mathrm{~N}_{5} \mathrm{O}$ : C, 65.07; H, 5.80; N, 23.71. Found: C, 65.28; H, 5.89; N, 23.52. 
4. ${ }^{1} \mathrm{H}$ and ${ }^{13} \mathrm{C}$ NMR spectra of $1 \mathrm{a}-1 \mathrm{~h}, 2 \mathrm{a}-2 \mathrm{~h}, 4 \mathrm{a}-4 \mathrm{~h}, 15 \mathrm{a}-\mathrm{d}, 17$. 


\section{1a- ${ }^{1} \mathrm{H}-\mathrm{NMR}$}
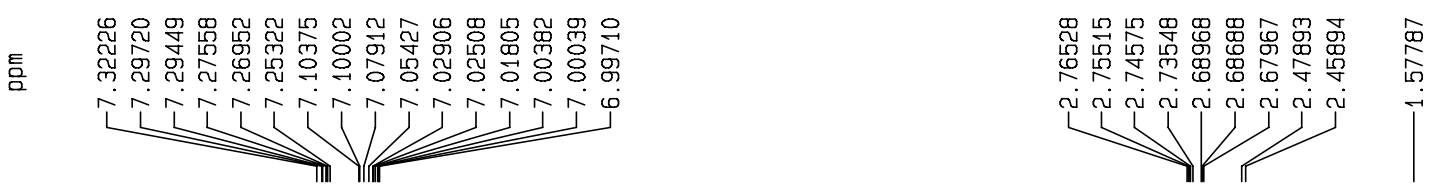

Current Data Parameters

NAME WJy05-0910a

PHOCNO

F2 - Acquisition Parameters

Jate_

INSTRUM

TD $\quad 65536$

JS

2
$=$ IDPES
$6172.839 \mathrm{~Hz}$

$\begin{array}{ll}0.094190 \mathrm{~Hz} \\ A Q & 5.3084660 \mathrm{sec}\end{array}$

$\begin{array}{lr}A Q & 5.3084660 \\ 7 G & 203 .\end{array}$

$\begin{array}{rr}\text { JW } & 203.2 \\ \text { JW } & 81.000 \text { usec } \\ \text { JE } & 6.00 \text { usec }\end{array}$

6.00 usec
$673.2 \mathrm{~K}$

$2.00000000 \mathrm{sec}$

$=======$ CHANNEL $f 11======$
VUC 1 H

$\begin{array}{ll}\text { NUC1 } & \text { IH } \\ 71 & 900 \text { us }\end{array}$

$-1.00 \mathrm{~dB}$
$\mathrm{LF}$

Theters

F2 - Processing parameters
SI 32768

$\begin{array}{lc}\text { SF } & 300.1300083 \mathrm{MHz} \\ \mathrm{SFW} & \mathrm{EM}\end{array}$

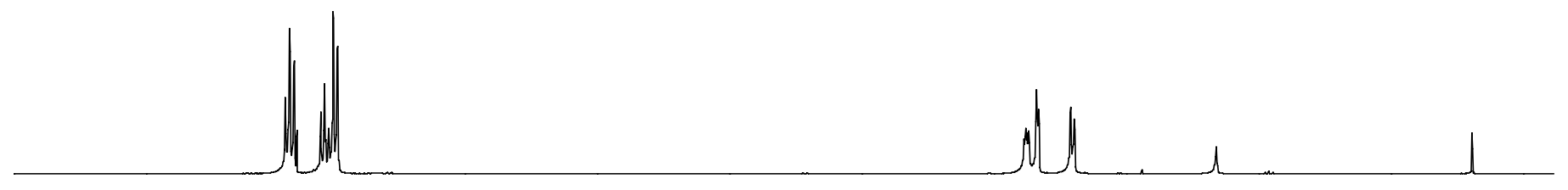

$\llcorner B$
GB
PC

$10 \mathrm{NMR}$ plot parameters
$\mathrm{CX}$
$22.00 \mathrm{~cm}$

$\begin{array}{lr}c X & 22.00 \mathrm{~cm} \\ c Y & 2.50 \mathrm{~cm}\end{array}$

$\begin{array}{lr}F 1 P & 9.000 \mathrm{ppm} \\ =1 & 2701.17 \mathrm{~Hz}\end{array}$

$\begin{array}{ll}2 \mathrm{P} & -0.500 \mathrm{ppm} \\ -2 & -0.0307 \mathrm{~Hz}\end{array}$

$\begin{array}{lr}\text { F2 } & -150.07 \mathrm{~Hz} \\ \text { PPMCM } & 0.43182 \mathrm{ppm} / \mathrm{cm} \\ \text { HZCM } & 129.60159 \mathrm{~Hz} / \mathrm{cm}\end{array}$
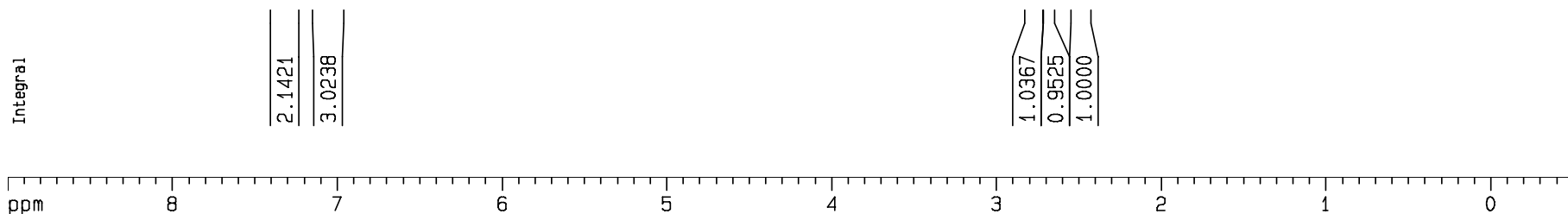
1b- ${ }^{1} \mathrm{H}-\mathrm{NMR}$

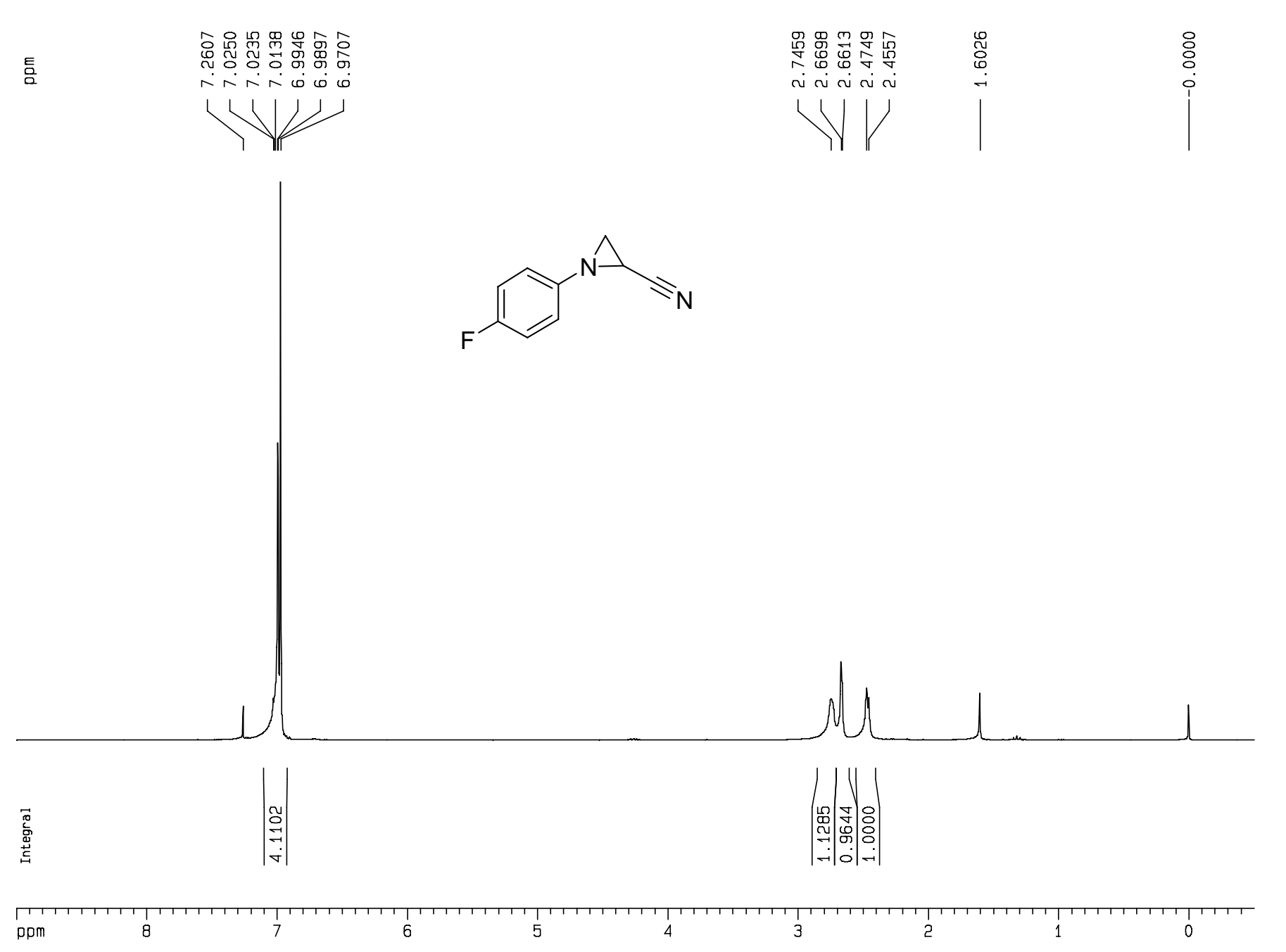




\section{1c- ${ }^{1} \mathrm{H}-\mathrm{NMR}$}
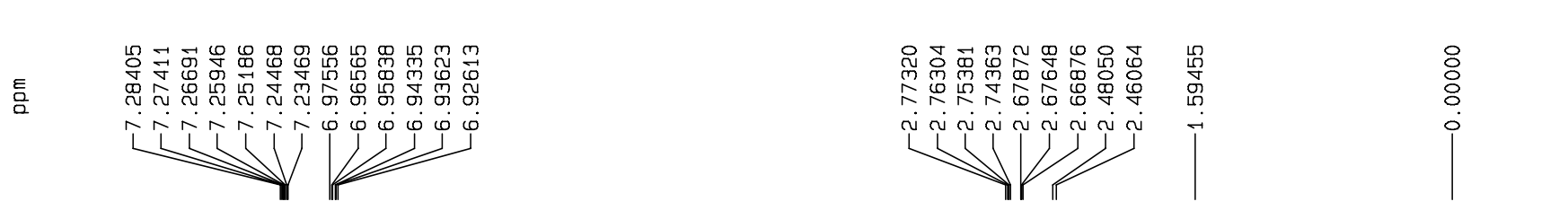

Current Data Parameters

NAME WJy05-0928C

PROCNO

F2 - Acquisition Parameter

$\begin{array}{ll}\text { Date } & 20050928 \\ \text { Time } & \end{array}$

$\begin{array}{ll}\text { Time } & 22.46 \\ \text { INSTRUM } & \text { av300 }\end{array}$

PROBHD $5 \mathrm{~mm}$ DUL 13C-1

$\begin{array}{lr}\text { PULPROG } & 2930 \\ \text { TD } & 65536\end{array}$

$\begin{array}{ll}\text { TD } & 65536 \\ \text { SOLVENT } & \text { CDC13 }\end{array}$
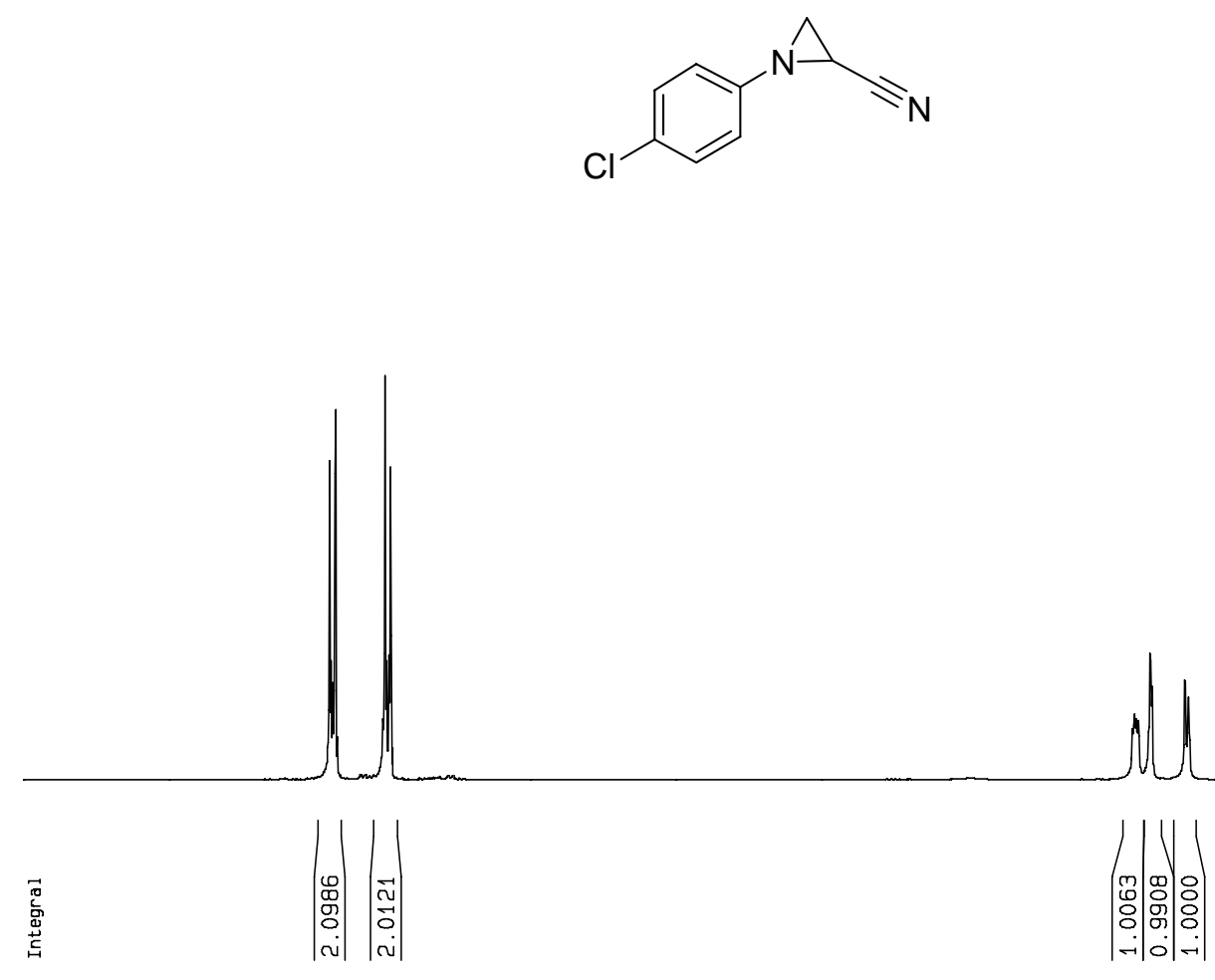

$\begin{array}{lr}\text { DS } & 2 \\ \text { SWH } & 6172.839 \mathrm{~Hz}\end{array}$

$0.094190 \mathrm{~Hz}$
FID
AQ

$\begin{array}{ll}\text { AQ } & 0.0948600 \mathrm{~Hz} \\ \mathrm{AG} & 203.2\end{array}$

203.2
81.000 usec

6.00 use

$298.9 \mathrm{~K}$

$=======$ CHANNEL $f 1=======$
NUC1

$\begin{array}{lr}\text { NUC1 } & 1 \mathrm{H} \\ \mathrm{P1} & 9.30 \mathrm{use}\end{array}$

$-1.00 \mathrm{~dB}$

SF01 300.1318534 MHZ

F2 - Processing parameters

$\begin{array}{lc}\text { SI } & 32768 \\ \text { SF } & 300.1300064 \mathrm{MHZ}\end{array}$

WOW $\quad 00$

$\begin{array}{lc}\text { SSB } & 0 \\ \text { LB } & 0.30 \mathrm{HZ} \\ G B & 0\end{array}$

10 NMF plot parameters

$\begin{array}{lr}\text { CX } & 22.00 \mathrm{~cm} \\ \text { CY } & 5.00 \mathrm{~cm} \\ \text { FIP } & 9.000 \mathrm{ppm} \\ \text { F1 } & 2701.17 \mathrm{~Hz} \\ \text { F2P } & -0.500 \mathrm{ppm} \\ \text { F2 } & -150.07 \mathrm{~Hz} \\ \text { PPMCM } & 0.43182 \mathrm{ppm} / \mathrm{cm} \\ \text { HZCM } & 129.60159 \mathrm{~Hz} / \mathrm{cm}\end{array}$




\section{1d- ${ }^{1} \mathrm{H}-\mathrm{NMR}$}

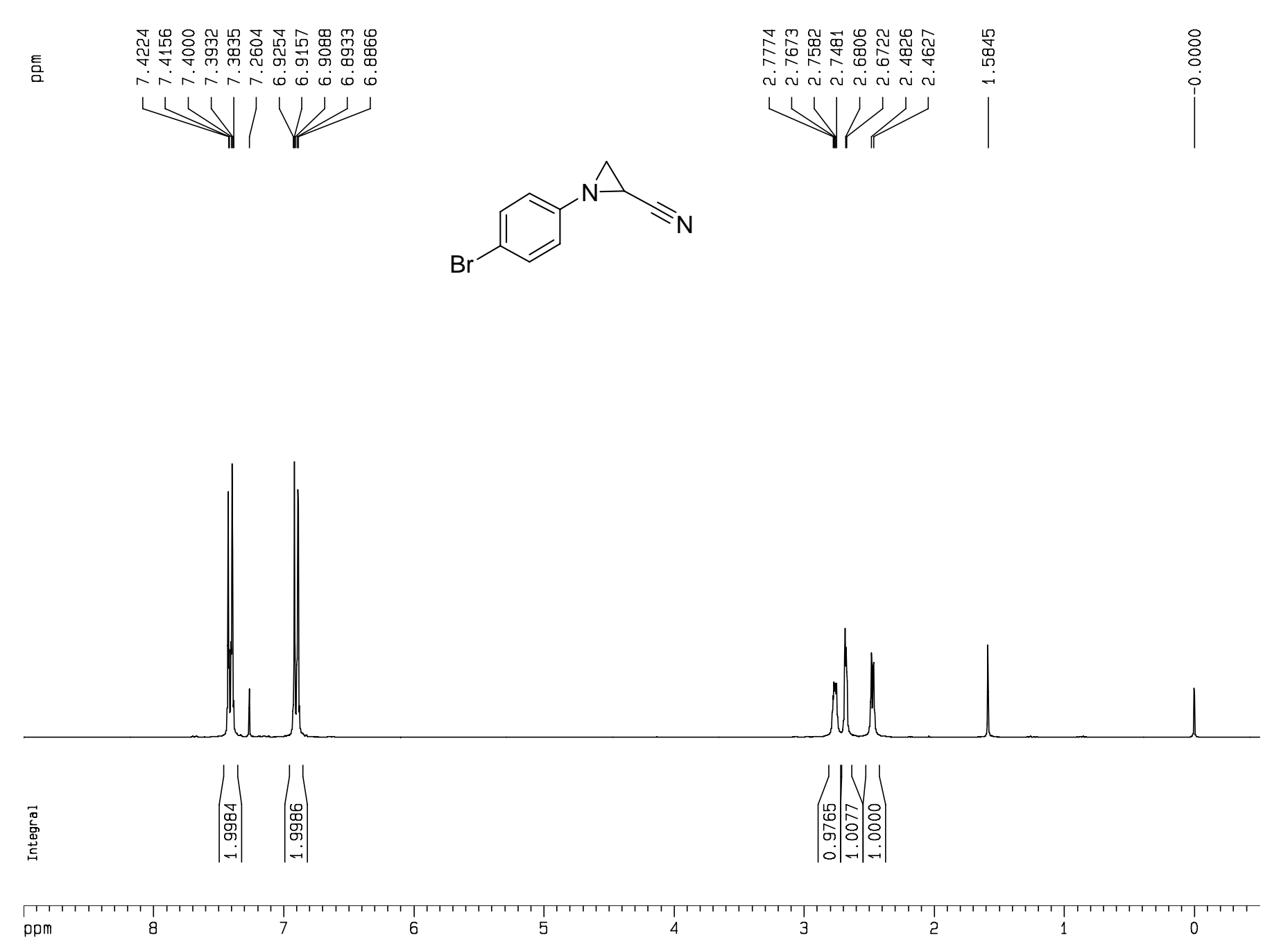

Current Data Parameters

NAME wjy05-1107a

EXPNO

F2 - Acquisition Parameter

Date_ $\quad 20051107$

INSTRUM av300

PROBHD $5 \mathrm{~mm}$ DUL 13C-

$\begin{array}{lr}\text { PULPROG } & 2930 \\ \text { TO } & 65536\end{array}$

SOLVENT CDC1

$\begin{array}{lr}\text { DS } & 2 \\ & 5172.839 \\ \end{array}$

$\begin{array}{ll}\text { SWH } & 6172.839 \mathrm{~Hz} \\ \text { FIDRES } & 0.094190 \mathrm{~Hz}\end{array}$

AQ $\quad 5.3084660 \mathrm{sec}$

$\begin{array}{lr}\text { RG } & 322.5 \\ \text { OW } & 81.000 \text { usec }\end{array}$

6.00 usec
$297.4 \mathrm{~K}$ $2.00000000 \mathrm{sec}$

$=======$ CHANNEL $f 1=======$
NUC1 $1 \mathrm{H}$

$\begin{array}{lr}\mathrm{P} 1 & 9.30 \text { use }\end{array}$

SF01 $300.1318534 \mathrm{MHz}$

F2 - Processing parameters

SI 32768

SF $\quad 300.1300062 \mathrm{MH}$

$\begin{array}{lc}\text { WOW } & \text { EM } \\ \text { SSB } & 0 \\ \text { LB } & 0.30 \mathrm{~Hz}\end{array}$

10 NMR plot parameters

$\begin{array}{lr}\text { CX } & 22.00 \mathrm{~cm} \\ \mathrm{CY} & 5.00 \mathrm{~cm}\end{array}$

$\begin{array}{lr}\text { CY } & 5.00 \mathrm{~cm} \\ \text { F1P } & 9.000 \mathrm{ppm}\end{array}$

F2P $\quad-0.500 \mathrm{ppm}$

F2 $\quad-150.07 \mathrm{~Hz}$

$\begin{array}{lr}\text { PPMCM } & 0.43182 \mathrm{ppm} / \mathrm{cm} \\ \text { HZCM } & 129.60159 \mathrm{~Hz} / \mathrm{cm}\end{array}$ 
1d- ${ }^{13} \mathrm{C}-\mathrm{NMR}$

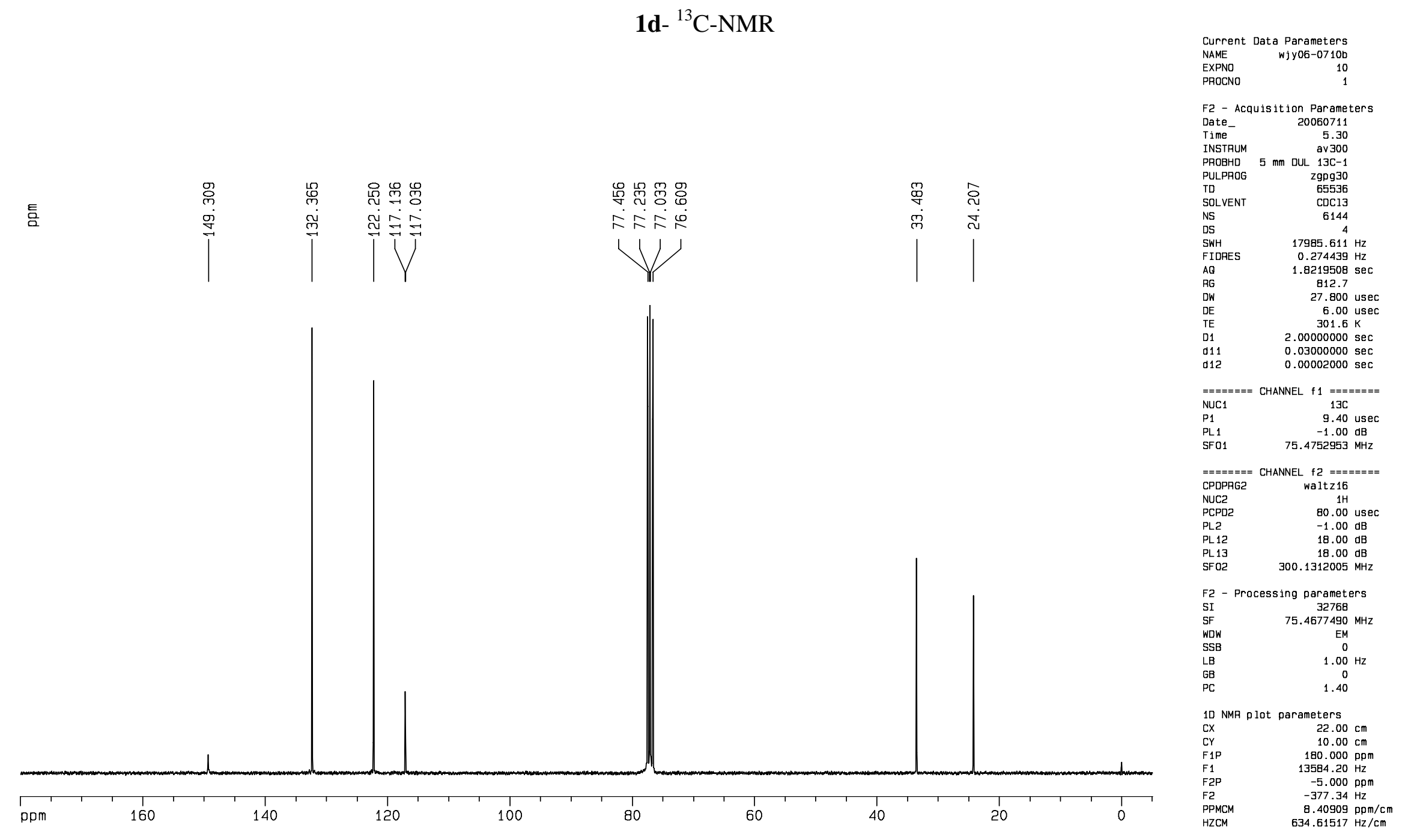




\section{1e- ${ }^{1} \mathrm{H}-\mathrm{NMR}$}

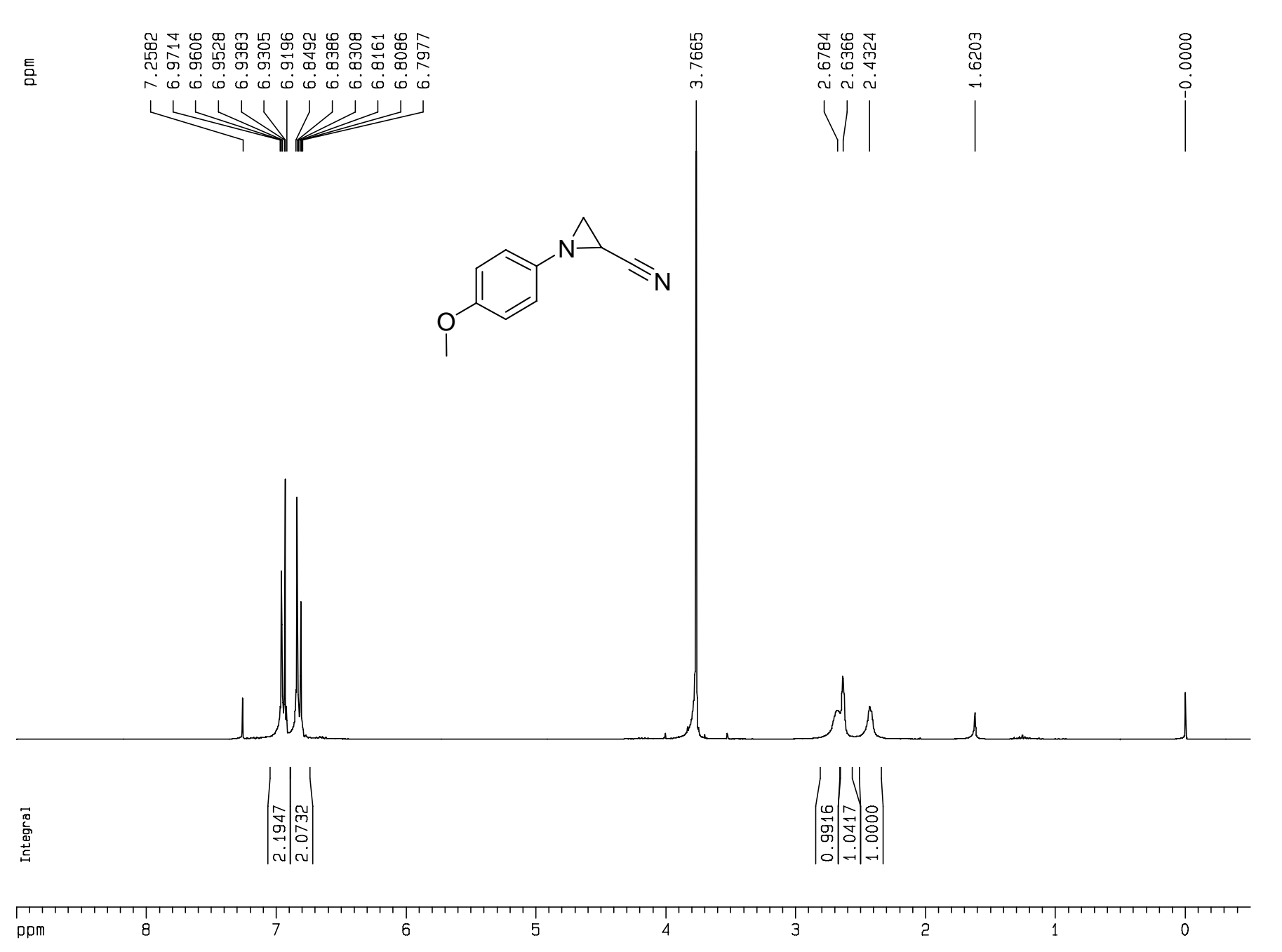




\section{1f- ${ }^{1} \mathrm{H}-\mathrm{NMR}$}

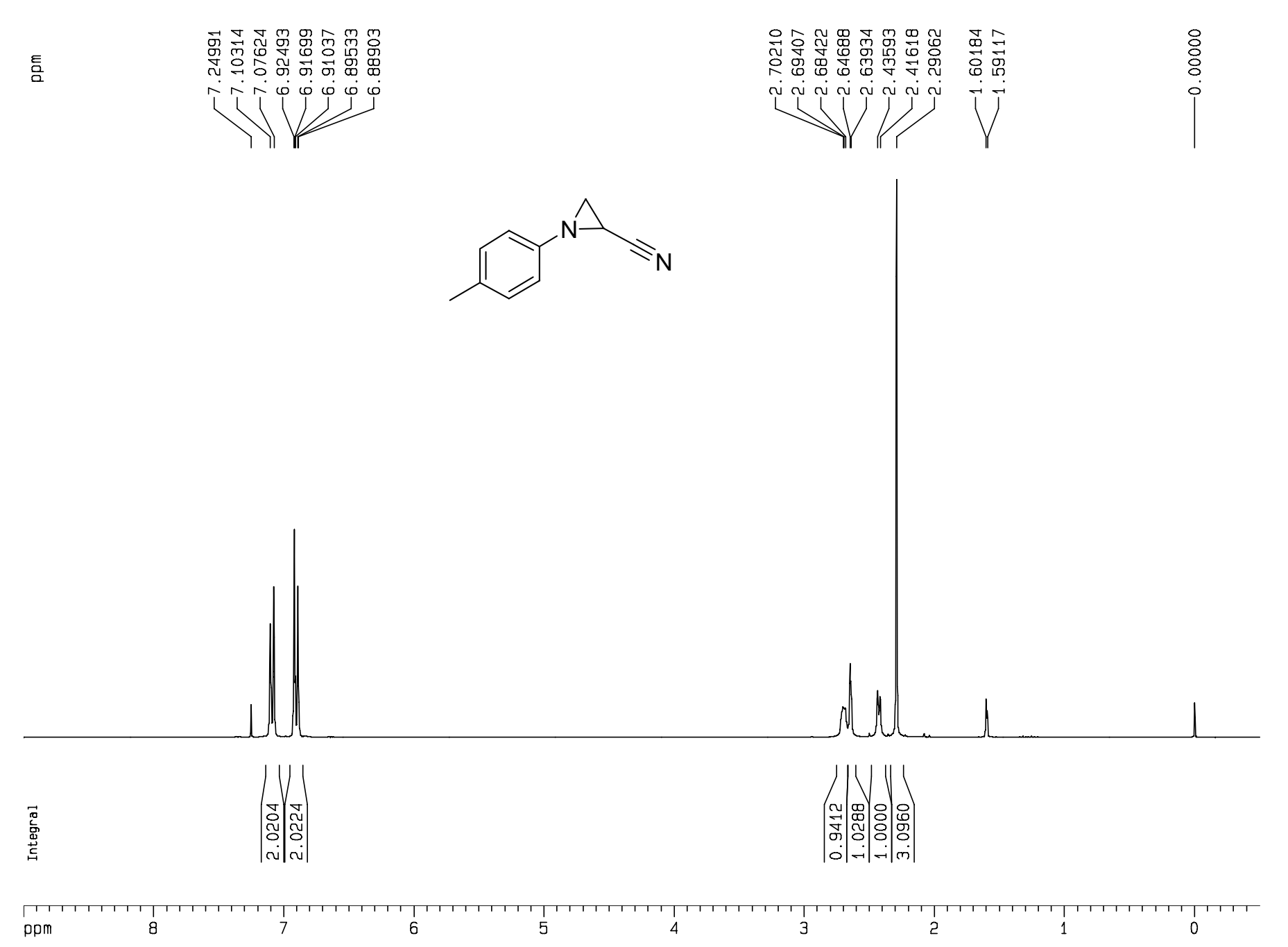

Current Data Parameters

NAME wjy05-0928b

EXPNO

F2 - Acquisition Parameters

Date

Time $\quad 14.27$

INSTRUM av300

PROBHD $5 \mathrm{~mm}$ DUL 13C-1

$\begin{array}{lr}\text { PULPROG } & 2930 \\ \text { TO } & 65536\end{array}$

SOLVENT $\quad$ CDC13

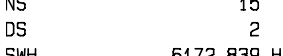

$\begin{array}{ll}\text { SWH } & 6172.839 \mathrm{~Hz} \\ \text { FIDRES } & 0.094190 \mathrm{~Hz}\end{array}$

AQ $\quad 5.3084660 \mathrm{sec}$

$\begin{array}{lr}\text { RG } & 181 \\ \text { DW } & 81.000 \text { usec }\end{array}$

81.000 usec
6.00 usec $2.00000000 \mathrm{sec}$

$=======$ CHANNEL $f 1=======$
NUC1

P1 9.30 use

SF01 $300.1318534 \mathrm{MHz}$

F2 - Processing parameters

SI 32768

SF $\quad 300.1300094 \mathrm{MHH}$

WDW

$\begin{array}{lc}\text { SSB } & 0 \\ \text { LB } & 0.30 \mathrm{~Hz} \\ \text { GB } & 0 \\ \text { PC } & 1.00\end{array}$

10 NMR plot parameters

$\begin{array}{ll}C X & 22.00 \mathrm{~cm} \\ C Y & 10.00 \mathrm{~cm}\end{array}$

$\begin{array}{lr}\text { CY } & 10.00 \mathrm{~cm} \\ \text { F1P } & 9.000 \mathrm{ppm}\end{array}$

F2P $\quad-0.500 \mathrm{ppm}$

F2 $\quad-150.07 \mathrm{~Hz}$

$\begin{array}{lr}\text { PPMCM } & 0.43182 \mathrm{ppm} / \mathrm{cm} \\ \text { HZCM } & 129.60159 \mathrm{~Hz} / \mathrm{cm}\end{array}$ 
1g- ${ }^{1} \mathrm{H}-\mathrm{NMR}$

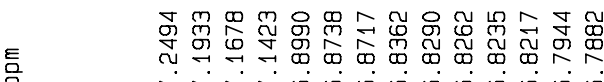

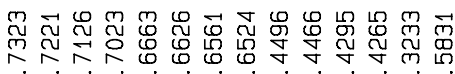

|

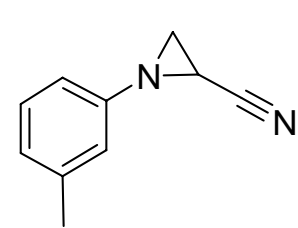

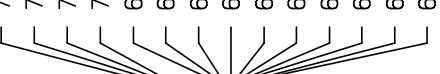

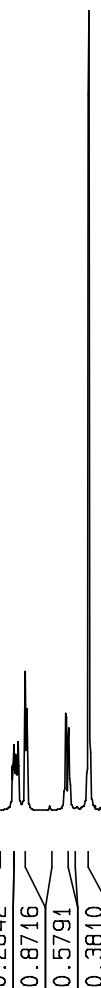

Current Data Parameters

NAME WJ W05-1018b

EXPNO

2 - Acquisition Parameter

Time

22.58

INSTRUM av300

PROBHD $5 \mathrm{~mm}$ DUL 13C-1

$\begin{array}{lr}\text { PULPROG } & 2930 \\ \text { TD } & 65536\end{array}$

SOLVENT COC13

$\begin{array}{ll}\text { NS } & 9 \\ \text { DS } & 5 \\ \text { FH } & 0.09490 \mathrm{~Hz}\end{array}$

$\begin{array}{ll}\text { SWH } & 6172.839 \mathrm{~Hz} \\ \text { FIDRES } & 0.094190 \mathrm{~Hz}\end{array}$

$A Q \quad 5.3084660 \mathrm{sec}$

$\begin{array}{lr}\text { RG } & 256 \\ \text { DW } & 81.000 \text { usec }\end{array}$

81.000 usec
6.00 usec $2.00000000 \mathrm{sec}$

$=======$ CHANNEL $f 1=======$
NUC1

$\begin{array}{ll}\text { NUC1 } & 1 \mathrm{H} \\ \mathrm{P} 1 & 9.30 \text { usec }\end{array}$

SF01 $300.1318534 \mathrm{MHz}$

F2 - Processing parameters

SI 32768

SF $\quad 300.1300096 \mathrm{MHZ}$

$\begin{array}{lc}\text { WDW } & \text { EM } \\ \text { SSB } & 0 \\ \text { L } & 0.30 \mathrm{~Hz}\end{array}$

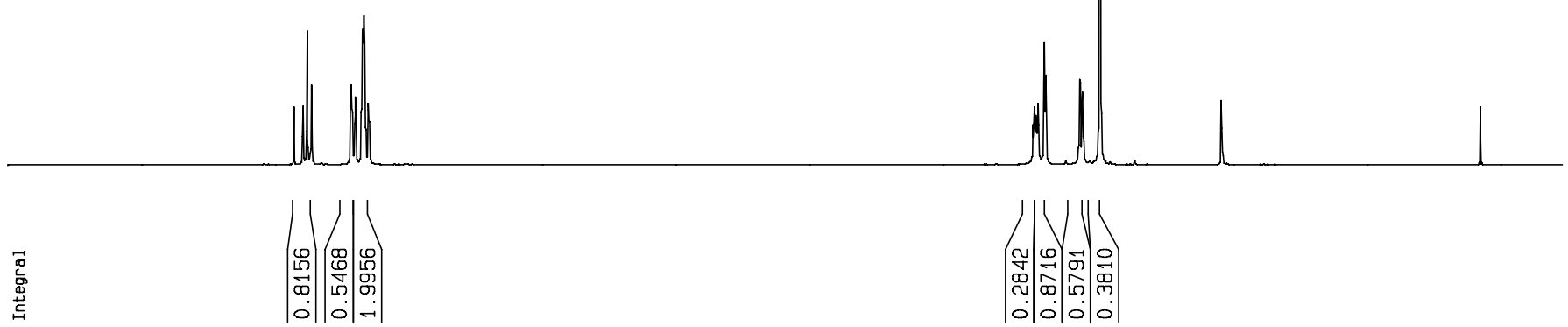

1.00

10 NMR plot parameters

$\begin{array}{ll}\text { CX } & 22.00 \mathrm{~cm} \\ \mathrm{CY} & 10.00 \mathrm{~cm}\end{array}$

CY $\quad 9.00 \mathrm{~cm}$

$\begin{array}{ll}F 1 & 2701.17 \mathrm{~Hz} \\ F 2 \mathrm{P} & -0.500 \mathrm{PP}\end{array}$

F2 $\quad-150.07 \mathrm{~Hz}$

PPMCM $\quad 0.43182 \mathrm{ppm} / \mathrm{cm}$

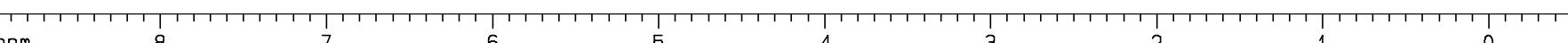

ppm 
1g- ${ }^{13} \mathrm{C}-\mathrm{NMR}$
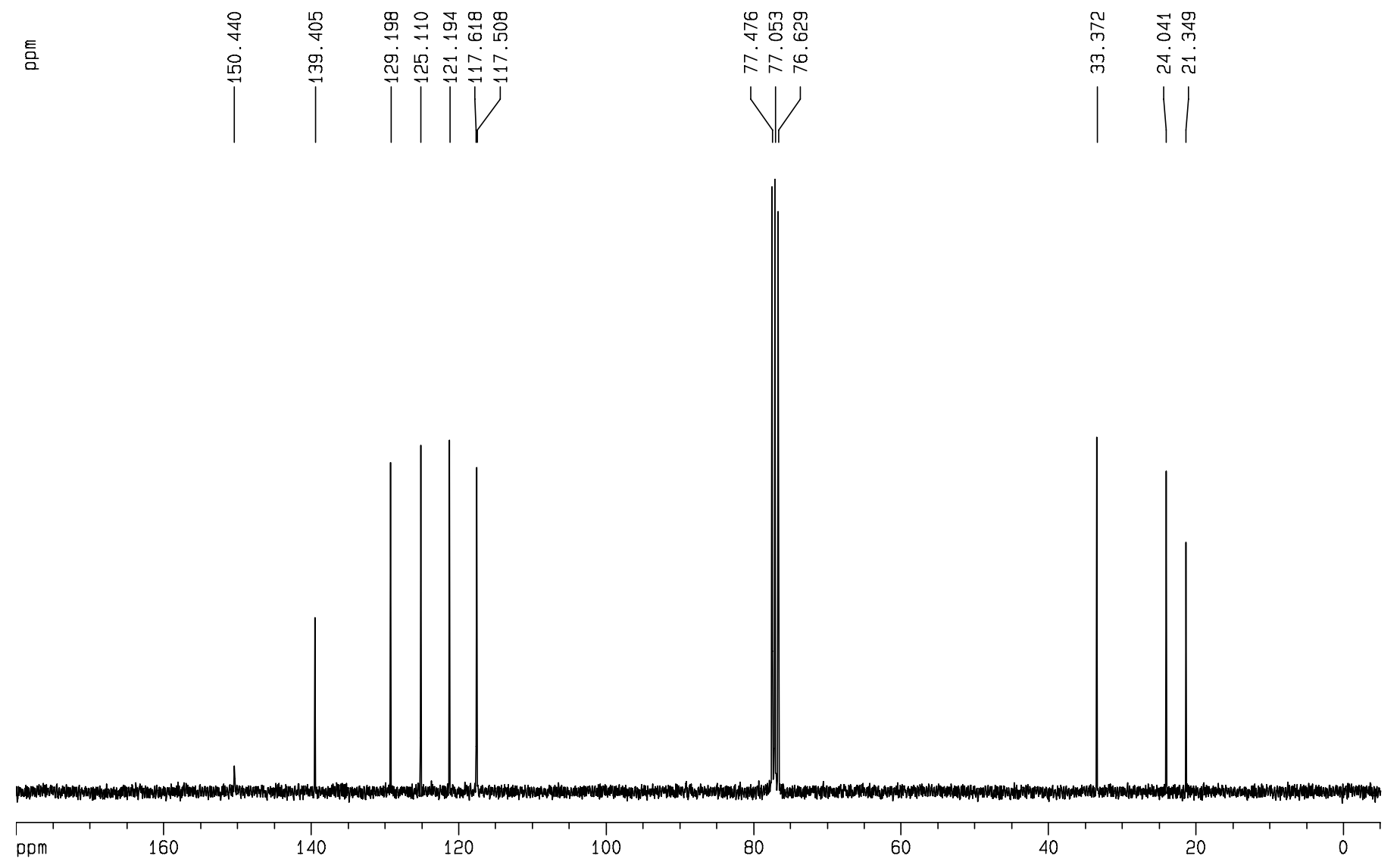

Current Data Parameters

PROCNO

F2 - Acquisition Parameters

Date
Time
Time

$\begin{array}{ll}\text { Time } & 21.57 \\ \text { INSTRUM } & \text { av300 }\end{array}$

PULPROG Zgpg30

$\begin{array}{ll}\text { TD } & 65536 \\ \text { SOLVENT } & \text { CDC13 }\end{array}$

4
$\mathrm{DS}$

SWH
FIDRES

$\begin{array}{ll}\text { AQ } & 1.8219508 \mathrm{sec} \\ \text { AG } & 4096\end{array}$

$\begin{array}{lr}\mathrm{BG} & 4096 \\ \mathrm{DW} & 27.800 \mathrm{usec} \\ \mathrm{DE} & 5.00 \mathrm{usec}\end{array}$

TE $300.3 \mathrm{~K}$

$\begin{array}{ll}01 & 2.00000000 \mathrm{sec} \\ \mathrm{d11} & 0.03000000 \mathrm{sec}\end{array}$

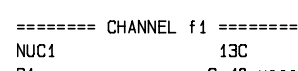

$\begin{array}{lr}\text { P1 } & 9.40 \text { usec } \\ \text { PL1 } & -1.00 \mathrm{~dB} \\ \text { SF01 } & 75.4752953 \mathrm{MHz}\end{array}$

$======$ CHANNEL $f 2=======$

$\begin{array}{lr}\text { CPDPPG } & \text { waltz16 } \\ \text { NUC2 } & 1 \mathrm{H} \\ \text { PCPD2 } & 80.00 \text {. }\end{array}$

$\begin{array}{ll}\text { NUC2 } & 1 \mathrm{H} \\ \text { PCD2 } & 8.00 \mathrm{usec} \\ \text { PL2 } & -1.00 \mathrm{~dB}\end{array}$

$\begin{array}{lr}\text { PL12 } & 18.00 \mathrm{~dB} \\ \text { PL13 } & 18.00 \mathrm{~dB} \\ \text { SF02 } & 300.1312005 \mathrm{MHZ}\end{array}$

F2 - Processing parameters

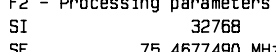

$\begin{array}{ll}\text { SF } & 75.4677490 \mathrm{MHZ} \\ \text { WOW } & \text { EM }\end{array}$

$\begin{array}{lc}\text { SSB } & 0 \\ L B & 1.00 \mathrm{~Hz} \\ G B & 0 \\ \text { PC } & 1.40\end{array}$

10 NMR p plot parameters

CX $\quad 22.00 \mathrm{~cm}$

$\begin{array}{lr}\mathrm{CY} & 10.00 \mathrm{~cm} \\ F 1 \mathrm{P} & 180.000 \mathrm{ppm} \\ =1 & 13584.20 \mathrm{~Hz}\end{array}$

$\begin{array}{lr}F 1 & 13584.20 \mathrm{~Hz} \\ F 2 \mathrm{P} & -5.000 \mathrm{ppm} \\ F 2 & -377.34 \mathrm{~Hz}\end{array}$

$\begin{array}{ll}\text { F2 } & -377.34 \mathrm{~Hz} \\ \text { PPMCM } & 8.40909 \mathrm{ppm} / \mathrm{cm}\end{array}$ 


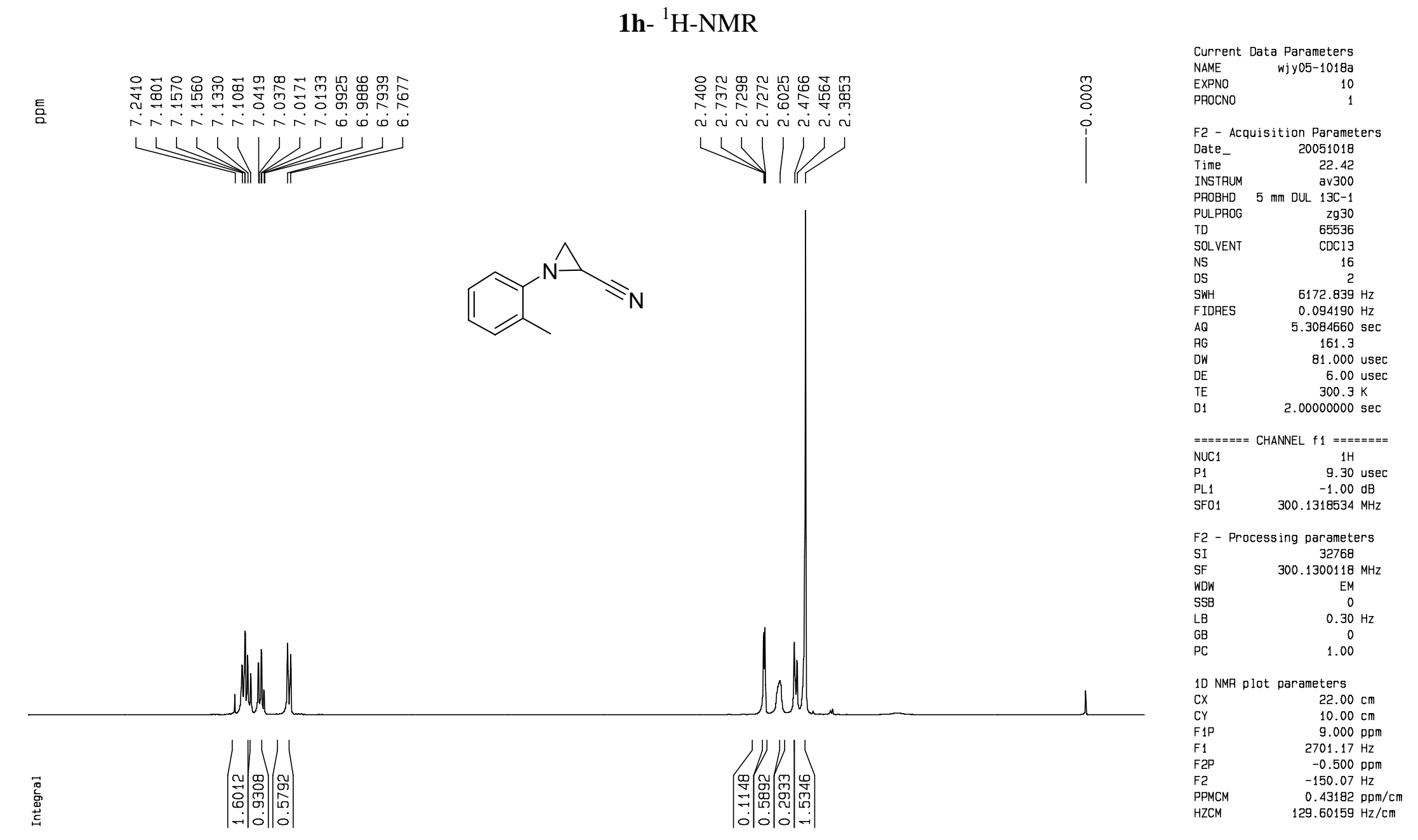


1h- ${ }^{13}$ C-NMR

言

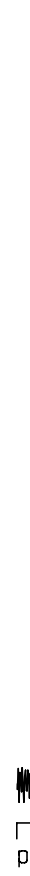

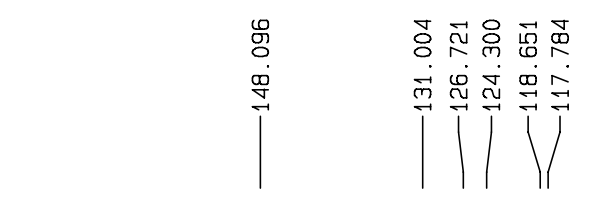

||||||||

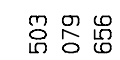

송

inj

160
80

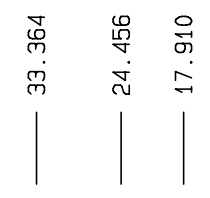

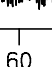

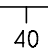

,
1
Current Data Parameters

NAME

F2 - Acquisition Parameters
Date 20051018

Date_-

22.46
INSTRUM

PROBHD $5 \mathrm{~mm}$ DUL 13C-1
PULPROG

$\begin{array}{lr}\text { PULPROG } & \text { zgpg30 } \\ \text { TD } & 65536 \\ \text { SOLVENT } & \text { CDC13 }\end{array}$

$\begin{array}{lr}\text { NS } & 105 \\ \text { JS } & 4\end{array}$

$\begin{array}{ll}\text { SWH } & 4 \\ \text { IDHRES } & 17985.611 \mathrm{~Hz}\end{array}$

$\begin{array}{ll}\mathrm{AQ} & 0.274439 \mathrm{HZ} \\ \mathrm{AQ} & 1.8219508 \mathrm{seC}\end{array}$

$\begin{array}{ll}\mathrm{AQ} & 1.8219508 \mathrm{sec} \\ \mathrm{AG} & 2298.8 \\ \mathrm{JW} & 27.800 \mathrm{usec}\end{array}$

$\begin{array}{ll} & \\ \mathrm{TE} & 6.00 \mathrm{use} \\ \mathrm{TE} & 300.5 \mathrm{~K}\end{array}$

d1

$\mathrm{d} 11$
$\mathrm{~d} 2$ $2.00000000 \mathrm{sec}$
$0.03000000 \mathrm{sec}$
$0.00002000 \mathrm{sec}$

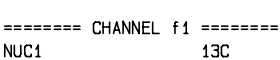

$\begin{array}{lr}\text { P.1 } & 9.40 \text { usec } \\ \text { PL1 } & -1.00 \mathrm{~dB}\end{array}$

PL1
SF01

$======$ CHANNEL $f 2===== \pm=$
CPDPAG2

$\begin{array}{lc}\text { CPDPAG2 } & \text { waltz16 } \\ \text { NUC2 } & 1 \mathrm{H} \\ \text { गCPO2 } & 80.00 \text { usec } \\ & \end{array}$

$\begin{array}{ll}80.00 \mathrm{US} \\ \text { PL2 } & -1.00 \mathrm{~dB}\end{array}$

$\begin{array}{rr}\text { PL12 } & 18.00 \mathrm{~dB} \\ \text { PL13 } & 18.00 \mathrm{~dB} \\ \text { SF } & 3002\end{array}$

F2 - Processing parameters

$\begin{array}{ll}\mathrm{SI} & 32768 \\ \mathrm{SF} & 75.4677490 \mathrm{MHZ}\end{array}$

$\begin{array}{lc}\text { SF } & 75.4677490 \mathrm{MHZ} \\ \text { WOW } & \text { EM } \\ \text { SSB } & 0\end{array}$

LB
GB
GC

10 NMR plot parameters

$\begin{array}{ll}\text { CX } & 22.00 \mathrm{~cm} \\ \mathrm{CY} & 10.00 \mathrm{~cm}\end{array}$

$\begin{array}{lr}\mathrm{CY} & 10.00 \mathrm{~cm} \\ =1 \mathrm{P} & 180.00 \mathrm{ppm} \\ =1 & 13584.20 \mathrm{~Hz}\end{array}$

$\begin{array}{ll}=2 \mathrm{P} & -5.000 \mathrm{ppm} \\ =2 & -377.34 \mathrm{~Hz}\end{array}$

$\begin{array}{lr}\text { F2 } & -377.34 \mathrm{~Hz} \\ \text { PPMCM } & 8.40909 \mathrm{p} \mathrm{pm} / \mathrm{cm} \\ \text { HZCM } & 634.61517 \mathrm{~Hz} / \mathrm{cm}\end{array}$ 
2a- ${ }^{1} \mathrm{H}-\mathrm{NMR}$

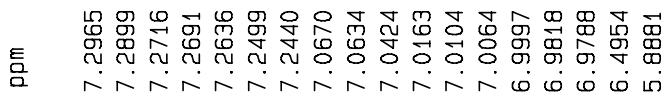
番)

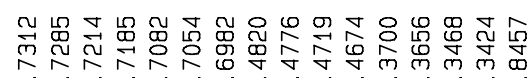

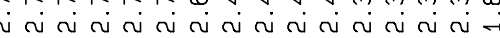

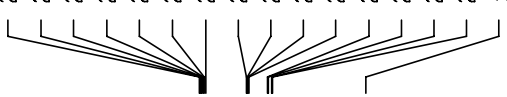<smiles>NC(=O)C1CN1c1ccccc1</smiles>

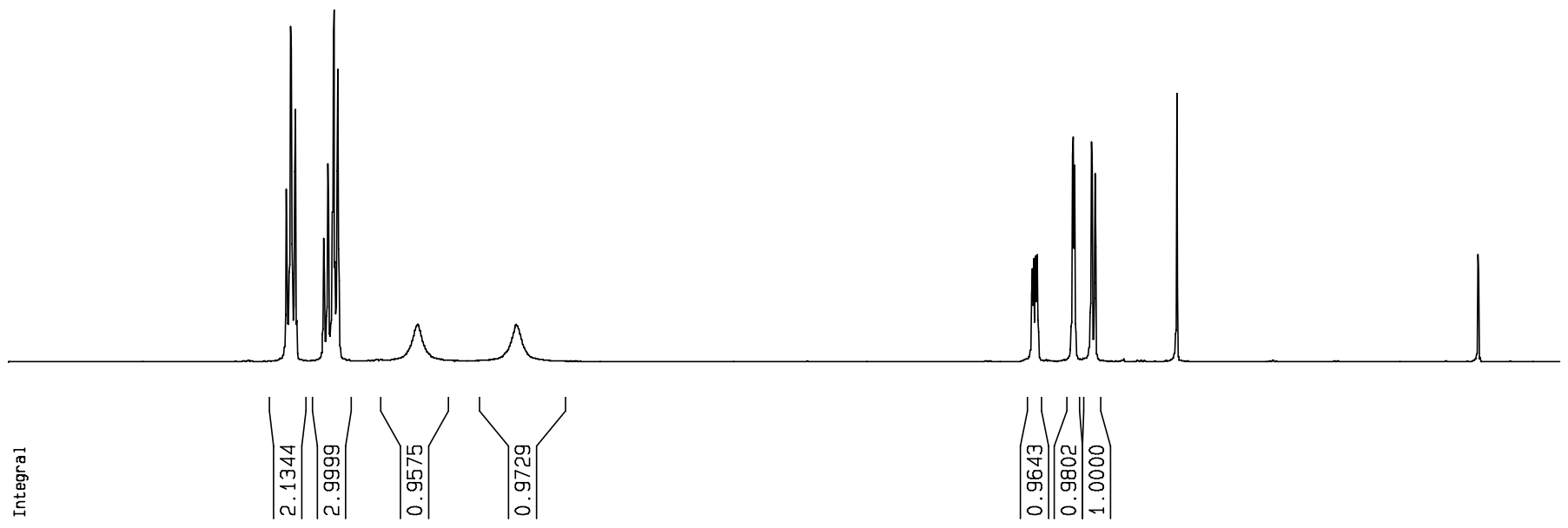

pp

ppn

6

5
4 
2b- ${ }^{1} \mathrm{H}-\mathrm{NMR}$

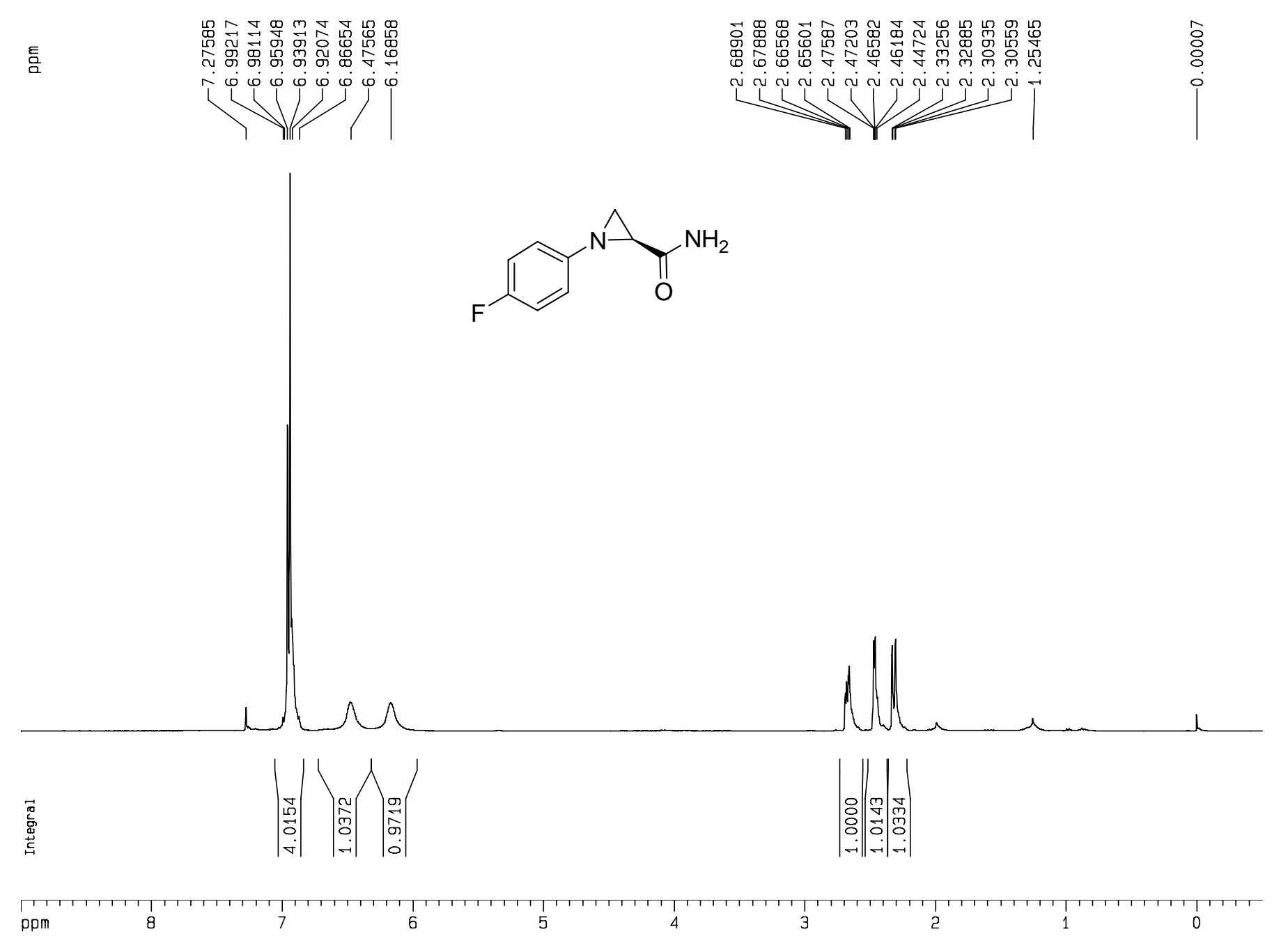

Current Data Parameters

NAME wjy05-1229a

EXPNO

F2 - Acquisition Parameter

Date $\quad 20051229$

$\begin{array}{ll}\text { Time } & 16.45 \\ \text { INSTRUM } & \text { av300 }\end{array}$

PROBHD $5 \mathrm{~mm}$ DUL $13 \mathrm{C}-1$

2930

$\begin{array}{ll}\text { TD } & 65536 \\ \text { SOLVENT } & \text { CDC13 }\end{array}$

NS 16

SWH $\quad 6172.839 \mathrm{~Hz}$

FIDRES $\quad 0.094190 \mathrm{~Hz}$

AQ $\quad 5.3084660 \mathrm{sec}$

$\begin{array}{lr}\text { RG } & 181 \\ \text { DW } & 81.000 \text { usec }\end{array}$

81.000 usec
6.00 usec
$295.3 \mathrm{~K}$

$2.00000000 \mathrm{sec}$

$=======$ CHANNEL $f 1=======$
NUC1 $1 \mathrm{H}$

P1 $\quad 11.20$ use

$\begin{array}{ll}\text { PFO1 } & 0.00 \mathrm{~dB} \\ & 300.1318534 \mathrm{MHz}\end{array}$

F2 - Processing parameters

SI 32768

WOW $\quad 300.1300018 \mathrm{MHZ}$

$\begin{array}{lc}\text { SSB } & \text { EM } \\ \text { LB } & 0 \\ & 0.30 \mathrm{~Hz}\end{array}$

10 NMR plot parameters

$\begin{array}{ll}\text { CX } & 22.00 \mathrm{~cm} \\ \text { CY } & \text { Fy }\end{array}$

$\begin{array}{lr}\text { CY } & 10.00 \mathrm{~cm} \\ \text { F1P } & 9.000 \mathrm{ppm}\end{array}$

$\begin{array}{ll}F 1 & 2701.17 \mathrm{~Hz} \\ \text { F2P } & -0.500 \mathrm{ppm}\end{array}$

F2 $\quad-150.07 \mathrm{~Hz}$

$\begin{array}{lr}\text { PPMCM } & 0.43182 \mathrm{ppm} / \mathrm{cm} \\ \text { HZCM } & 129.60159 \mathrm{~Hz} / \mathrm{cm}\end{array}$ 
2b- ${ }^{13}$ C-NMR

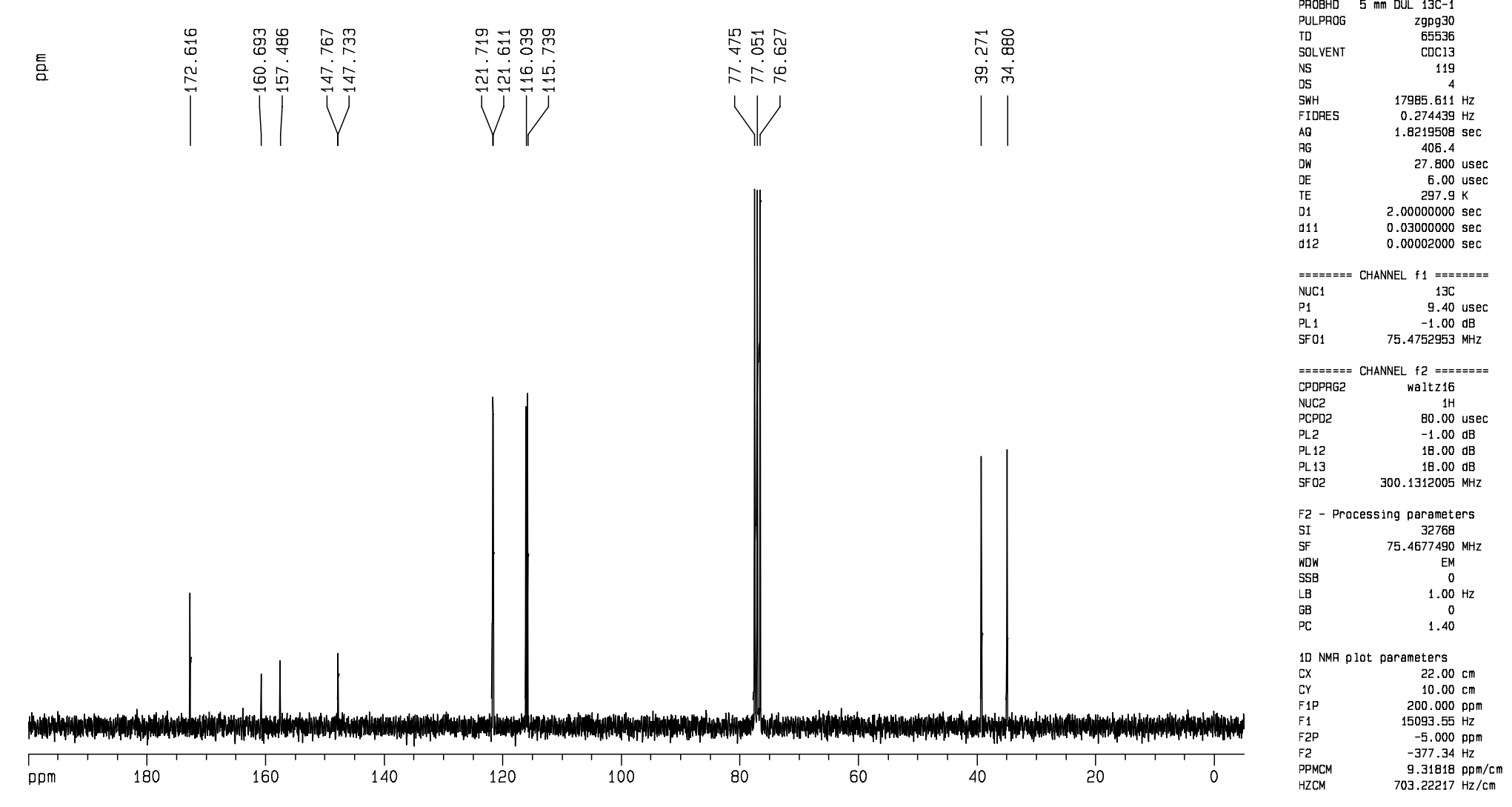




\section{2c- ${ }^{1} \mathrm{H}-\mathrm{NMR}$}
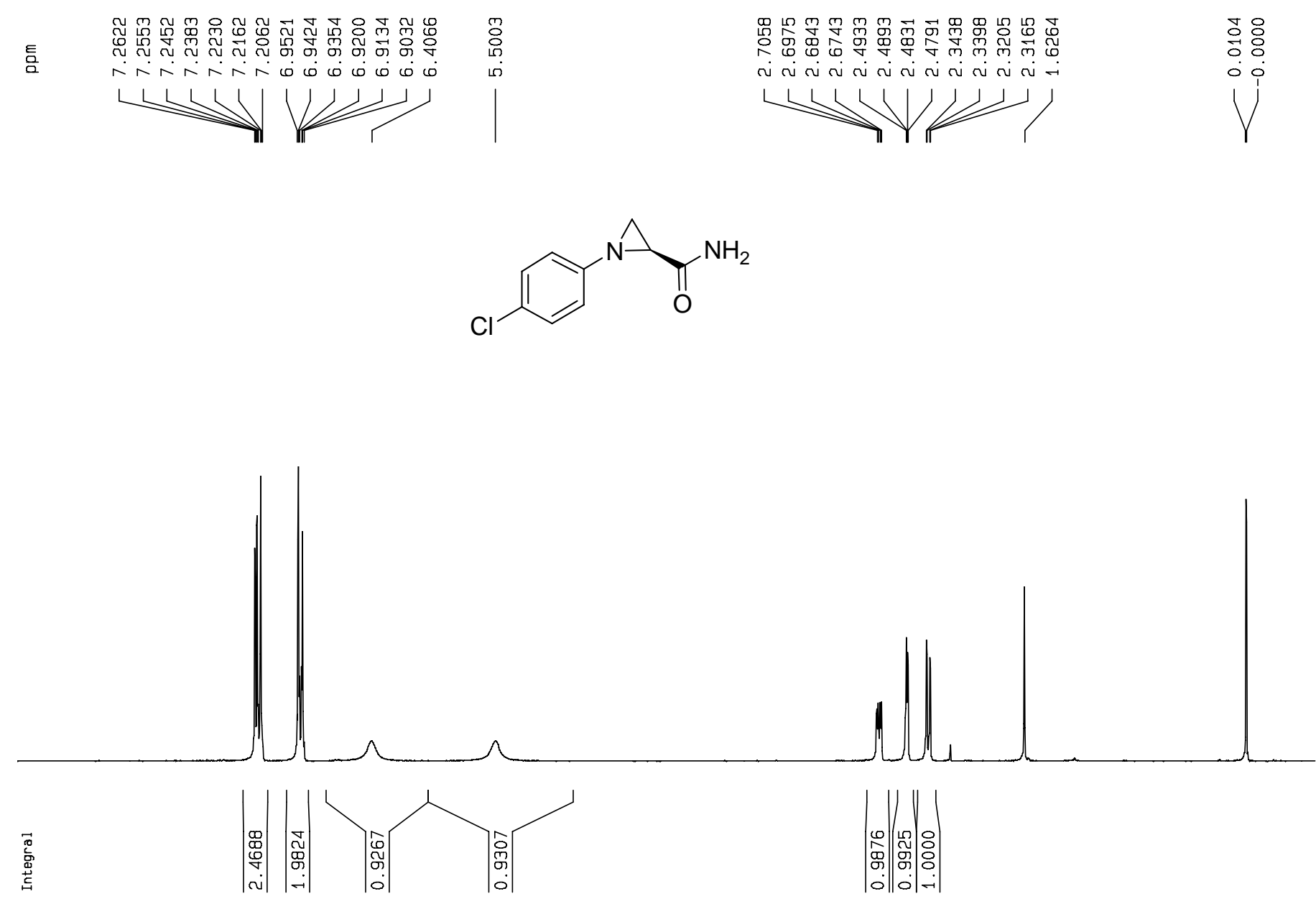

ppm
6

5
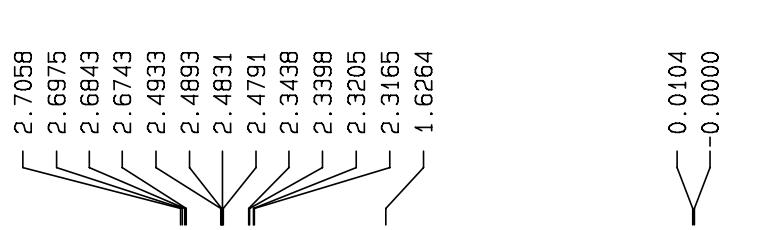

Current Data Parameters

NAME wjy06-0530a

EXPNO
PROCNO

F2 - Acquisition Parameters

Date
Time 20060530
16.38

INSTRUM av300 $5 \mathrm{~mm}$ DUL 13C-1 \begin{tabular}{lr} 
PULPROG & 2930 \\
TD & 65536 \\
\hline
\end{tabular} $\begin{array}{lr}\text { SOLVENT } & \text { CDC13 } \\ \text { NS } & 16 \\ \text { DS } & 5\end{array}$

$\begin{array}{lr}\text { JS } & 2 \\ \text { SWH } & 6172.839 \mathrm{~Hz}\end{array}$

IDRES $\quad 0.094190 \mathrm{~Hz}$

AQ $\quad 5.3084660 \mathrm{sec}$ $\begin{array}{lr}\text { AG } & 406.4 \\ \text { JW } & 81.000 \text { usec } \\ \text { JE } & 6.00 \text { usec }\end{array}$ $301.1 \mathrm{~K}$

D1 $2.00000000 \mathrm{sec}$

$===$

$\begin{array}{lr}\text { NUC1 } & 1 \mathrm{H} \\ \mathrm{P}_{1} & 9.30 \text { usec }\end{array}$

$\begin{array}{lr}\text { PL1 } & -1.00 \mathrm{~dB} \\ \text { SF01 } & 300.1318534\end{array}$

F2 - Processing parameters
SI
S2768

$\begin{array}{lc}\text { SF } & 300.1300056 \mathrm{MHz} \\ \text { WDW } & \text { EM }\end{array}$

SSB
$\llcorner B$
GB

${ }_{P C}$

10 NMA plot parameters
CX $22.00 \mathrm{~cm}$

$\begin{array}{lr}\mathrm{CY} & 5.00 \mathrm{~cm} \\ =1 \mathrm{P} & 9.000 \mathrm{~cm}\end{array}$

$\begin{array}{lr}=1 \mathrm{P} & 9.000 \mathrm{ppm} \\ =1 & 2701.17 \mathrm{~Hz}\end{array}$

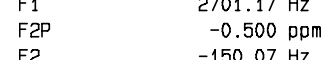

गPMCM $\quad-150.07 \mathrm{~Hz}$

$\begin{array}{ll} & \\ \text { HZCM } & 129.60159 \mathrm{~Hz} / \mathrm{cm}\end{array}$ 
2c- ${ }^{13} \mathrm{C}-\mathrm{NMR}$

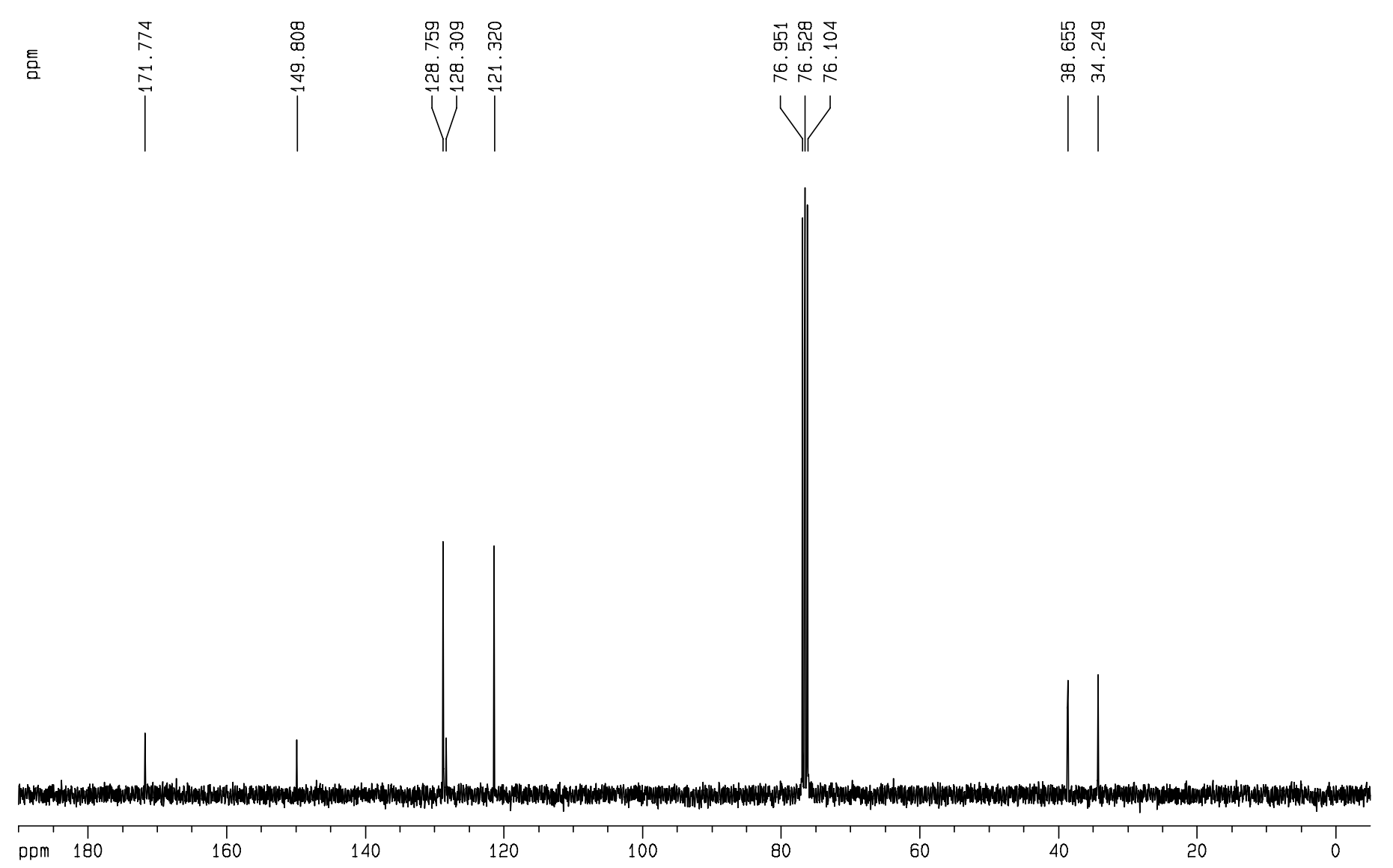

Current Data Parameters

EXPNO WJY05-1229g

PROCNO

F2 - Acquisition Parameters

Date
Time

PROBHD $5 \mathrm{~mm}$ DUL $13 \mathrm{a}$ 1300

PULPROG ZgPg30

\begin{tabular}{lr} 
TD & 65536 \\
SOLVENT & COC13 \\
NS & 139 \\
\hline
\end{tabular}

4

$\begin{array}{lr}\text { SWH } & 17985.611 \mathrm{~Hz} \\ \text { FIDRES } & 0.274439 \mathrm{~Hz}\end{array}$

$\begin{array}{cc}\text { AQ } & 1.8219508 \mathrm{sec} \\ \text { AG } & 8192\end{array}$

$\begin{array}{lc}\text { JW } & 27.800 \text { usec } \\ \text { JE } & 6.00 \mathrm{usec} \\ \text { TE } & 297.6 \mathrm{~K}\end{array}$

$\begin{array}{ll}01 & 2.00000000 \mathrm{sec}\end{array}$

$\begin{array}{ll}\mathrm{d} 11 & 0.03000000 \mathrm{sec} \\ \mathrm{d12} & 0.00002000 \mathrm{sec}\end{array}$

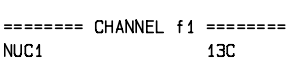

$\begin{array}{lr}P 1 & 9.40 \text { usec } \\ P L 1 & -1.00 \mathrm{~dB} \\ \text { PL1 } & 75.4752053 \mathrm{MHz}\end{array}$

$=======$ CHANNEL f2 $====== \pm$

$\begin{array}{lr}\text { CPDPRG2 waltz16 } \\ \text { VUC2 } & 1 \mathrm{H}\end{array}$

$\begin{array}{ll}\text { PCPC2 } & 1 \mathrm{H} \\ \text { PLD } & -1.00 \mathrm{usec} \\ \text { PL2 } & -1.00 \mathrm{~dB}\end{array}$

$\begin{array}{lr}\text { PL12 } & 18.00 \mathrm{~dB} \\ \text { PL13 } & 18.00 \mathrm{~dB} \\ \text { SF } & 300.1312005 \mathrm{MHZ}\end{array}$

F2 - Processing parameters
SI
SI

$\begin{array}{lc}\text { SF } & 75.4677867 \mathrm{MHz} \\ \text { WOW } & \mathrm{EM} \\ \text { SSB } & 0\end{array}$

$\begin{array}{lc}\text { SSB } & 0 \\ \angle B & 1.00 \mathrm{~Hz} \\ G B & 0\end{array}$

10 NMP p plot parameters
$22.00 \mathrm{~cm}$

$\begin{array}{lr}\mathrm{CY} & 10.00 \mathrm{~cm} \\ \mathrm{~F} & 190.000 \mathrm{pmm} \\ =1 \mathrm{P} & 14338.88 \mathrm{~Hz}\end{array}$

$\begin{array}{lr}F-1 & 14338.88 \mathrm{~Hz} \\ =2 \mathrm{P} & -5.000 \mathrm{ppm}\end{array}$

$\begin{array}{ll}\text { F2 } & -377.34 \mathrm{~Hz} \\ \text { PPMCM } & 8.86364 \mathrm{ppm} / \mathrm{cm}\end{array}$ 
2d- ${ }^{1} \mathrm{H}-\mathrm{NMR}$
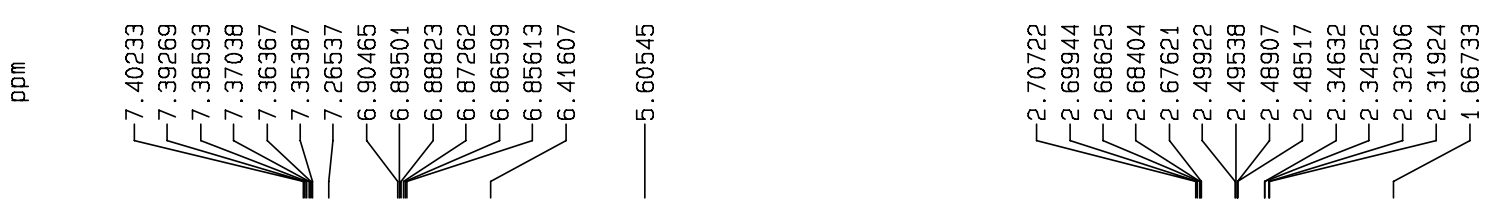

Current Data Parameters

EXPNO 20

F2 - Acquisition Parameters

Jate 20051227
16.27

INSTRUM av300

$5 \mathrm{~mm}$ DUL $13 \mathrm{C}-1$

\begin{tabular}{lr} 
PULPROG & 2930 \\
TD & 65536 \\
\hline
\end{tabular}

SOLVENT CDC13

$\begin{array}{lr}\text { NS } & 16 \\ \text { DS } & 2 \\ \text { SWH } & 6172.839 \\ \text { S }\end{array}$

$\begin{array}{ll}\text { SWH } & 6172.839 \mathrm{~Hz} \\ \text {-IDRES } & 0.094190 \mathrm{~Hz}\end{array}$

$\begin{array}{ll}\text { IDAES } & 0.094190 \mathrm{~Hz} \\ A Q & 5.3084660 \mathrm{sec}\end{array}$ $\begin{array}{lr}\text { PG } & 456.1 \\ \text { JW } & 81.000 \text { usec } \\ \text { DE } & 6.00 \text { usec }\end{array}$

$\begin{array}{cc}\text { TE } & 295.3 \mathrm{~K} \\ \text { J1 } & 2.00000000 \mathrm{sec}\end{array}$

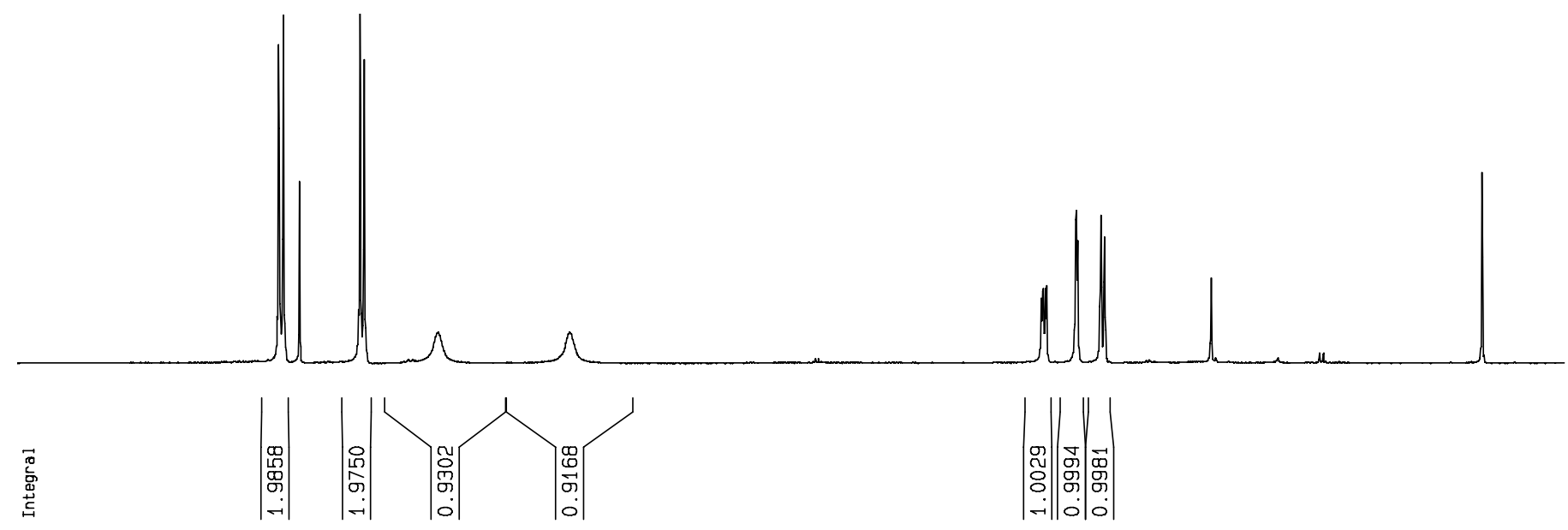

$======$ CHANNEL $f 1=======$
NUC1 $1 \mathrm{H}$

$1 \quad-9.30$ us

$\begin{array}{lr}-1.00 \mathrm{~dB} \\ \text { SF01 } & 300.1318534 \mathrm{MHz}\end{array}$

=2 - Processing parameters

SI 32768

$\begin{array}{ll}\text { SF } & 300.1300047 \mathrm{MHz} \\ \text { WOW } & \end{array}$

$\begin{array}{lc}\text { WDW } & E M \\ \text { SSB } & 0 \\ \text { LB } & 0.30 \mathrm{~Hz}\end{array}$

$\begin{array}{lr}\mathrm{GB} & 0 \\ \mathrm{PC} & 1.00\end{array}$

10 NMF plot parameters

CX $\quad 22.00 \mathrm{~cm}$

$\begin{array}{lr}C Y & 5.00 \mathrm{~cm} \\ =1 \mathrm{P} & 9.000 \mathrm{ppm}\end{array}$

$\begin{array}{lr}=1 \mathrm{P} & 9.000 \mathrm{ppm} \\ =1 & 2701.17 \mathrm{~Hz}\end{array}$

$\begin{array}{ll}-1 & -0.500 \mathrm{ppm} \\ -2 \mathrm{P} & -150.07 \mathrm{~Hz} \\ =2 & 0.45182 \mathrm{pm}\end{array}$

PPMCM $\quad 0.43182 \mathrm{Dpm} / \mathrm{cm}$

HZCM $\quad 129.60159 \mathrm{~Hz} / \mathrm{cm}$

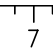

6

5

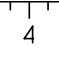

2

1 
2d- ${ }^{13} \mathrm{C}-\mathrm{NMR}$

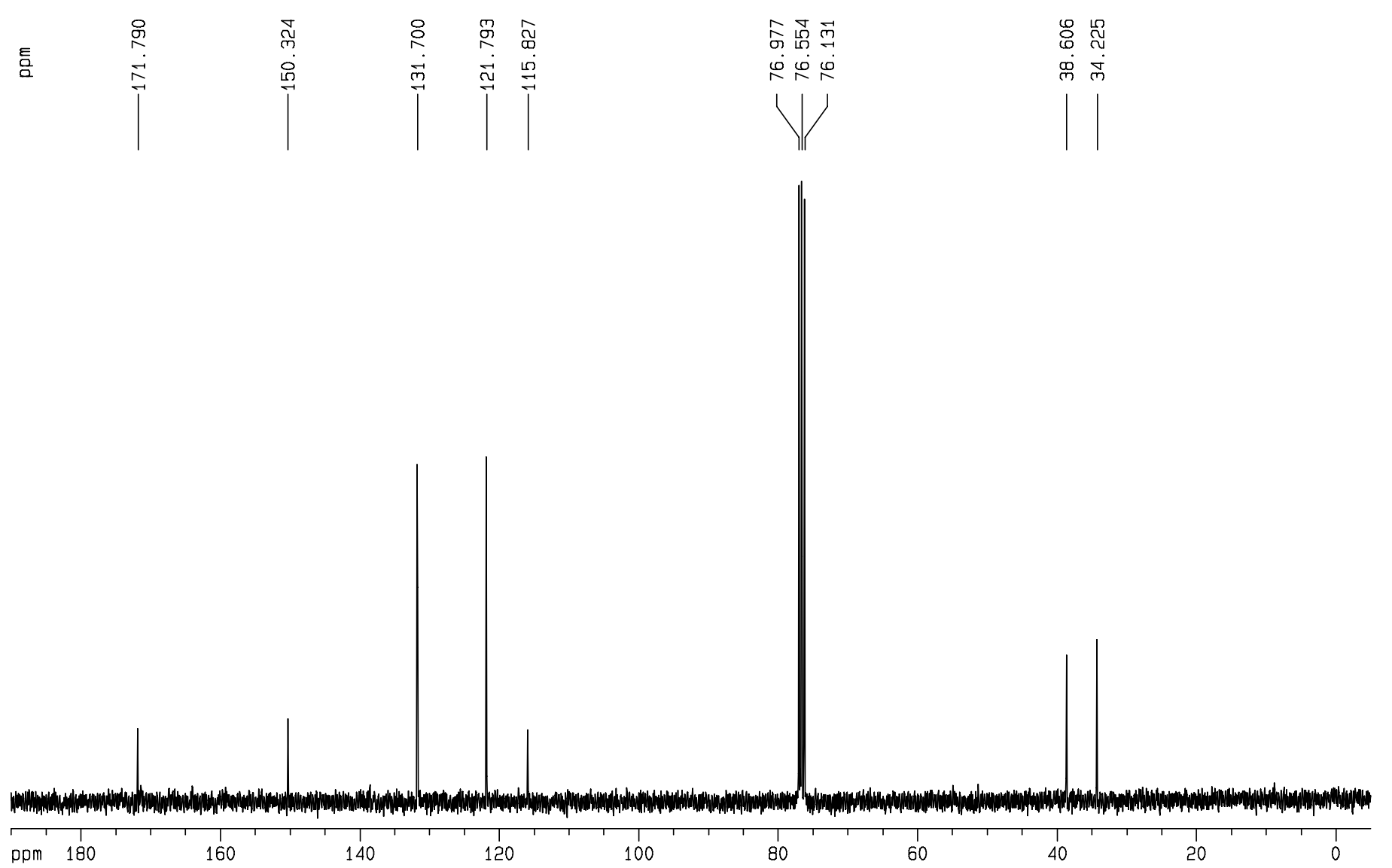

Current Data Parameters

AME wiy05-1227a

PROCNO

F2 - Acquisition Parameters

Jime

$5 \mathrm{~mm}$ OUL $13 \mathrm{c} 30-1$

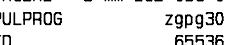

$\begin{array}{lr}\text { TOLVENT } & \text { COC13 } \\ \text { SS } & 140 \\ \text { NS } & 4950\end{array}$

$\begin{array}{lr}\text { SWH } & 4 \\ \text { SW } & 17985.611 \mathrm{~Hz}\end{array}$

AQ $\quad 1.8219508 \mathrm{sec}$

$\begin{array}{ll}286 & 2896.3 \\ \text { OW } & 27.800 \text { use }\end{array}$

$\begin{array}{lr}5.00 \mathrm{usec} \\ \mathrm{TE} & 294.7 \mathrm{~K} \\ \mathrm{TE} & 2.000000 \mathrm{sec}\end{array}$

$\begin{array}{ll}\mathrm{J} 1 & 2.00000000 \mathrm{sec} \\ \mathrm{d11} & 0.03000000 \mathrm{sec}\end{array}$

$==3=z=$ CHANNEL $f 1$ =z=z=z=

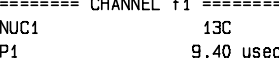

$\begin{array}{lr} & 9.40 \text { usec } \\ \text { PL1 } & -1.00 \mathrm{~dB} \\ \text { SF } & 75.4752953 \mathrm{MHZ}\end{array}$

$======$ CHANNEL $f 2=== \pm===$

$\begin{array}{lr}\text { CPDPRG2 } & \text { waltz16 } \\ \text { NUC2 } & 1 \mathrm{H}\end{array}$

$\begin{array}{lr}\text { NUC2 } & 1 \mathrm{H} \\ \text { PCPD2 } & 80.00 \mathrm{usec} \\ \text { PL2 } & -1.00 \mathrm{~dB}\end{array}$

$\begin{array}{ll}18.00 \mathrm{~dB} \\ \mathrm{R} 12 & 18.00 \mathrm{~dB}\end{array}$

$\begin{array}{ll}18.00 \mathrm{~dB} \\ \mathrm{SF} 02 & 300.1312005 \mathrm{MHZ}\end{array}$

$=2$ - Processing parameters

SI Processing parameters

$\begin{array}{lc}\text { SF } & 75.4677867 \mathrm{MHz} \\ \text { WOW } & \mathrm{EM}\end{array}$

$\begin{array}{lc}\mathrm{SSB} & 0 \\ \mathrm{LB} & 1.00 \mathrm{~Hz} \\ \mathrm{~GB} & 0\end{array}$

10 NMP plot parameters
22.00

$\begin{array}{lr}\mathrm{CY} & 10.00 \mathrm{~cm} \\ \mathrm{~F} & 190.000 \mathrm{pmm} \\ =1 \mathrm{P} & 1438.08 \mathrm{~Hz}\end{array}$

$14338.88 \mathrm{~Hz}$
$-5.000 \mathrm{ppm}$

$-377.34 \mathrm{~Hz}$

$8.86364 \mathrm{ppm} / \mathrm{cm}$
$668.91901 \mathrm{~Hz} / \mathrm{cm}$ 


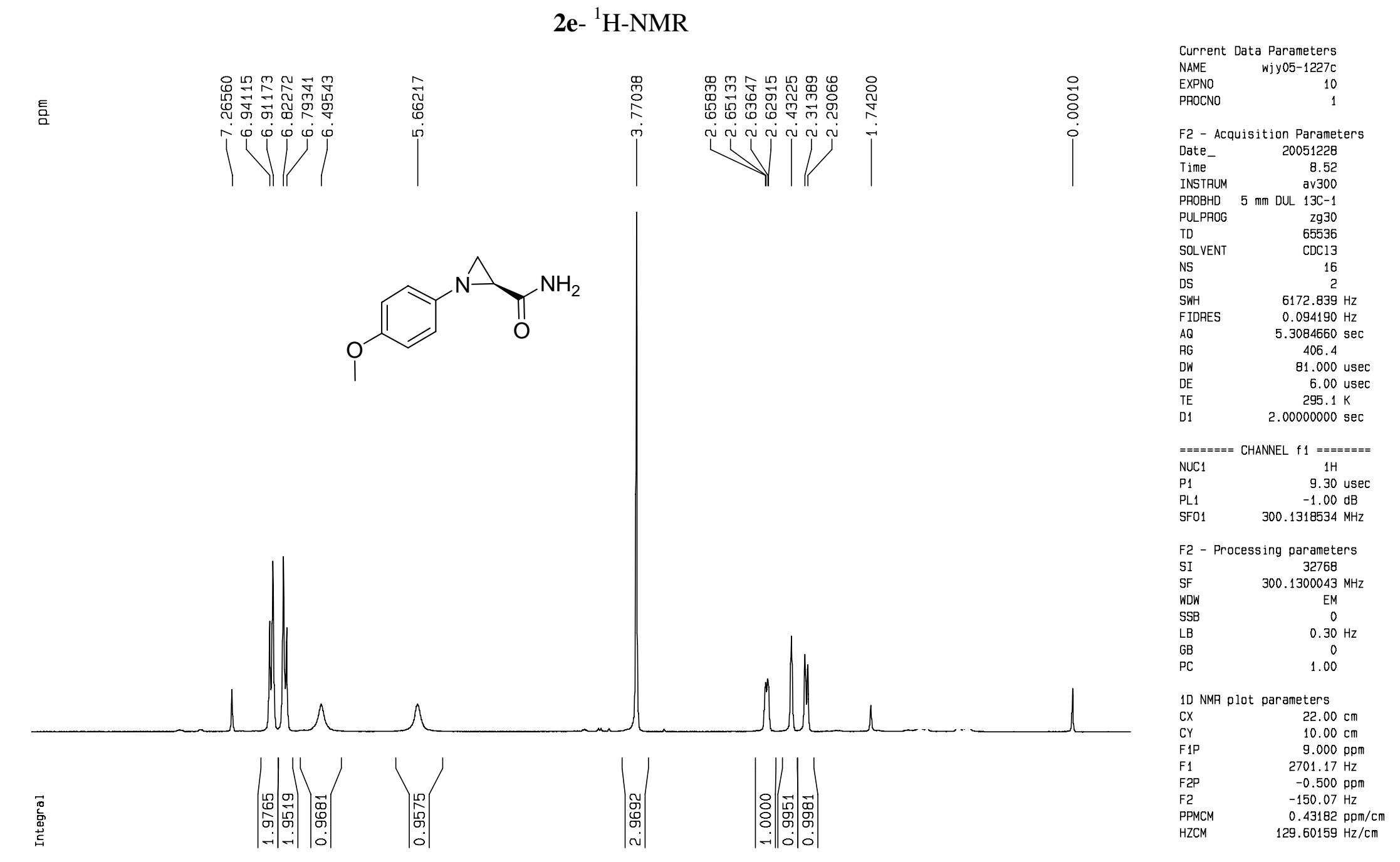


2e- ${ }^{13} \mathrm{C}-\mathrm{NMR}$

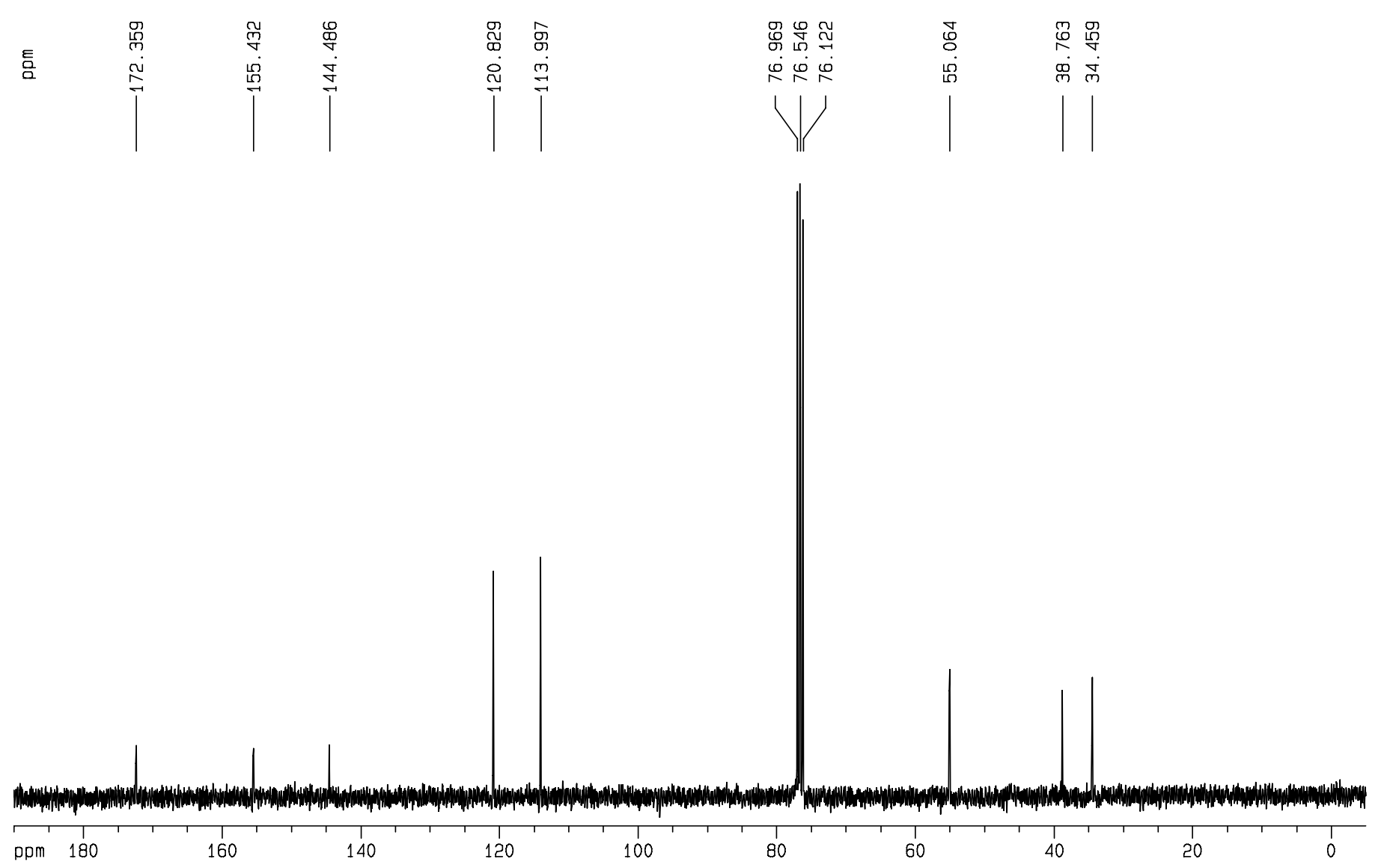

Current Data Parameters

EXPNO WJy05-1227c

PROCNO

F2 - Acquisition Parameters

Date
Time

गRОВНD $5 \mathrm{~mm}$ DUL $\begin{aligned} & \text { av300 } \\ & 13 \mathrm{C}-1\end{aligned}$

PULPROG 29p930

$\begin{array}{lr}\text { TS } & 65536 \\ \text { SOLVENT } & \text { COCI3 } \\ \text { NS } & 137\end{array}$

SWH $\quad 17985.611 \mathrm{~Hz}$

$\begin{array}{ll} & \\ \text { FIDRES } & 0.274439 \mathrm{~Hz}\end{array}$

$\begin{array}{cc}\text { AQ } & 1.8219508 \mathrm{sec} \\ \text { AG } & 4096\end{array}$

$\begin{array}{lc}\mathrm{JW} & 27.800 \mathrm{usec} \\ \mathrm{OW} & 6.00 \mathrm{usec} \\ \mathrm{DE} & 295.1 \mathrm{~K}\end{array}$

$\begin{array}{ll}01 & 2.00000000 \mathrm{sec}\end{array}$

$\begin{array}{ll}\mathrm{d} 11 & 0.03000000 \mathrm{sec} \\ \mathrm{d} 12 & 0.00002000 \mathrm{sec}\end{array}$

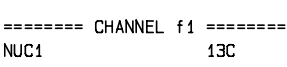

$\begin{array}{lr}P 1 & 9.40 \text { usec } \\ \text { PL1 } & -1.00 \mathrm{~dB} \\ \text { PL } & 75.4752053 \mathrm{MHz}\end{array}$

$=======$ CHANNEL f2 $====== \pm$

$\begin{array}{lr}\text { CPDPRG2 } & \text { waltz16 } \\ \text { VUC2 } & 1 \mathrm{H}\end{array}$

$\begin{array}{ll}\text { PCPD2 } & 80.00 \mathrm{usec} \\ \text { PL2 } & -1.00 \mathrm{~dB}\end{array}$

$\begin{array}{lr}\text { PL12 } & 18.00 \mathrm{~dB} \\ \text { PL13 } & 18.00 \mathrm{~dB} \\ \text { SF02 } & 300.1312005 \mathrm{MHZ}\end{array}$

2 - Processing parameters

F2 - Processing parameters
SI 32768

$\begin{array}{lc}\text { SF } & 75.4677867 \mathrm{MHz} \\ \text { WOW } & \mathrm{EM}\end{array}$

$\begin{array}{lc}\text { SSB } & 0 \\ L B & 1.00 \mathrm{~Hz} \\ G B & 0\end{array}$

10 NMP p lot parameters

$\begin{array}{ll}\text { CX } & 22.00 \mathrm{~cm} \\ \mathrm{CY} & 10.00 \mathrm{~cm}\end{array}$

$\begin{array}{lr}C Y & 10.00 \mathrm{~cm} \\ F 1 \mathrm{P} & 190.000 \mathrm{ppm} \\ F & 14338.80 \mathrm{~Hz}\end{array}$

$1430.00 \mathrm{pz}$
$-5.000 \mathrm{ppm}$
$-377.34 \mathrm{~Hz}$

$\begin{array}{ll}=2 & -377.34 \mathrm{~Hz} \\ \text { PPMCM } & 8.66364 \mathrm{ppm} / \mathrm{cm}\end{array}$ 


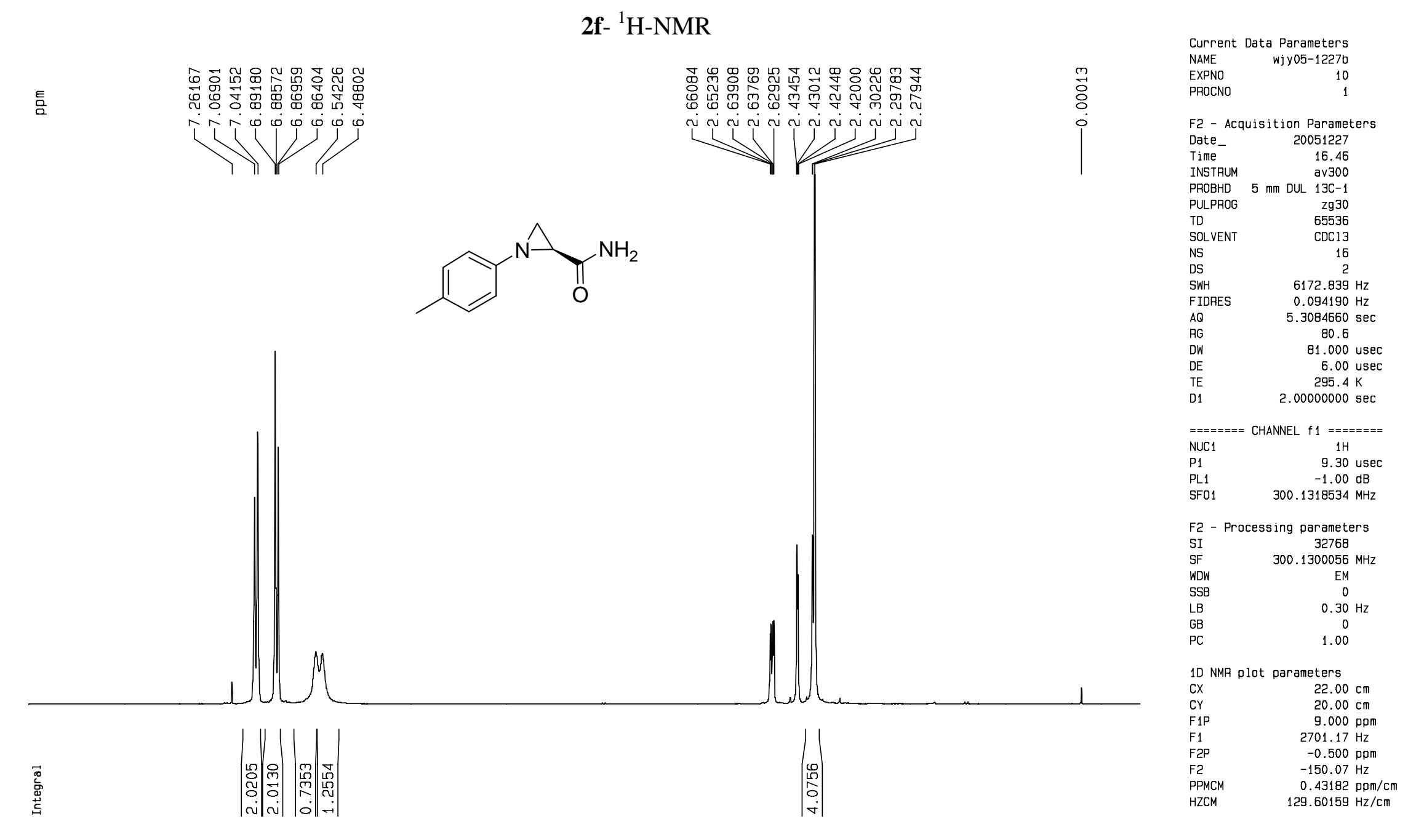


2f- ${ }^{13} \mathrm{C}-\mathrm{NMR}$

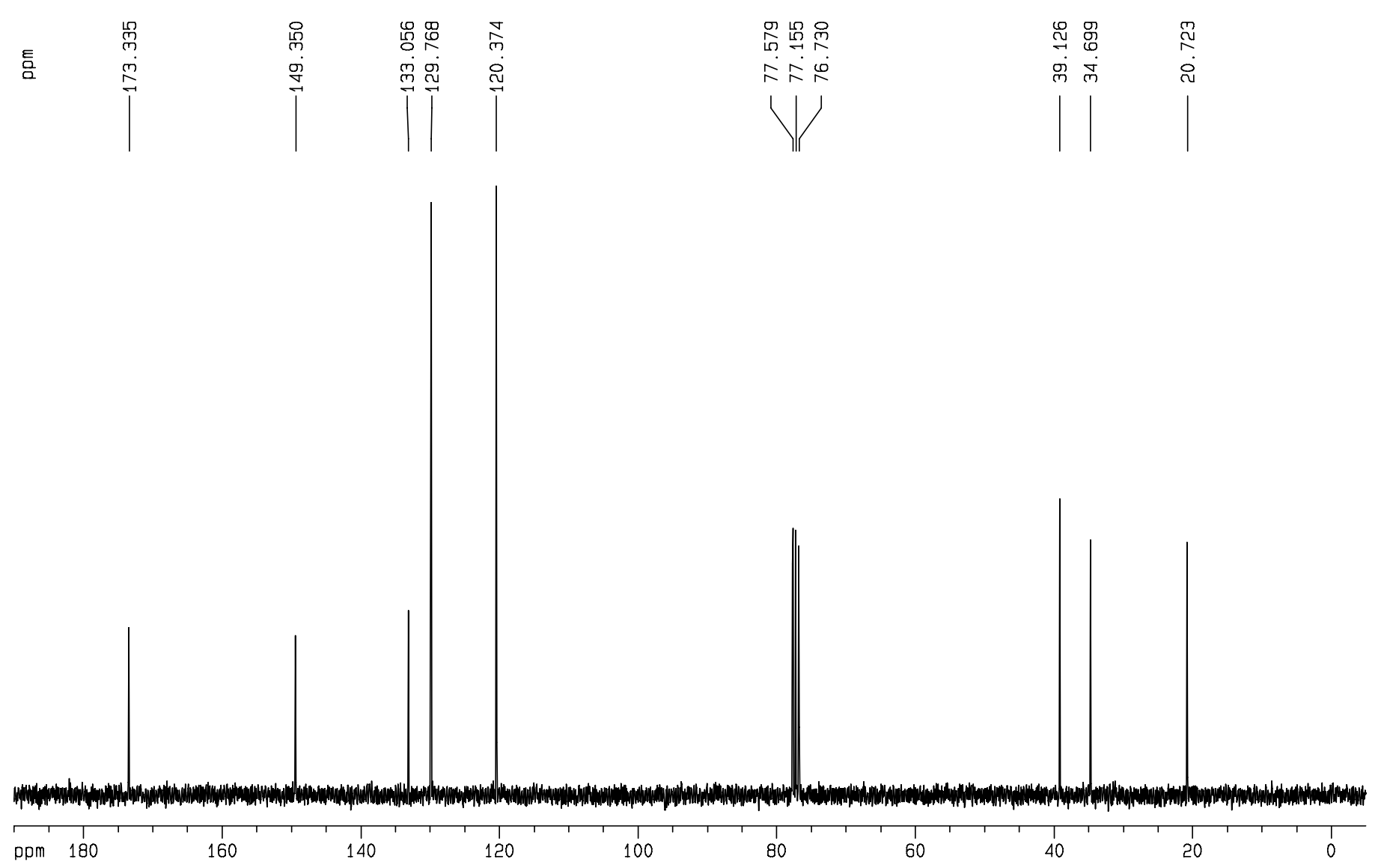

Current Data Parameters
NAME Wjy05-1227b

EXPNO

F2 - Acquisit Parameter

${ }_{\text {Tate }}$ Time

INSTRUM av300

$\begin{array}{lr}\text { TDP } & \text { 2950 } \\ \text { TD } & 65536 \\ \text { SOLVENT } & \text { CDC13 }\end{array}$

$\begin{array}{lr}\text { JS } & 4 \\ \text { SWH } & 17985.611 \mathrm{~Hz}\end{array}$

$\begin{array}{ll}\text { AQ } & 0.274439 \mathrm{~Hz} \\ \mathrm{AQ} & 1.8219508 \mathrm{sec}\end{array}$

$\begin{array}{lr}\text { RG } & 8192 \\ \text { DW } & 27.800 \text { usec }\end{array}$

$\begin{array}{lr}\text { JE } & 6.00 \text { usec } \\ \text { TE } & 295.4 \mathrm{k} \\ \text { J1 } & 20000000 \mathrm{sec}\end{array}$

$\begin{array}{ll}\mathrm{J} & 2.00000000 \mathrm{sec} \\ \mathrm{d} 11 & 0.030000000 \mathrm{sec}\end{array}$

$== \pm= \pm==$ CHANNEL $f 1$ = $= \pm= \pm= \pm$

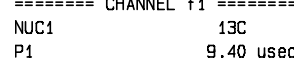

$\begin{array}{lr}\text { P1 } & 9.40 \mathrm{usec} \\ \text { PL1 } & -1.00 \mathrm{~dB} \\ \text { SF01 } & 75.4752953 \mathrm{MHZ}\end{array}$

$======$ CHANNEL $f 2=======$

POPAG2 waltz16

$\begin{array}{ll}\text { NUC2 } & 1 \mathrm{H} \\ \text { PCD2 } & 80.00 \mathrm{usec} \\ \text { PL2 } & -1.00 \mathrm{~dB}\end{array}$

$\begin{array}{ll}\text { PL12 } & 18.00 \mathrm{~dB} \\ \text { PL13 } & 18.00 \mathrm{~dB}\end{array}$

$\begin{array}{lr}\text { PL13 } & 18.00 \mathrm{~dB} \\ \text { SF } 02 & 300.1312005 \mathrm{MHZ}\end{array}$

$\begin{array}{lr}\text { F2 - Processing parameters } \\ \text { SI } & 32768 \\ \text { SF } & 75.4677490 \\ \text { MHZ }\end{array}$

$\begin{array}{lr}\text { SF } & 75.4677490 \\ \text { WOW } & \text { EM } \\ \text { SSB } & 0\end{array}$

$\begin{array}{ll}\angle B & 0 \\ G B & 1.00 \mathrm{~Hz}\end{array}$

10 NMP p lot parameters
$22.00 \mathrm{~cm}$

$\begin{array}{lr}C Y & 10.00 \mathrm{~cm} \\ F 1 \mathrm{P} & 190.000 \mathrm{ppm} \\ =1 & 14338.87 \mathrm{~Hz}\end{array}$

$-5.000 \mathrm{ppm}$

$-377.34 \mathrm{~Hz}$

$8.86364 \mathrm{ppm} / \mathrm{cm}$
$668.91870 \mathrm{~Hz} / \mathrm{cm}$ 
2g- ${ }^{1} \mathrm{H}-\mathrm{NMR}$

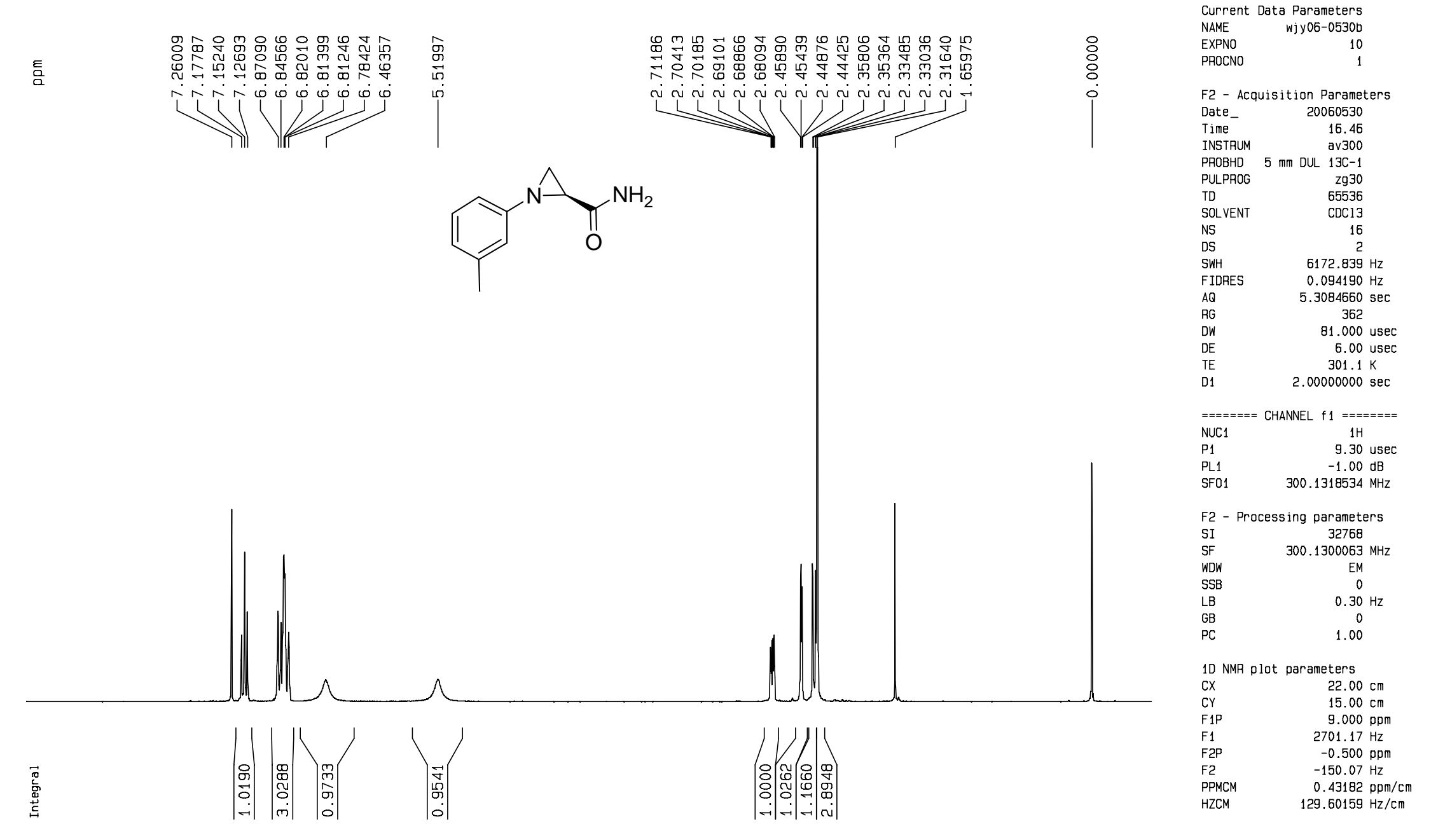


2g- ${ }^{13} \mathrm{C}-\mathrm{NMR}$
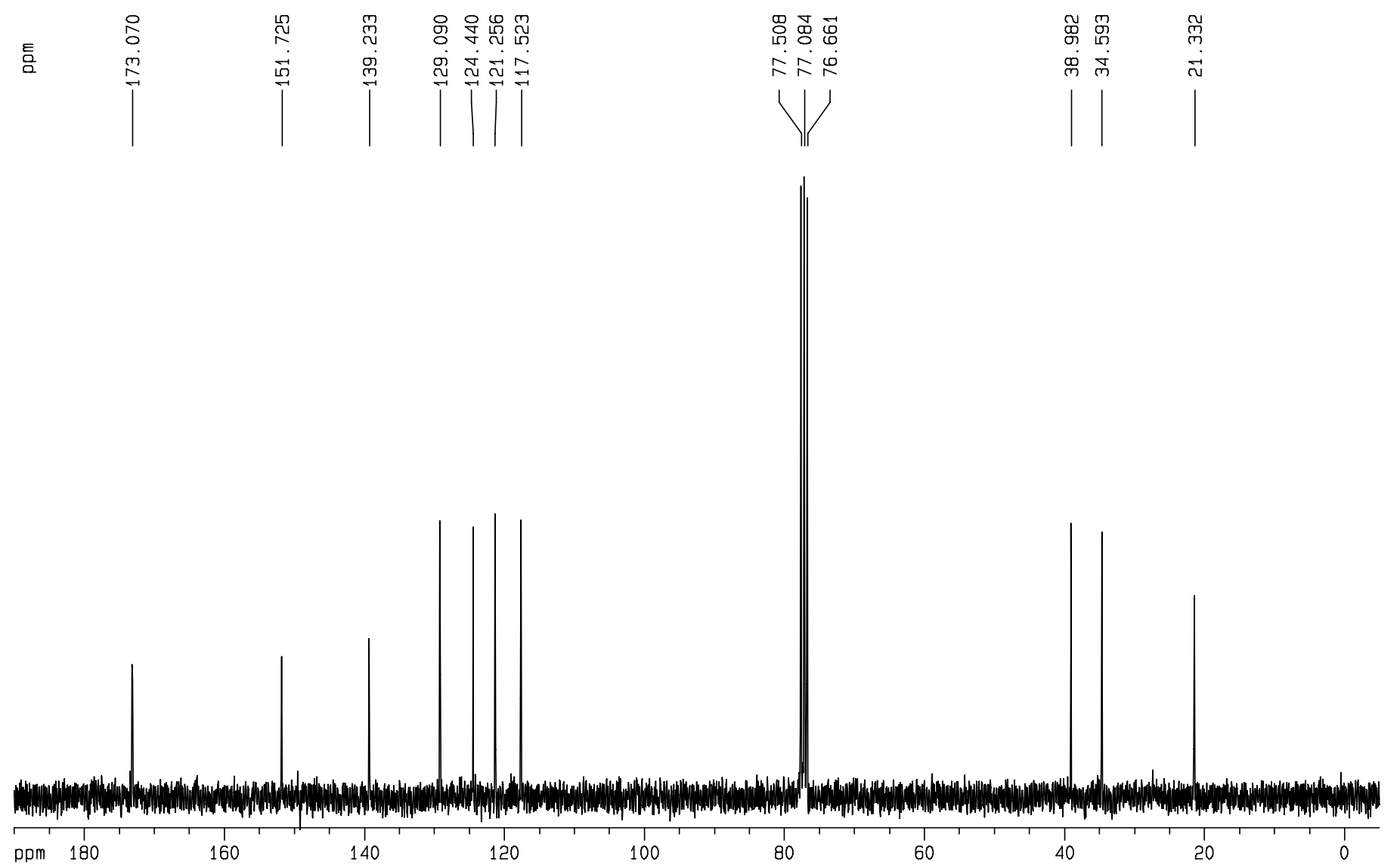

Current Data Parameters

EXPNO

PROCNO

F2 - Acquisition Parameters

Jate

Jate
Time
INSTRUM

5 mm DUL $13 C^{-1}$

PULPROG 29P950

$\begin{array}{lrl}\text { SOLVENT } & \text { COCI3 } \\ \text { NS } & 69\end{array}$

$\begin{array}{ll}\text { SWH } & 4 \\ \text { SWH } & 17985.611 \mathrm{~Hz}\end{array}$

AQ $\quad 1.8219508 \mathrm{sec}$

$\begin{array}{ll}\mathrm{AG} & 5792.6 \\ \mathrm{DW} & 27.800 \text { usec }\end{array}$

$\begin{array}{cc}\text { JE } & 6.00 \text { usec } \\ \text { TE } & 297.3 \mathrm{k} \\ \mathrm{T} & 2.00000000 \mathrm{sec}\end{array}$

$\begin{array}{ll}01 & 2.00000000 \mathrm{sec} \\ \mathrm{d} 11 & 0.03000000 \mathrm{sec} \\ \mathrm{d12} & 0.00002000\end{array}$

$== \pm= \pm==$ CHANNEL $f 1$ = $= \pm= \pm= \pm$

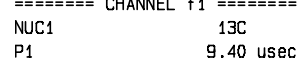

$\begin{array}{lr}\text { P1 } & 9.40 \text { usec } \\ \text { PL1 } & -1.00 \mathrm{~dB} \\ \text { SF01 } & 75.4752953 \mathrm{MHZ}\end{array}$

$======$ CHANNEL $f 2=======$

$\begin{array}{lr}\text { CPOPAG2 } & \text { waltz16 } \\ \text { VUC2 } & 1 \mathrm{H}\end{array}$

$\begin{array}{ll}\text { PCPD2 } & 80.00 \text { usec } \\ \text { PL2 } & -1.00 \mathrm{~dB}\end{array}$

$\begin{array}{lr}\text { PL12 } & 18.00 \mathrm{~dB} \\ \text { PL13 } & 18.00 \mathrm{~dB} \\ \text { SF02 } & 300.1312005 \mathrm{MHZ}\end{array}$

F2 - Processing parameters
SI

$\begin{array}{lr}\text { SI } & 32768 \\ \text { SF } & 75.4677490 \\ \mathrm{MHZ}\end{array}$

$\begin{array}{lr}\text { WF } & 75.4677490 \\ \text { WOW } & \text { EM } \\ \text { SSB } & 0 \\ \text { B } & 1.00\end{array}$

$\begin{array}{ll}\mathrm{SB} & 1.00 \\ \mathrm{IB} & 0 \\ \mathrm{PC} & 1.40\end{array}$

10 NMR p plot parameters
CX $22.00 \mathrm{~cm}$

$\begin{array}{lr}\mathrm{CY} & 10.00 \mathrm{~cm} \\ F 1 \mathrm{P} & 190.000 \mathrm{pmm} \\ =1 & 14338.87 \mathrm{~Hz}\end{array}$

$\begin{array}{ll}-2 \mathrm{P} & 14338.87 \mathrm{~Hz} \\ =25 & -5.000 \mathrm{ppm}\end{array}$

$\begin{array}{ll}\text { F2 } & -377.34 \mathrm{~Hz} \\ \text { PPMCM } & 8.86364 \mathrm{ppm} / \mathrm{cm}\end{array}$ 


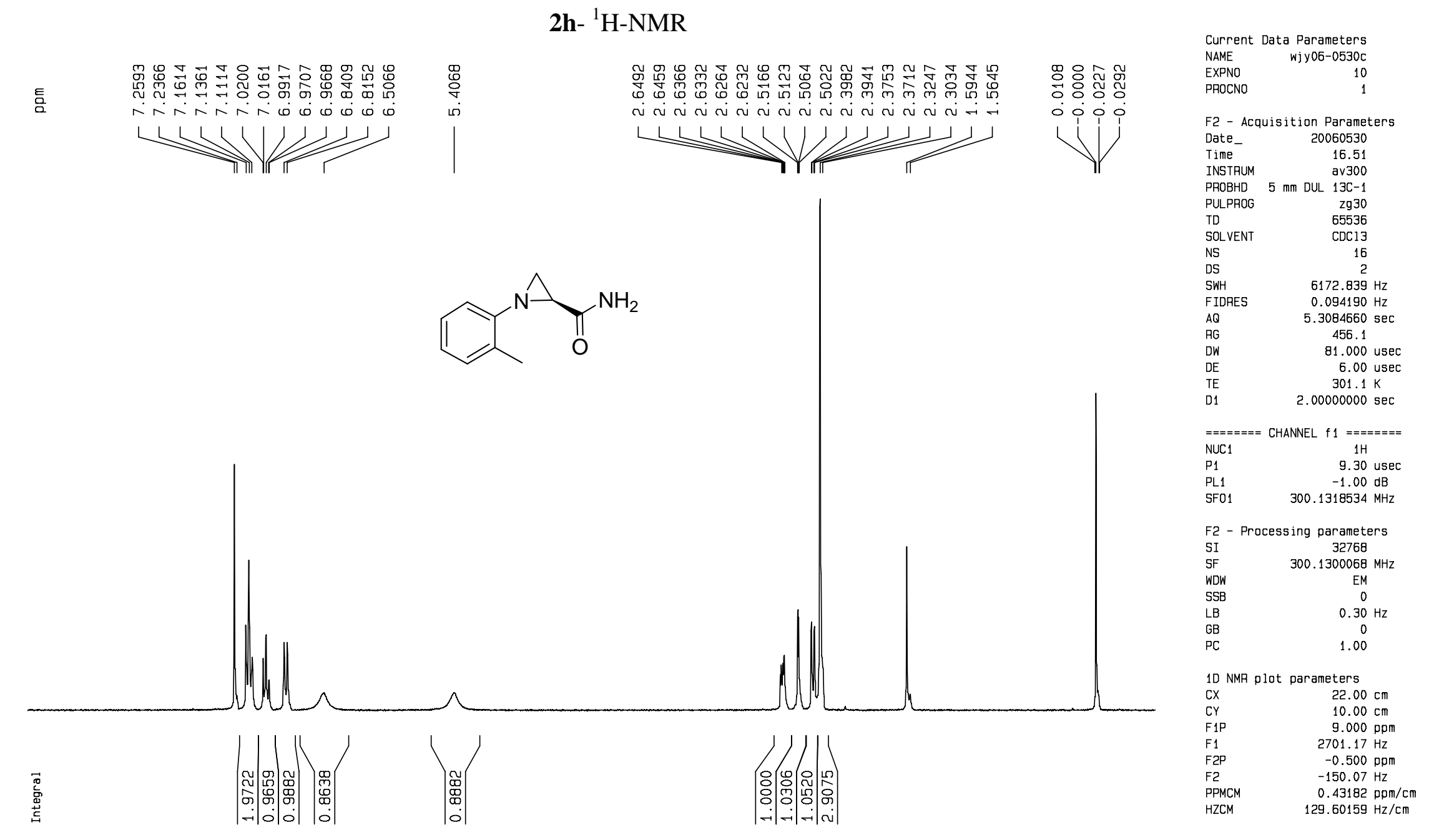


2h- ${ }^{13} \mathrm{C}-\mathrm{NMR}$

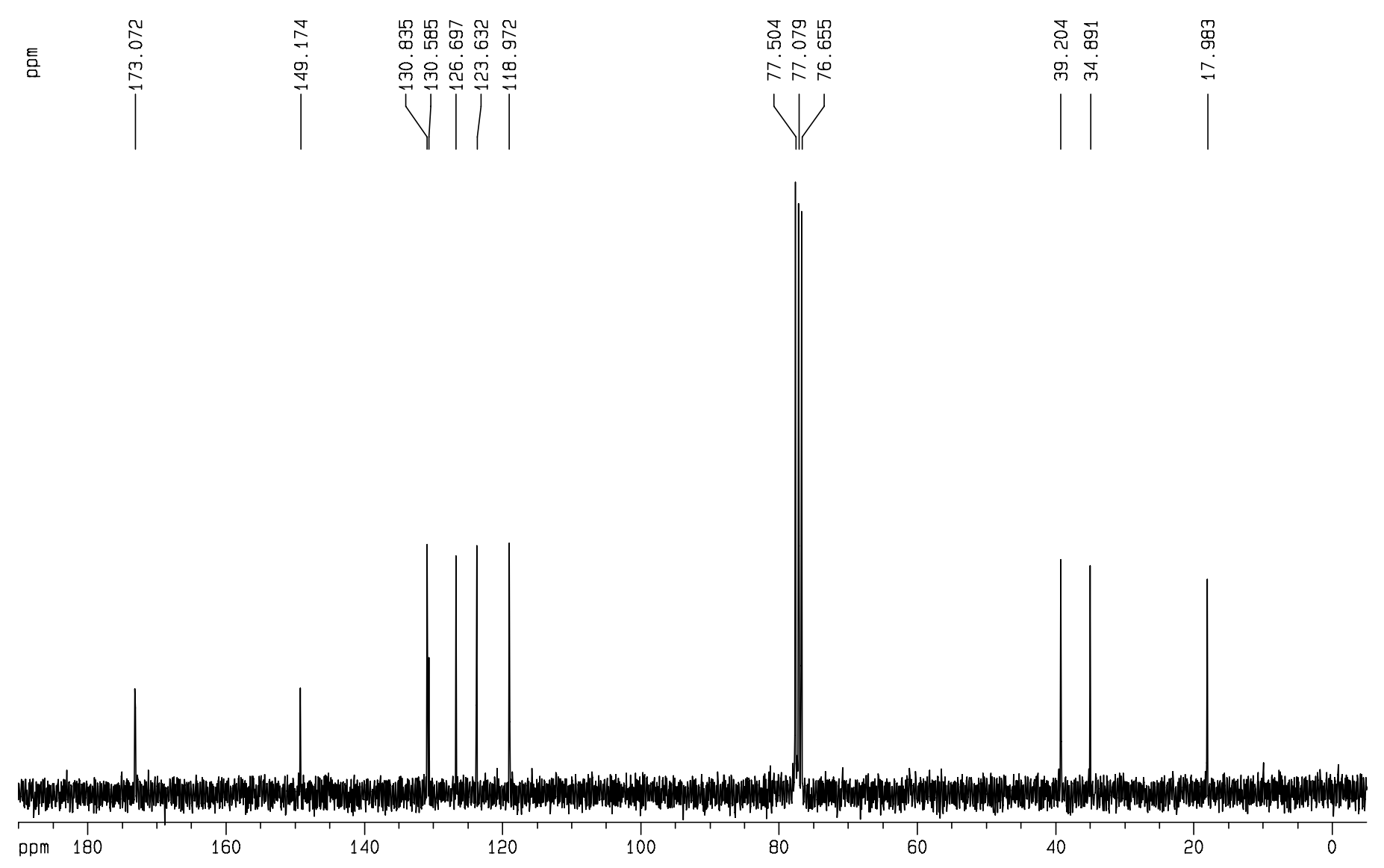

Current Data Parameters
NAME Wjy06-0112b

EXPNO WJYO6-01120

PROCNO

F2 - Acquisition Parameters

Date
Time

PROBHD $5 \mathrm{~mm}$ DUL $13 \mathrm{CV}-1$

PULPROG $\quad$ 29P930

$\begin{array}{lr}\text { SOLVENT } & \text { COC13 } \\ \text { NS } & 67\end{array}$

$\begin{array}{lr}\text { SW } & 4 \\ \text { SWH } & 17985.611 \mathrm{~Hz}\end{array}$

FIDRES $\quad 0.274439 \mathrm{~Hz}$
AQ

$\begin{array}{ll}\text { AQ } & 1.8219508 \mathrm{sec} \\ \mathrm{AG} & 5792.6\end{array}$

$\begin{array}{lc}\text { JW } & 27.800 \text { usec } \\ \text { DE } & 6.00 \mathrm{usec} \\ \mathrm{TE} & 297.2 \mathrm{~K}\end{array}$

$\begin{array}{ll}\text { J1 } & 2.00000000 \mathrm{sec} \\ \mathrm{d} 11 & 0.03000000 \mathrm{sec}\end{array}$

$\begin{array}{ll}\begin{array}{l}\mathrm{d} 11 \\ \mathrm{~d}\end{array} & 0.03000000 \mathrm{sec} \\ \mathrm{d12} & 0.00002000 \mathrm{sec}\end{array}$

$=======$ CHANNEL
NUC1 1
NuC==z=="
$13 \mathrm{C}$

$\begin{array}{lr}\text { P1 } & 9.40 \text { usec } \\ \text { PL1 } & -1.00 \mathrm{~dB} \\ \text { PF } & 75.4752953 \mathrm{MHz}\end{array}$

$=======$ CHANNEL f2 $=======$

$\begin{array}{lc}\text { CPDPRG2 } & \text { Waltz16 } \\ \text { NUC2 } & 1 \mathrm{H} \\ \text { PCPD2 } & 80.00 \mathrm{usec} \\ \text { PL2 } & -1.00 \mathrm{~dB} \\ \text { PL12 } & 18.00 \mathrm{~dB} \\ \text { PL13 } & 18.00 \mathrm{~dB} \\ \text { SF02 } & 300.1312005 \mathrm{MHZ}\end{array}$

F2 - Processing parameters
SI
32768

$\begin{array}{ll}\text { SI } & 32768 \\ \text { SF } & 75.4677490 \mathrm{MHz}\end{array}$

$\begin{array}{lr}\text { SF } & 75.4677490 \\ \text { WOW } & \text { EM } \\ \text { SSB } & 0 \\ \text { LB } & 1.00\end{array}$

$\begin{array}{ll} & 1.00 \\ \mathrm{SB} & 0 \\ \mathrm{PC} & 1.40\end{array}$

$10 \mathrm{NMF}$ plot parameters
$\mathrm{CX}$
$22.00 \mathrm{~cm}$

$\begin{array}{lr}\mathrm{CY} & 10.00 \mathrm{~cm} \\ \mathrm{~F} 1 \mathrm{P} & 190.000 \mathrm{pmm} \\ \mathrm{F} & 14338.87 \mathrm{~Hz}\end{array}$

$14338.87 \mathrm{~Hz}$
$-2 \mathrm{P}$
$-5.000 \mathrm{ppm}$

$\begin{array}{ll}\text { F2 } & -377.34 \mathrm{~Hz} \\ \text { PPMCM } & 8.86364 \mathrm{ppm} / \mathrm{cm}\end{array}$ 
4a- ${ }^{1} \mathrm{H}-\mathrm{NMR}$
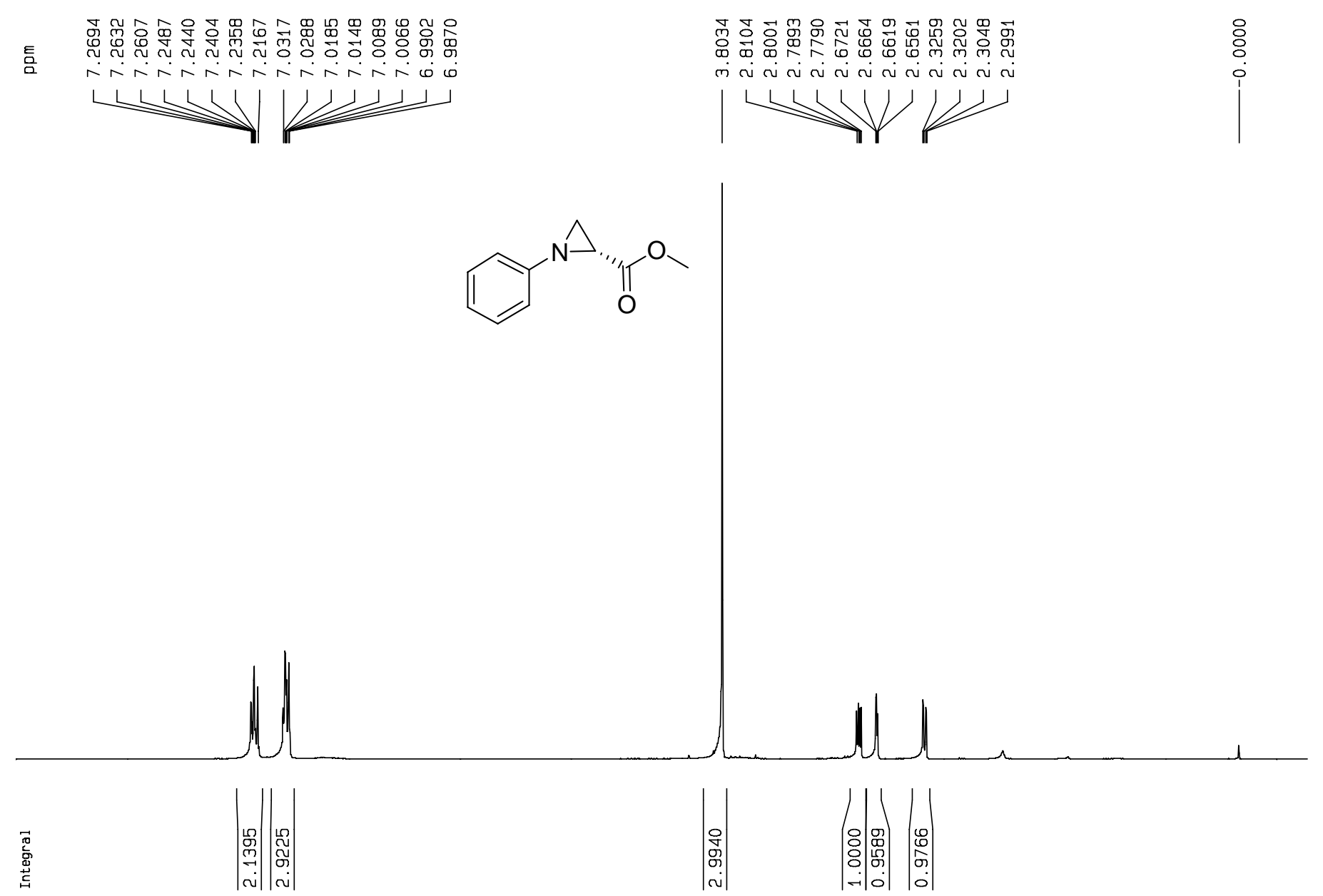

Current Data Parameters NAME wjy05-0922a EXPNO
PROCNO

F2 - Acquisition Parameter

Date_ 20050923

Time

21.54
av300

PULPROG 5 IIII DUL 13C-1

TD $\quad 65536$

$\begin{array}{lr}\text { SOLVENT } & \text { CDC13 } \\ \text { DS } & 16\end{array}$

$\begin{array}{lr}\text { DS } & 2 \\ \text { SWH } & 6172.839 \mathrm{~Hz}\end{array}$

IDRES $\quad 0.094190 \mathrm{~Hz}$

$\quad 5.3084660 \mathrm{sec}$

$\begin{array}{lr}\text { RG } & 128 \\ \text { DW } & 81.000 \text { usec } \\ \text { DE } & 6.00 \text { usec }\end{array}$

$299.9 \mathrm{~K}$

D1 $2.00000000 \mathrm{sec}$

$=======$ CHANNEL $f 1=======$
NUC1

$\begin{array}{ll}\text { NUC1 } & 1 \mathrm{H} \\ \mathrm{P} 1 & 9.30 \mathrm{usec}\end{array}$

$\begin{array}{lr}\text { PL1 } & -1.00 \mathrm{~dB} \\ \text { SF01 } & 300.1318534 \mathrm{MHz}\end{array}$

F2 - Processing parameters

SI Processing parameters

$\begin{array}{lc}\text { SF } & 300.1300060 \mathrm{MHZ} \\ \text { WOW } & \mathrm{EM}\end{array}$

$\begin{array}{lc}\text { SSB } & 0 \\ \text { LB } & 0.30 \mathrm{~Hz} \\ G B & 0\end{array}$

$\begin{array}{lr}G B & 0 \\ P C & 1.00\end{array}$

10 NMP plot parameters

$\begin{array}{ll}\text { CX } & 22.00 \mathrm{~cm} \\ \mathrm{CY} & \end{array}$

$\begin{array}{ll}\text { FIP } & 9.000 \mathrm{~cm} \\ \text { FI } & 9.017 \mathrm{~mm}\end{array}$

$\begin{array}{ll}F 1 & 2701.17 \mathrm{~Hz} \\ \text { F2P } & -0.500 \mathrm{PPR}\end{array}$

F2 $\quad-0.500 \mathrm{ppm}$

$\begin{array}{lr}\text { PPMCM } & 0.43182 \mathrm{ppm} / \mathrm{cm} \\ \text { HZCM } & 129.60159 \mathrm{~Hz} / \mathrm{cm}\end{array}$ 
4b- ${ }^{1} \mathrm{H}-\mathrm{NMR}$

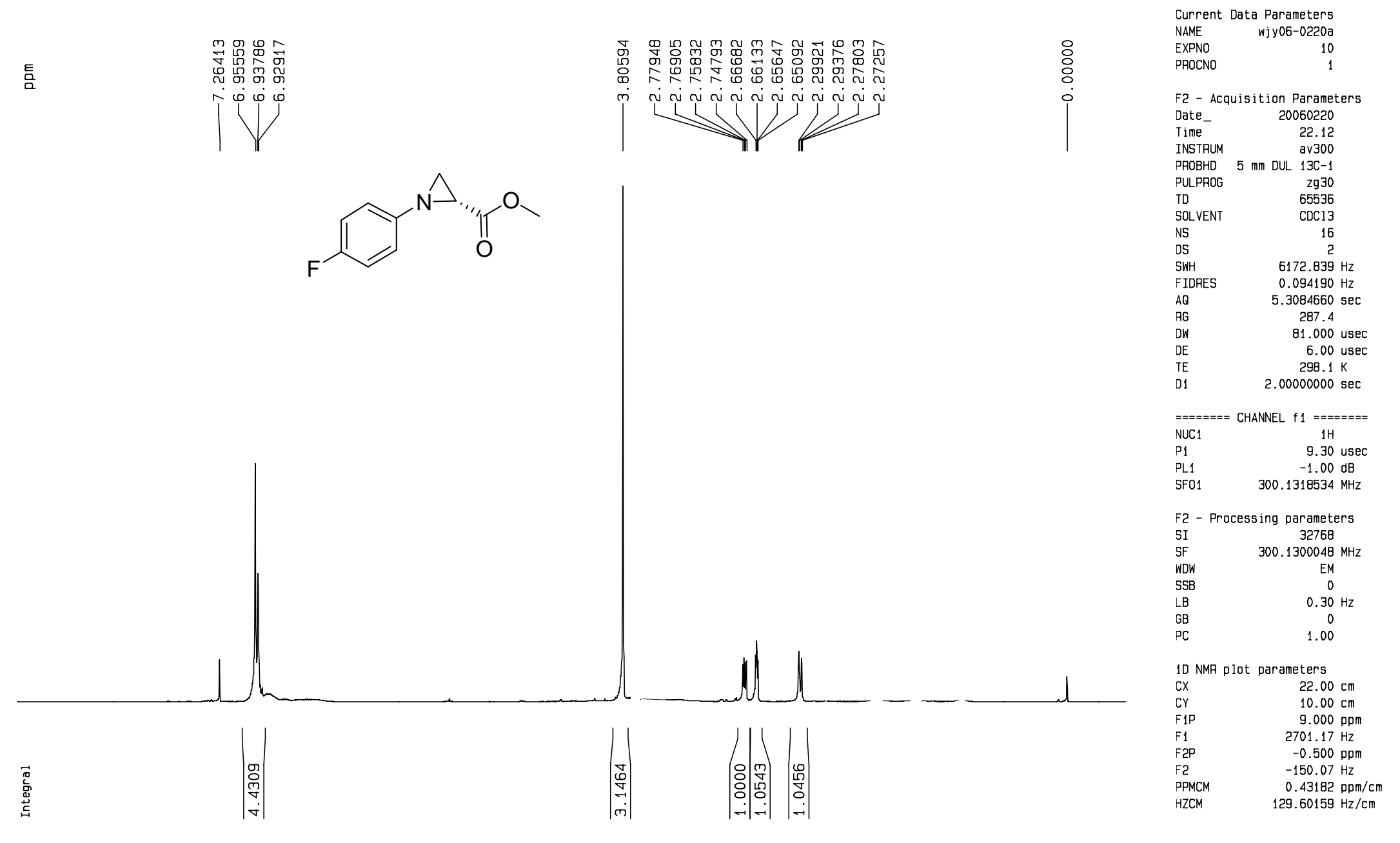


4c- ${ }^{1} \mathrm{H}-\mathrm{NMR}$

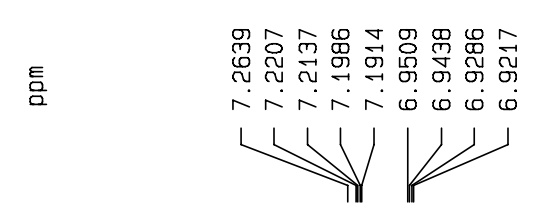

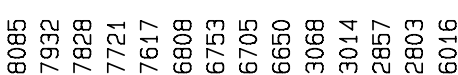

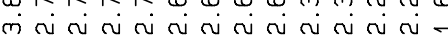

14

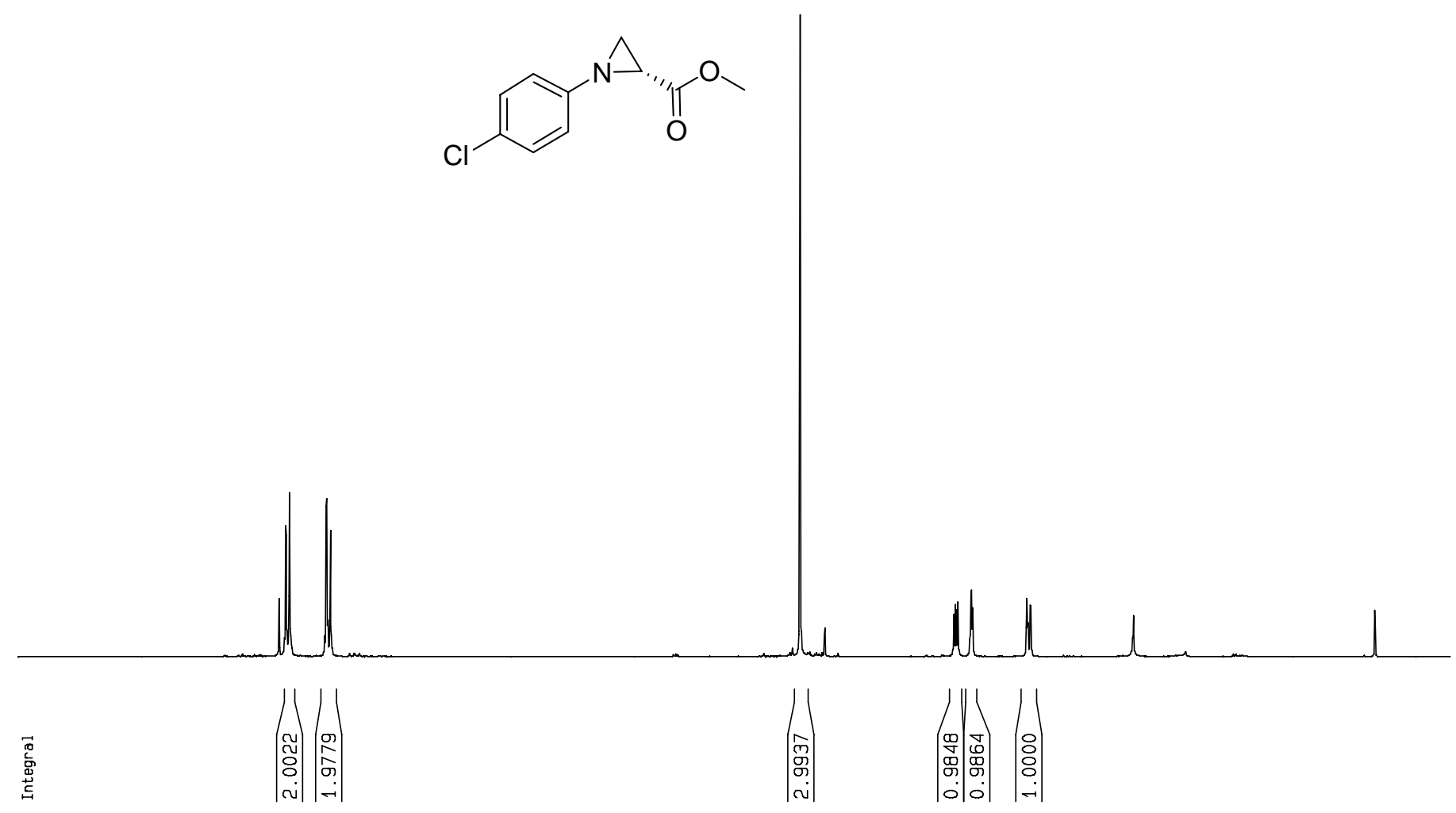

Current Data Parameters

NAME wjy 05-1102a

PROCNO

F2 - Acquisition Parameters

Tate 20051102
16.01

INSTRUM av300 $5 \mathrm{~mm}$ DUL $13 \mathrm{C}-1$ \begin{tabular}{lr} 
PULPROG & 2930 \\
TD & 65536 \\
\hline 50 & CDC1
\end{tabular}

$\begin{array}{lr}\text { SOLVENT } & \text { CDC13 } \\ \text { NS } & 16\end{array}$

$\begin{array}{lr}\text { NS } & 16 \\ \text { DS } & 2 \\ \text { SWH } & 6172.839 \mathrm{~Hz}\end{array}$

$\begin{array}{ll}\text { SWH } & 6172.839 \mathrm{~Hz} \\ \text { FIDRES } & 0.094190 \mathrm{~Hz}\end{array}$

$A Q \quad 5.3084660 \mathrm{sec}$ 362
81.000 usec 81.000 usec
6.00 usec $299.2 \mathrm{~K}$ $2.00000000 \mathrm{sec}$

$=======$ CHANNEL $f 1======$
NUC1
$1 \mathrm{H}$ $\begin{array}{lr}\text { NUC1 } & 1 \mathrm{H} \\ \mathrm{D} & 9.30 \mathrm{usec}\end{array}$ $\begin{array}{lr}\text { PL1 } & -1.00 \mathrm{~dB} \\ \text { PFO1 } & 300.1318534 \mathrm{MHz}\end{array}$

F2 - Processing parameters SI 32768 $\begin{array}{ll}\mathrm{SF} & 300.1300052 \mathrm{MHz} \\ \text { WDW } & \end{array}$ $\begin{array}{lc}\text { WDW } & E M \\ 5 S B & 0 \\ L B & 0.30 \mathrm{~Hz}\end{array}$ $\begin{array}{lr}G B & 0 \\ P C & 1.00\end{array}$

10 NMP plot parameters

$\begin{array}{ll}\text { CX } & 22.00 \mathrm{~cm} \\ \mathrm{CY} & \end{array}$

$\begin{array}{lr}C Y & 10.00 \mathrm{~cm} \\ F 1 P & 9.000 \mathrm{ppm}\end{array}$

$\begin{array}{ll}F 1 & 2701.17 \mathrm{~Hz} \\ =2 \mathrm{P} & -0.500 \mathrm{pm}\end{array}$

F2 $\quad-150.07 \mathrm{~Hz}$

PPMCM $\quad 0.43182 \mathrm{Dpm} / \mathrm{cm}$

HZCM $\quad 129.60159 \mathrm{~Hz} / \mathrm{cm}$ 
4d- ${ }^{1} \mathrm{H}-\mathrm{NMR}$
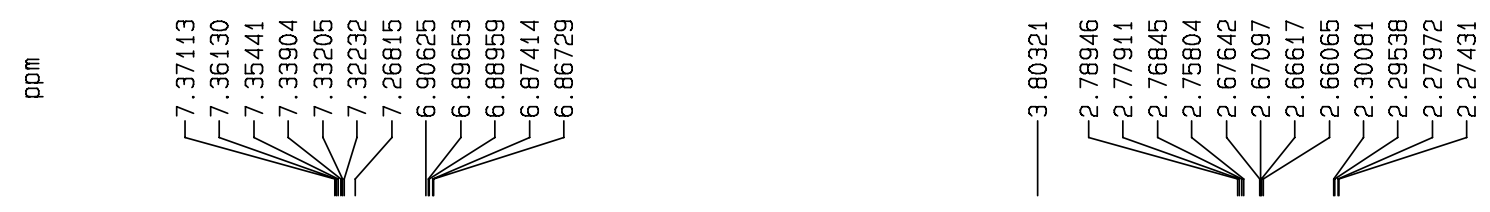

Current Data Parameters

NAME WJY06-0211a

PROCNO

F2 - Acquisition Parameter

Date $\quad 2006021$

Time

16.05

INSTRUM 5 av300

PULPROG Zg

$\begin{array}{ll}\text { TD } & 65536 \\ \text { SOLVNT } & \text { CDC1 }\end{array}$

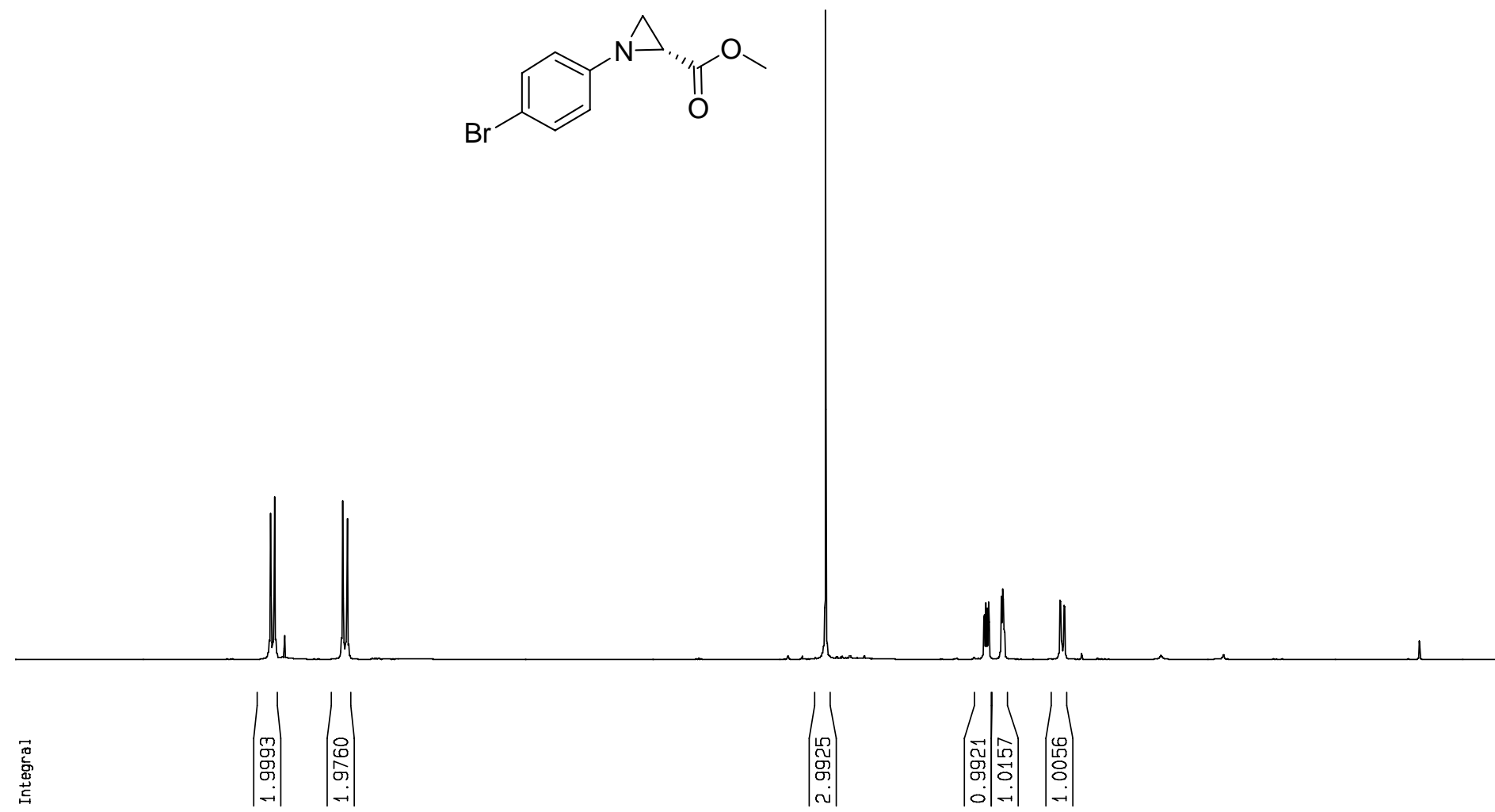

NS

DS

$\begin{array}{lr}\text { SWH } & 2 \\ & 6172.839 \mathrm{~Hz}\end{array}$

AQ $\quad 0.094190 \mathrm{~Hz}$

$\mathrm{AQ} \quad 5.300460 \mathrm{sec}$

$\begin{array}{ll}\text { RG } & 228.1 \\ \text { DW } & 81.000 \text { use }\end{array}$

DE 6.00 usec $297.7 \mathrm{~K}$

D1 $2.00000000 \mathrm{sec}$

$=======$ CHANNEL $f 1=======$
NUC1 $1 \mathrm{H}$ $\begin{array}{lr}\text { NUC1 } & 1 \mathrm{H} \\ \text { P1 } & 9.30 \text { usec }\end{array}$ $\begin{array}{ll}-1.00 \mathrm{~dB} \\ \text { SF01 } & 300.1318534 \mathrm{MHz}\end{array}$

F2 - Processing parameters

SI 32768 SF $\quad 300.1300037 \mathrm{MHz}$ WDW

SSB
LB

GB $E M$
0
$0.30 \mathrm{~Hz}$

10 NMA plot parameters 22.00 $\begin{array}{ll}\text { CX } & 22.00 \mathrm{~cm} \\ \text { CY } & 10.00 \mathrm{~cm}\end{array}$ $\begin{array}{ll}\text { CY } & 10.00 \mathrm{~cm} \\ \text { F1P } & 9.000 \mathrm{ppm}\end{array}$ $\begin{array}{ll}F 1 & 2701.17 \mathrm{~Hz} \\ F 2 \mathrm{P} & -0.500 \mathrm{pPm}\end{array}$ F2 $-150.07 \mathrm{ppm}$ $\begin{array}{ll}\text { PPMCM } & 0.43182 \mathrm{ppm} / \mathrm{cm} \\ \text { HZCM } & 129.60159 \mathrm{~Hz} / \mathrm{cm}\end{array}$ 
4e- ${ }^{1} \mathrm{H}-\mathrm{NMR}$

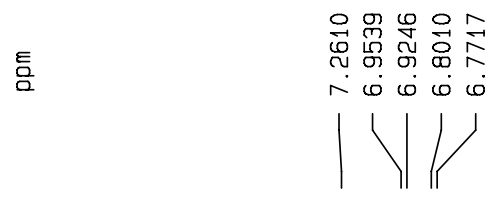

ppm
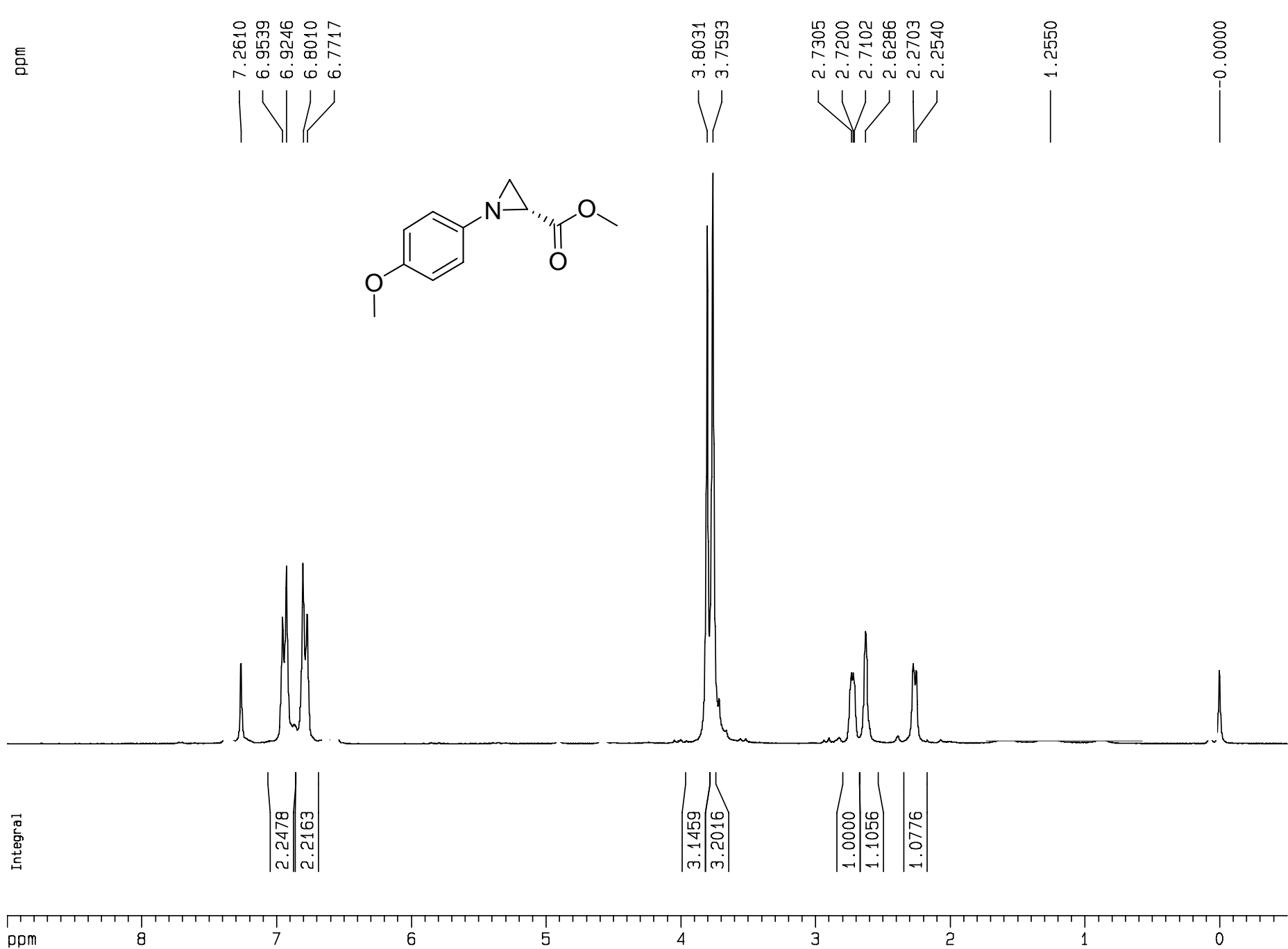

Current Data Parameters

NAME wjy06-0107a

$\begin{array}{ll}\text { EXPNO } & 10 \\ \text { PROCNO } & 1\end{array}$

F2 - Acquisition Parameters

Date 20060107

$\begin{array}{ll}\text { Time } & 16.45 \\ \text { TNSTRUM } & 15300\end{array}$

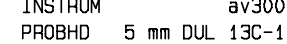

20-1

$\begin{array}{lr}\text { PULPHOG } & 2930 \\ \text { TD } & 65536\end{array}$

SOLVENT CDC13

64
NS

SWH $\quad 6172.839 \mathrm{~Hz}$

IDRES $\quad 0.094190 \mathrm{~Hz}$

AQ $\quad 5.3084660 \mathrm{sec}$

$\begin{array}{lr}\text { RG } & 362 \\ \text { DW } & 81.000 \text { usec } \\ \text { DE } & 6.00 \text { usec }\end{array}$ $2.00000000 \mathrm{sec}$

$=======$ CHANNEL $f 1=======$
NUC1 $1 \mathrm{H}$ $\begin{array}{lr}\text { NUC1 } & 1 \mathrm{H} \\ \mathrm{P} 1 & 9.30 \text { usec }\end{array}$ $\begin{array}{lr}\text { P1 } & \text { 9. } 30 \text { us } \\ \text { PL1 } & -1.00 \mathrm{~dB}\end{array}$ SFO1 $300.1318534 \mathrm{MH}$ F2 - Processing parameters $\begin{array}{cc}\mathrm{SI} & 32768 \\ \mathrm{SF} & 300.1300062 \mathrm{MHz}\end{array}$ WDW EM $\begin{array}{lc}\text { SSB } & 0 \\ \text { LB } & 0.30 \mathrm{~Hz} \\ \text { GB } & 0 \\ \text { PC } & 1.00\end{array}$

10 NMR plot parameters

cX $22.00 \mathrm{~cm}$ $\begin{array}{ll}\text { CY } & 10.00 \mathrm{~cm} \\ \text { F1P } & 9.000 \mathrm{ppm}\end{array}$ F1 $\quad 2701.17 \mathrm{~Hz}$ $\begin{array}{ll}2 \mathrm{p} & -0.500 \mathrm{pp} \\ F_{2} & -150.07 \mathrm{~Hz}\end{array}$ $\begin{array}{ll} & -150.07 \mathrm{~Hz} \\ \text { PPMCM } & 0.43182 \mathrm{pm} / \mathrm{cm}\end{array}$ HZCM $\quad 129.60159 \mathrm{~Hz} / \mathrm{Cm}$ 
4f- ${ }^{1} \mathrm{H}-\mathrm{NMR}$

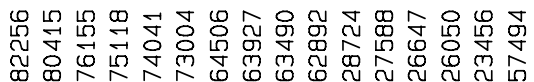
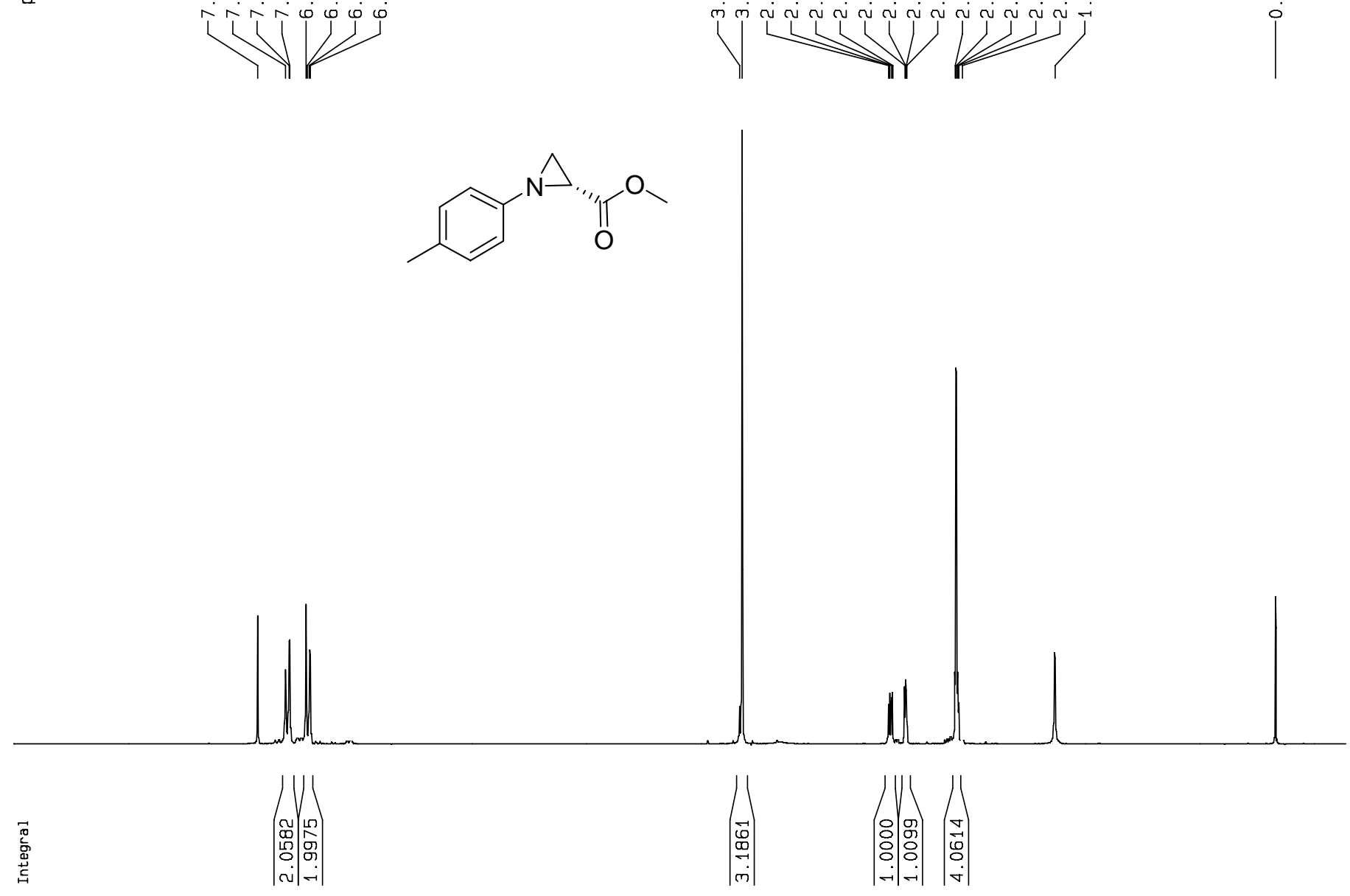

Current Data Parameters

NAME WJY06-0627b

$\begin{array}{lr}\text { EXPNO } & 10 \\ \text { PROCNO } & 1\end{array}$

F2 - Acquisition Parameters 20060627

Time

INSTRUM PROBHD $5 \mathrm{~mm}$ DUL $13 \mathrm{C}-1$

PULPROG

$\begin{array}{ll}\text { TD } & 65536 \\ \text { SOLVENT } & \text { CDC13 }\end{array}$

$\begin{array}{lr}\text { NS } & 10 \\ \text { DS } & 2\end{array}$

SWH $\quad 6172.839 \mathrm{~Hz}$

FIDRES $\quad 0.094190 \mathrm{~Hz}$

$\begin{array}{ll}A Q & 5.3084660 \\ \text { PG } & \end{array}$

DW $\quad 81.000$ usec

DE $\quad 6.00$ usec

D1 $2.00000000 \mathrm{sec}$

$=======$ CHANNEL $f 1======$
NUC1

$\begin{array}{lr}\text { NUC1 } & 1 \mathrm{H} \\ \text { P1 } & 9.30 \text { usec }\end{array}$

$300.1318534 \mathrm{MHz}$

F2 - Processing parameters

$\begin{array}{lc}\text { SI } & 32768 \\ \text { SF } & 300.1300069 \mathrm{MHz}\end{array}$

WOW $\quad$ EM

$\begin{array}{lc}\text { SSB } & 0 \\ \text { LB } & 0.30 \mathrm{~Hz}\end{array}$

$\begin{array}{lr}P B & 0\end{array}$

$\begin{array}{ll}10 \mathrm{NMR} \text { plot parameters } \\ \mathrm{CX} & 22.00 \mathrm{~cm}\end{array}$

$\begin{array}{ll}\text { CY } & 10.00 \mathrm{~cm} \\ \text { F1P } & 9.000 \mathrm{ppm}\end{array}$

$\begin{array}{lr}\text { F1P } & 9.000 \mathrm{ppm} \\ \text { F1 } & 2701.17 \mathrm{~Hz}\end{array}$

$\begin{array}{ll}\text { F1 } & 2701.17 \mathrm{~Hz} \\ \text { F2P } & -0.500 \mathrm{ppm}\end{array}$

$\begin{array}{ll}\text { F2 } & -150.07 \mathrm{~Hz} \\ \text { PPMCM } & 0.43182\end{array}$

$\begin{array}{lr}\text { PPMCM } & 0.43182 \mathrm{ppm} / \mathrm{cm} \\ \mathrm{HZCM} & 129.60159 \mathrm{~Hz} / \mathrm{cm}\end{array}$ 
4g- ${ }^{1} \mathrm{H}-\mathrm{NMR}$

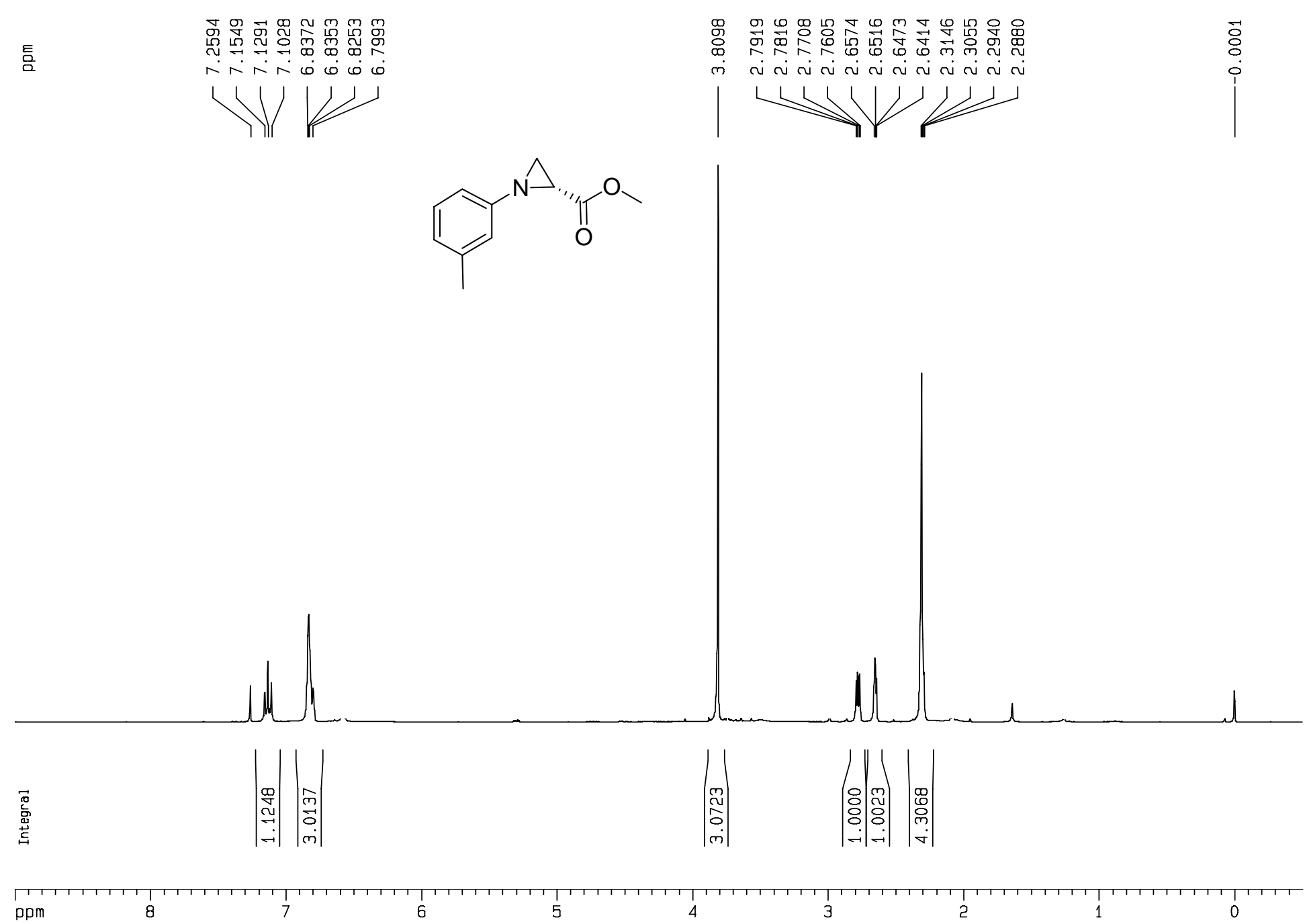

EXPNO

$=2-$ Acquisition Paraneters

Date Acquisition Parameters

Jate $\quad 20060218$

INSTRUM 16.17

PROBHD $5 \mathrm{~mm}$ DUL 13C-1

DULPROG Zg30

$\begin{array}{ll}\text { TD } & 65536 \\ \text { TD } & \text { CDC13 }\end{array}$

$\begin{array}{ll}\text { SWH } & 2 \\ & 6172.839 \mathrm{~Hz}\end{array}$

5. $308190 \mathrm{~Hz}$

AQ $\quad 5.3084660 \mathrm{seC}$

JW $\quad 81.000$ usec

JE $\quad 6.00$ usec

D1 $2.00000000 \mathrm{sec}$

$=======$ CHANNEL $f 1=======$
VUC1 $1 \mathrm{H}$

$\begin{array}{lr}\text { NUC1 } & 1 \mathrm{H} \\ 21 & 9.30 \text { usec }\end{array}$

$\begin{array}{lr}-1.00 \mathrm{~dB} \\ \text { SF01 } & 300.1318534 \mathrm{MHz}\end{array}$

$=2$ - Processing parameters

I Processing parameters

SF $\quad 300.1300064 \mathrm{MHz}$

WOW

SSB
LB

GB

EM
0
$0.30 \mathrm{~Hz}$

1.00

10 NMR plot parameters

$\begin{array}{ll}\text { CX } & 22.00 \mathrm{~cm} \\ \mathrm{CY} & 9.000 \mathrm{~cm}\end{array}$

$\begin{array}{ll}\mathrm{CY} & 10.00 \mathrm{~cm}\end{array}$

$\begin{array}{ll}F 1 & 9.000 \mathrm{ppm} \\ F & 2701.17 \mathrm{~Hz}\end{array}$

$\begin{array}{ll}-1 & -0.500 \mathrm{ppm} \\ =2 & -150.07 \mathrm{~Hz}\end{array}$

$\begin{array}{lr}\text { PPMCM } & 0.43182 \mathrm{ppm} / \mathrm{cm} \\ \text { HZCM } & 129.60159 \mathrm{~Hz} / \mathrm{cm}\end{array}$

ppm 
4h- ${ }^{1} \mathrm{H}-\mathrm{NMR}$

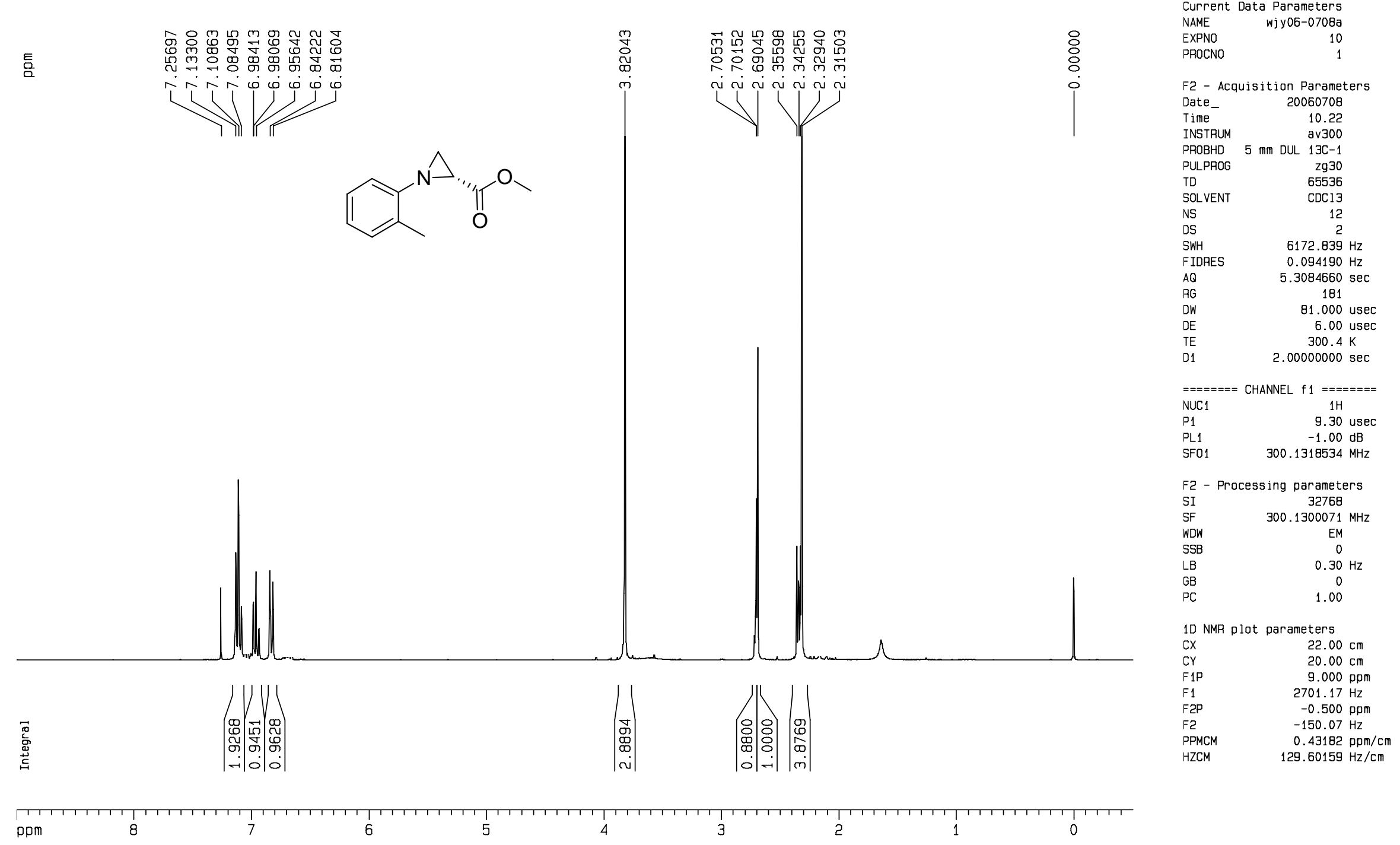


4h- ${ }^{13} \mathrm{C}-\mathrm{NMR}$

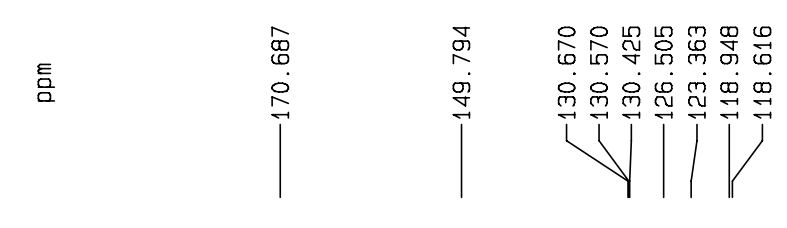

\section{Hin}

ppm

$\int_{160}$

140

120

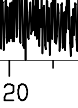

iniming

100
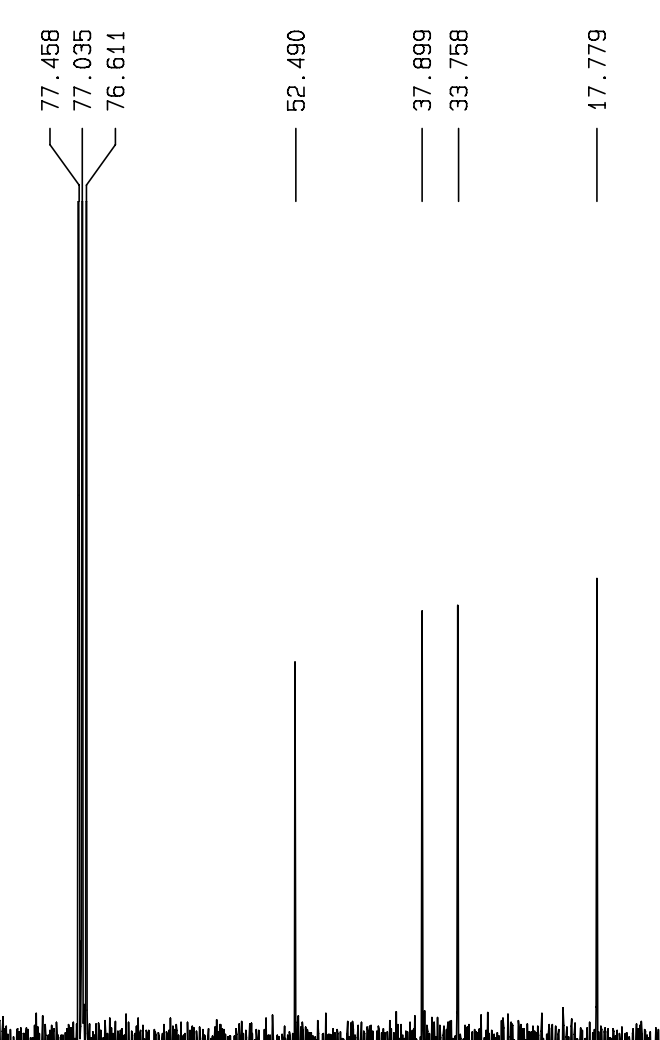

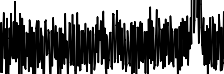

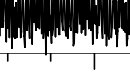

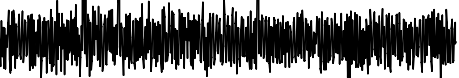

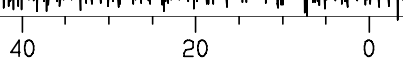

60

40

20

0 
10+11- ${ }^{1} \mathrm{H}$ NMR

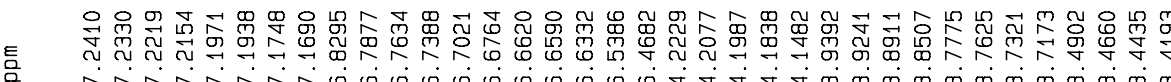
nnn (1)

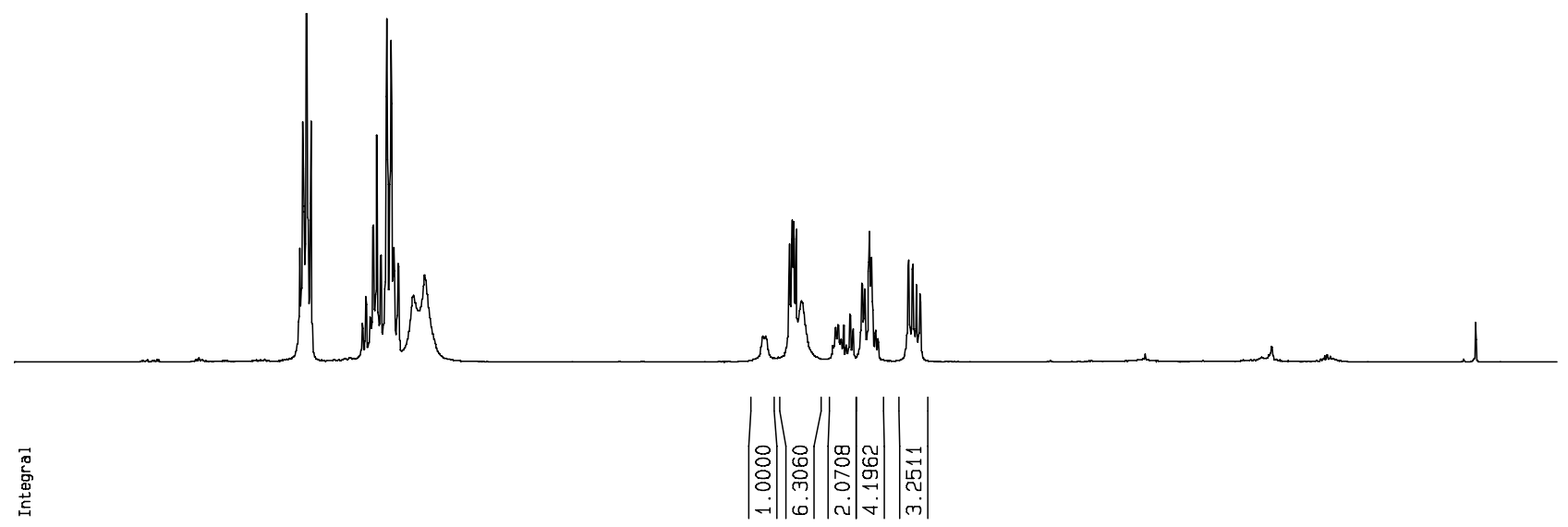

Current Data Parameters

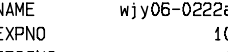
PROCNO

F2 - Acquisition Parameters

$\begin{array}{ll}\text { Date } & 20060222 \\ \text { Time } & 15.09\end{array}$ INSTRUM PROBHD $5 \mathrm{~mm}$ DUL $13 \mathrm{C}-1$ PULPROG $\quad 2930$ SOLVENT NS

$\begin{array}{lc}\text { NS } & 16 \\ \text { DS } & 2 \\ \text { SWH } & 6172.839 \mathrm{~Hz}\end{array}$

$\begin{array}{ll}0.094190 \mathrm{~Hz} \\ \mathrm{AQ} & 5.3084660 \mathrm{sBCS}\end{array}$

$\begin{array}{rr}A Q & 5.3084660 \mathrm{sec} \\ \mathrm{AG} & 114 \\ \mathrm{DW} & 81.000 \text { use }\end{array}$

81.000 usec
DE $296.7 \mathrm{~K}$
$.00000000 \mathrm{sec}$

$=======$ CHANNEL $f 1=======$
NUC1 $1 \mathrm{H}$

9.30 usec

$\begin{array}{ll}-1.00 \mathrm{~dB} \\ \mathrm{SF} 01 & 300.1318534 \mathrm{MHz}\end{array}$

F2 - Processing parameters

SI 32768

WDW $\quad 300.1300116$

$\begin{array}{cc}\text { SSB } & 0 \\ \text { LB } & 0.30 \mathrm{~Hz} \\ G B & 0\end{array}$

10 NMA plot parameters

$\begin{array}{ll}\text { CX } & \\ \mathrm{CY} & 22.00 \mathrm{~cm}\end{array}$

$\begin{array}{lr}\text { CY } & 5.00 \mathrm{~cm} \\ F 1 \mathrm{P} & 9.000 \mathrm{ppm}\end{array}$

$\begin{array}{ll}11 & 2701.17 \mathrm{~Hz} \\ =2 \mathrm{P} & -0.500\end{array}$

$\begin{array}{ll}F 2 & -150.07 \mathrm{~Hz} \\ \text { PPMCM } & 0.43182 \mathrm{pz}\end{array}$

$\begin{array}{lr}\text { PPMCM } & 0.43182 \mathrm{ppm} / \mathrm{cm} \\ \text { HZCM } & 129.60159 \mathrm{~Hz} / \mathrm{cm}\end{array}$

ppm

3

0 
$10+11-{ }^{13} \mathrm{C}$ NMR

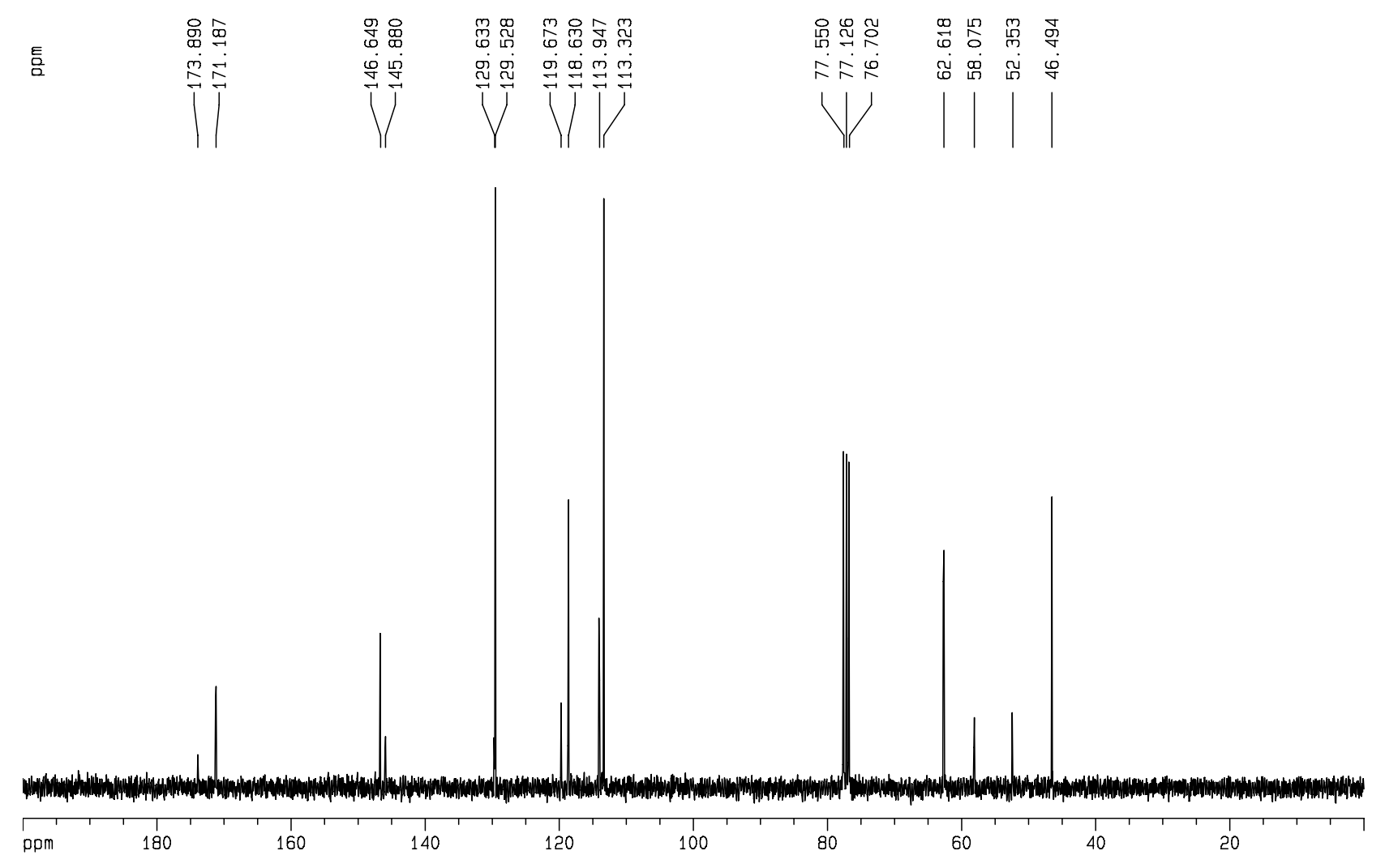

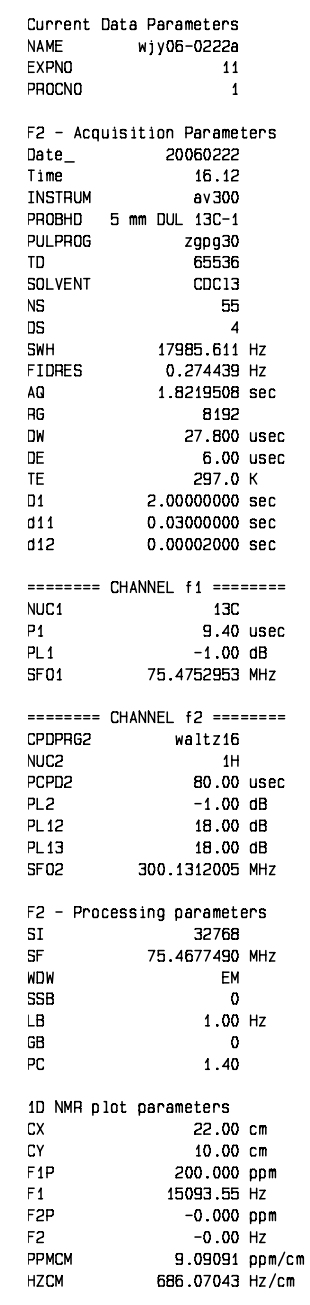


13+14- ${ }^{1} \mathrm{H}$ NMR

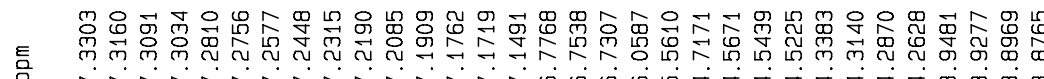

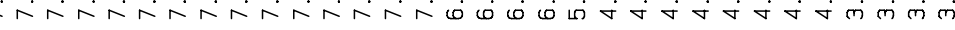
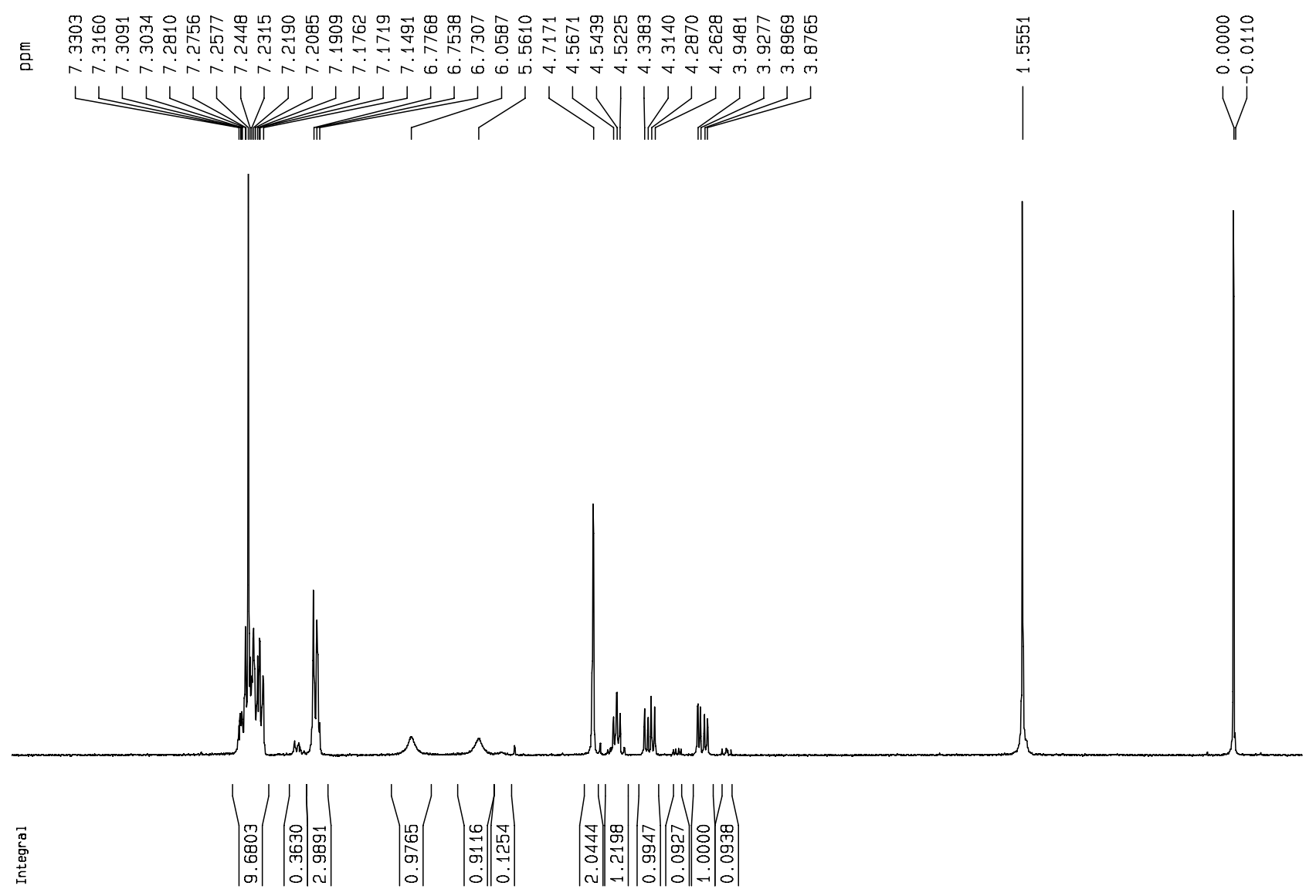

Current Data Parameters

XXPNO WiY06-0710a

PROCNO

F2 - Acquisition Parameters

$\begin{array}{ll}\text { Date } & 20060710 \\ \text { Time } & 15.17\end{array}$

$\begin{array}{ll}16.17 \\ \text { INSTRUM } & \text { av300 }\end{array}$

$5 \mathrm{~mm}$ DUL $13 \mathrm{C}-1$

$\begin{array}{lr}\text { PULPROG } & 2930 \\ \text { TD } & 65536 \\ \text { SOLVNT } & \text { CDC13 }\end{array}$

NS

$\begin{array}{lr} & \\ \text { DS } & 2 \\ \text { SWH } & 6172.839 \mathrm{HZ}\end{array}$

$\begin{array}{ll}\text { SWH } & 6172.839 \mathrm{~Hz} \\ \text { FIDRES } & 0.094190 \mathrm{~Hz}\end{array}$

$\begin{array}{ll}A Q & 5.3084660 \mathrm{sec} \\ \mathrm{AG} & 287.4\end{array}$

DW $\quad 81.000$ usec

DE $\quad 6.00$ usec

$2.00000000 \mathrm{k}$

$=======$ CHANNEL $f 1=======$
NUC1
PH

19.30 usec

$\begin{array}{ll}-1.00 \mathrm{~dB} \\ \mathrm{SF} 01 & 300.1318534 \mathrm{MHZ}\end{array}$

F2 - Processing parameters

SI $\quad 32768$

$5 \mathrm{~F}$
WOW

$\begin{array}{lc}\text { WOW } & E M \\ \text { SSB } & 0 \\ \text { LB } & 0.30 \mathrm{~Hz} \\ \text { GB } & 0\end{array}$

10 NMA plot parameters

$\begin{array}{ll}\text { CX } & 22.00 \mathrm{~cm} \\ \mathrm{CY} & \end{array}$

$\begin{array}{ll} & \\ \mathrm{CY} & 10.00 \mathrm{~cm}\end{array}$

$=1 \quad 2701.17 \mathrm{~Hz}$

$=2-150.07 \mathrm{~Hz}$

PPMCM $\quad 0.43182 \mathrm{ppm} / \mathrm{Cm}$

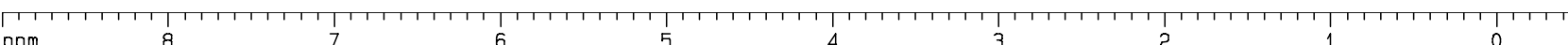


13+14- ${ }^{13} \mathrm{C}$ NMR

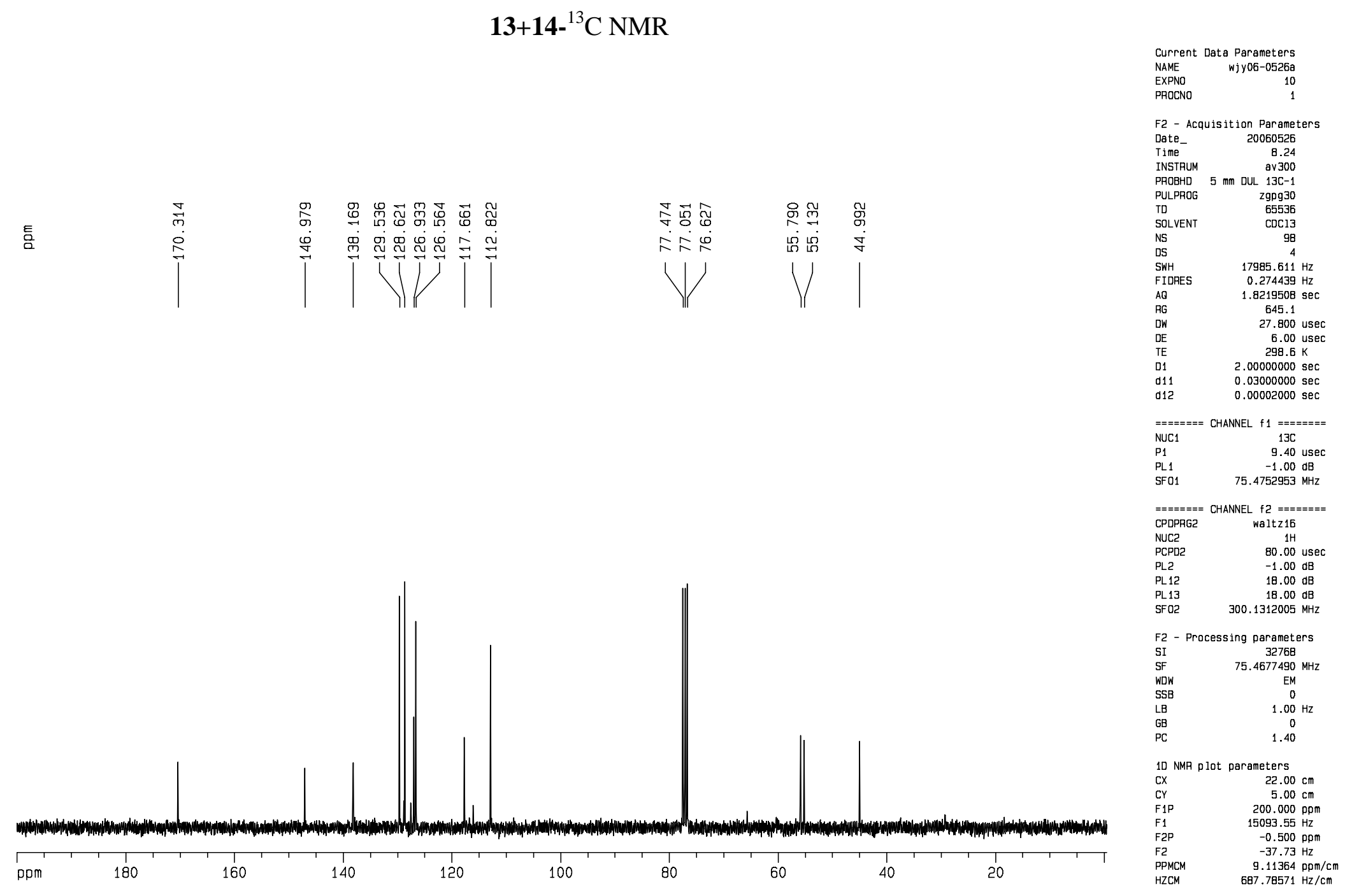


15a- ${ }^{1} \mathrm{H}-\mathrm{NMR}$

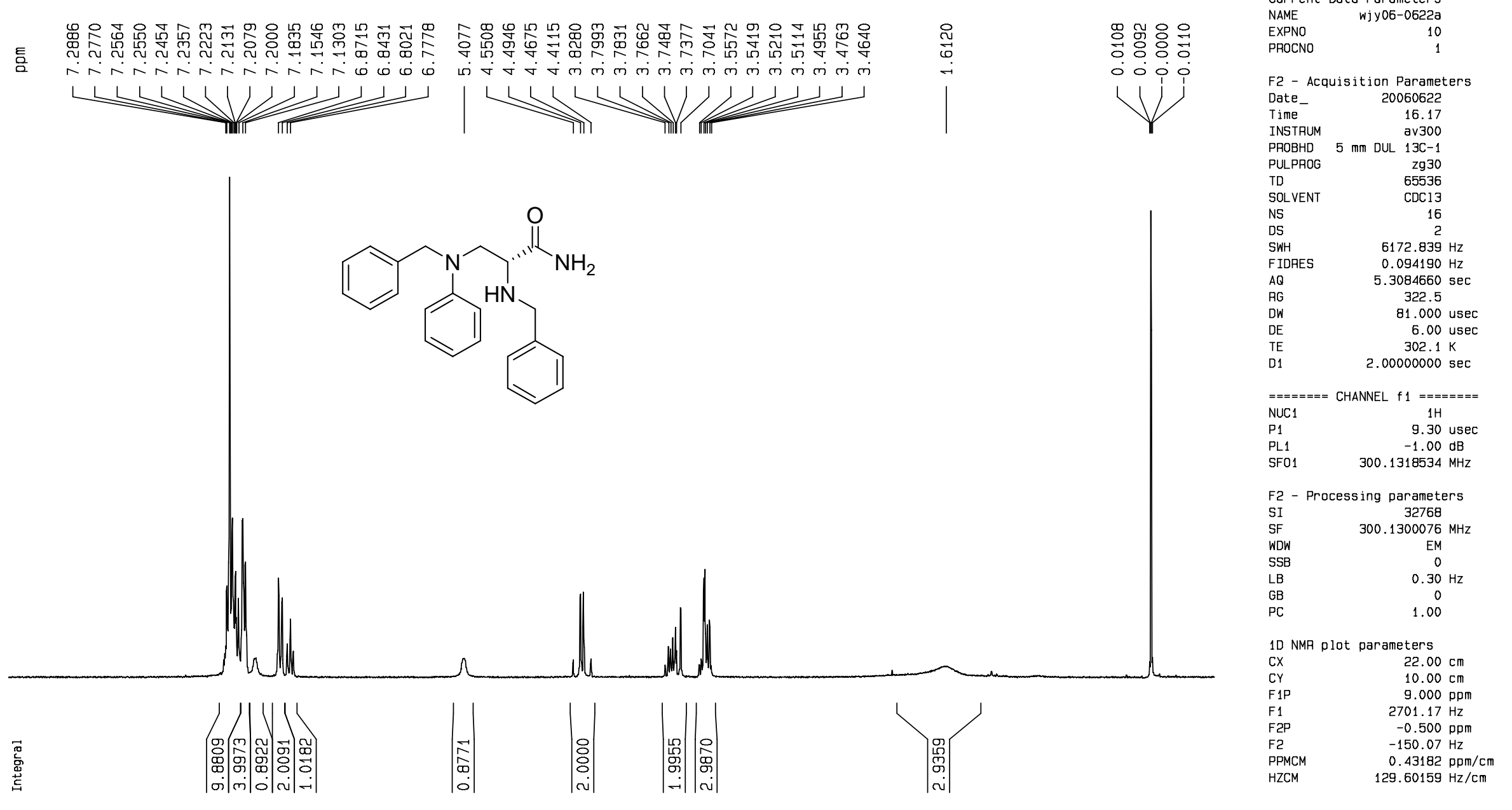


15a- ${ }^{13} \mathrm{C}-\mathrm{NMR}$

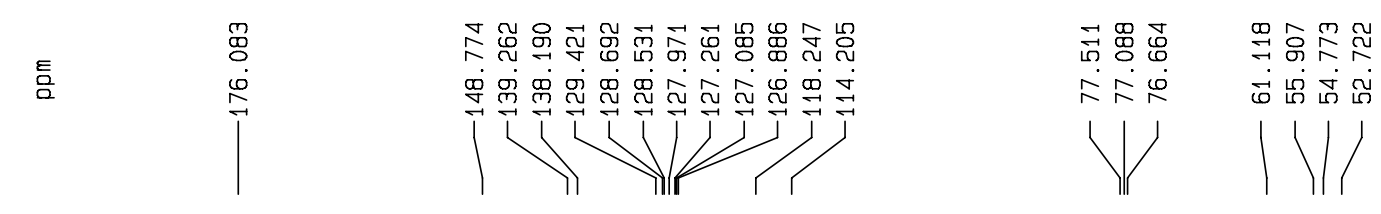

Current Data Parameters

NAME
EXPNO

PROCNO

F2 - Acquisition Parameters

Date

Time
INSTRUM

PULPROG zgpg30

$\begin{array}{ll}\text { TD } & 65536 \\ \text { SOLVENT } & \text { CDC13 }\end{array}$

NS
DS

SWH $\quad 47985.611 \mathrm{~Hz}$

FIDRES $\quad 0.274439 \mathrm{~Hz}$

$\begin{array}{lc}\mathrm{AQ} & 1.8219508 \mathrm{sec} \\ \mathrm{AG} & 812.7\end{array}$

$\begin{array}{rr}\text { RW } & 87.800 \text { usec } \\ \text { DE } & 5.00 \text { usec }\end{array}$

$\begin{array}{lr}\text { TE } & 301.0 \mathrm{~K} \\ \text { D1 } & 2.00000000 \mathrm{sec}\end{array}$

$\begin{array}{ll}01 & 2.00000000 \mathrm{sec} \\ 011 & 0.03000000 \mathrm{sec} \\ 012 & 0.00002000 \mathrm{sec}\end{array}$

$=======$ CHANNEL $f 1$ ==s====

$\begin{array}{lr}\text { NUC1 } & 13 \mathrm{C} \\ \text { P1 } & 9.40 \text { usec } \\ \text { PL1 } & -1.00 \mathrm{~dB}\end{array}$

$-1.00 \mathrm{~dB}$
$\quad 75.4752953 \mathrm{MHZ}$

$=======$ CHANNEL f $2==== \pm==$
CPDPRG2
waltz16

$\begin{array}{lc}\text { NUC2 } & 1 \mathrm{H} \\ \text { PCPO2 } & 80.00 \text { use }\end{array}$

$\begin{array}{ll}\text { PCPD2 } & 80.00 \mathrm{usec} \\ \text { PL2 } & -1.00 \mathrm{~dB} \\ \text { PL12 } & 18.00 \mathrm{~dB} \\ \text { L11 } & 1.00 \mathrm{~d}\end{array}$

$\begin{array}{ll}\text { PL12 } & 18.00 \mathrm{~dB} \\ \text { PL13 } & 18.00 \mathrm{~dB}\end{array}$

SF $02 \quad 300.1312005 \mathrm{MHZ}$

F2 - Processing parameters

$\begin{array}{lc}\text { SI } & 32768 \\ \text { SF } & 75.4677490 \mathrm{MHZ}\end{array}$

$\begin{array}{lc}\text { WDW } & \text { EM } \\ \text { SSB } & 0 \\ \text { LB } & 1.00 \mathrm{HZ}\end{array}$

$\begin{array}{lr}\text { GB } & 0 \\ \text { PC } & 1.40\end{array}$

10 NMR p lot parameters

$\begin{array}{lr}C X & 22.00 \mathrm{~cm} \\ \mathrm{CY} & 5.00 \mathrm{~cm}\end{array}$

$\begin{array}{lr}\text { F1P } & 200.000 \mathrm{ppm} \\ \text { F1 } & 15093.55 \mathrm{~Hz}\end{array}$

$\begin{array}{ll}-5.000 \mathrm{pp} \\ \mathrm{F} & -377.34 \mathrm{~Hz}\end{array}$

$\begin{array}{lr}\text { F2 } & -377.34 \mathrm{~Hz} \\ \text { PPMCM } & 9.31818 \mathrm{ppm} / \mathrm{cm}\end{array}$ 
15b- ${ }^{1} \mathrm{H}-\mathrm{NMR}$

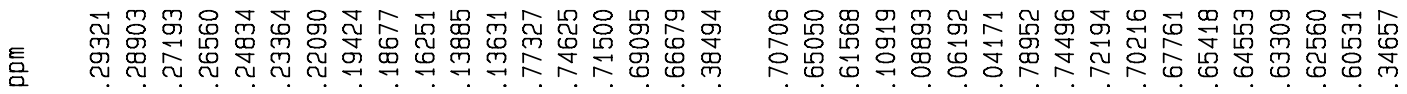

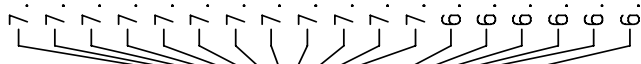
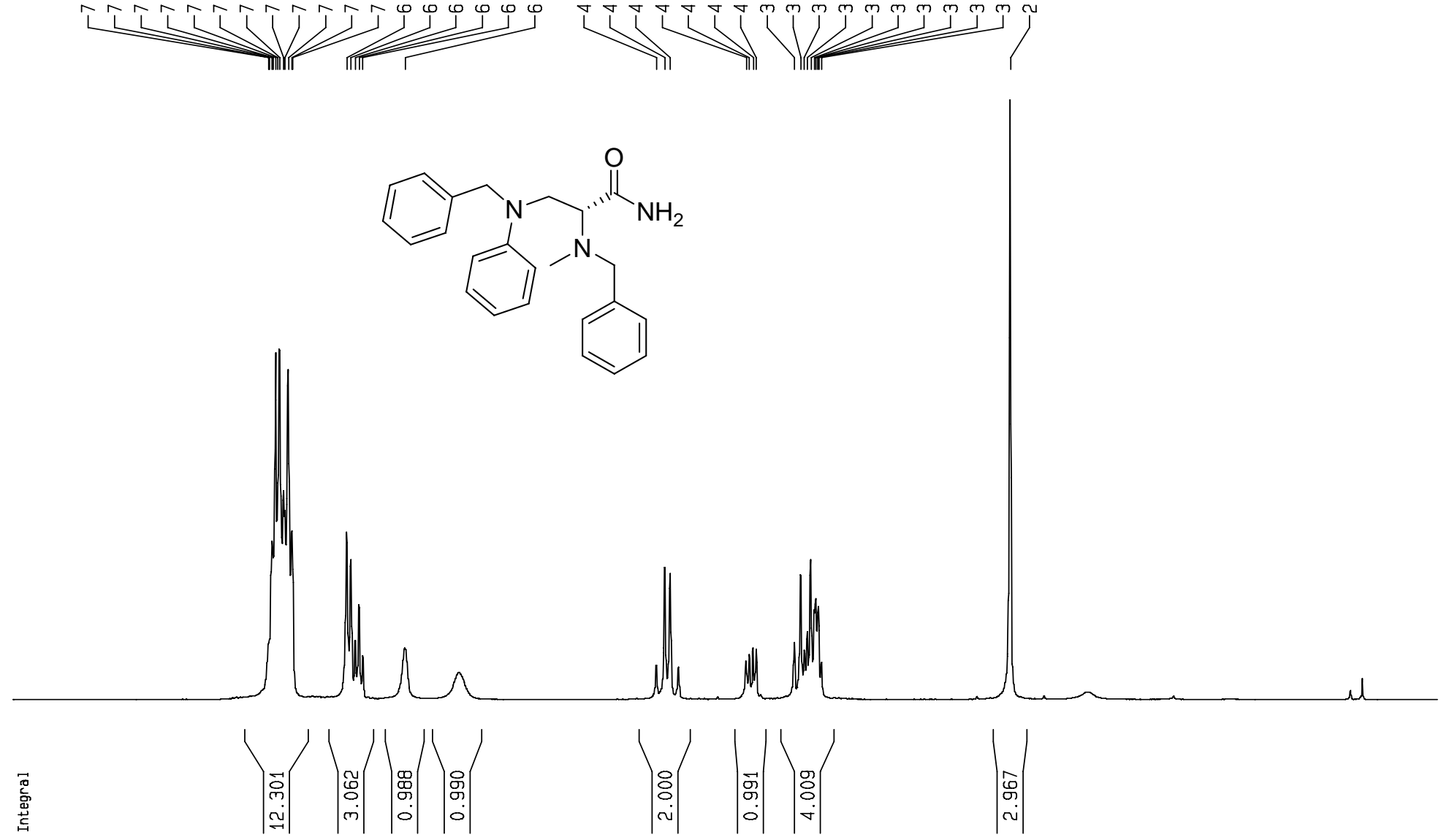

pm 8
6
Current Data Parameters NAME WJY06-0708 PROCNO

F2 - Acquisition Parameters

Jate 20060708

Time

INSTRUM av300

PULPHOG $5 \mathrm{~mm}$ DUL

$\begin{array}{lr}\text { PULPROG } & 2930 \\ \text { TD } & 65536\end{array}$

SOLVENT

JS

$\begin{array}{lr}\text { SWH } & \text { 2 } \\ \text { FID } & 6172.839 \mathrm{~Hz}\end{array}$

TIDRES $\quad 0.094190 \mathrm{~Hz}$

$A Q-5.30860 \mathrm{sec}$

DW $\quad 81.000$ usec

$\begin{array}{ll}\mathrm{TE} & 6.00 \mathrm{usec} \\ \mathrm{TE} & 300.4 \mathrm{~K}\end{array}$

$012.00000000 \mathrm{sec}$

$=======$ CHANNEL $f 1=======$
NUC1 $1 \mathrm{H}$

$\begin{array}{lr}\text { NUC1 } & 1 \mathrm{H} \\ \mathrm{P} 1 & 9.30 \text { use }\end{array}$

$\begin{array}{lr}\text { PL1 } & \text { - } \\ \text { PL1 } & -1.00 \mathrm{~dB} \\ \text { SFO1 } & 300.1318534 \mathrm{MHZ}\end{array}$

F2 - Processing parameters

SI Processing parameters

SF $\quad 300.1300224 \mathrm{MHz}$

WDW

LB

${ }^{G B}$

EM

0
$0.30 \mathrm{~Hz}$
0

10 NMR plot parameters

$\begin{array}{ll}\text { CX } & \text { CY.00 cm } \\ C Y & 10.00\end{array}$

$\begin{array}{ll}\mathrm{CY} & 10.00 \mathrm{~cm} \\ F 1 \mathrm{P} & 9.000 \mathrm{ppm} \\ -1 & -0.500 \mathrm{pz}\end{array}$

$\begin{array}{ll}=1 & 2701.17 \mathrm{~Hz} \\ = & -15007 \mathrm{ppm}\end{array}$

$\begin{array}{ll}72 \mathrm{P} & -0.500 \mathrm{ppm} \\ 72 & -150.07 \mathrm{~Hz}\end{array}$

$\begin{array}{lr}\text { PPMCM } & 0.43182 \mathrm{ppm} / \mathrm{cm} \\ \text { HZCM } & 129.60161 \mathrm{~Hz} / \mathrm{cm}\end{array}$ 
15b- ${ }^{13} \mathrm{C}-\mathrm{NMR}$
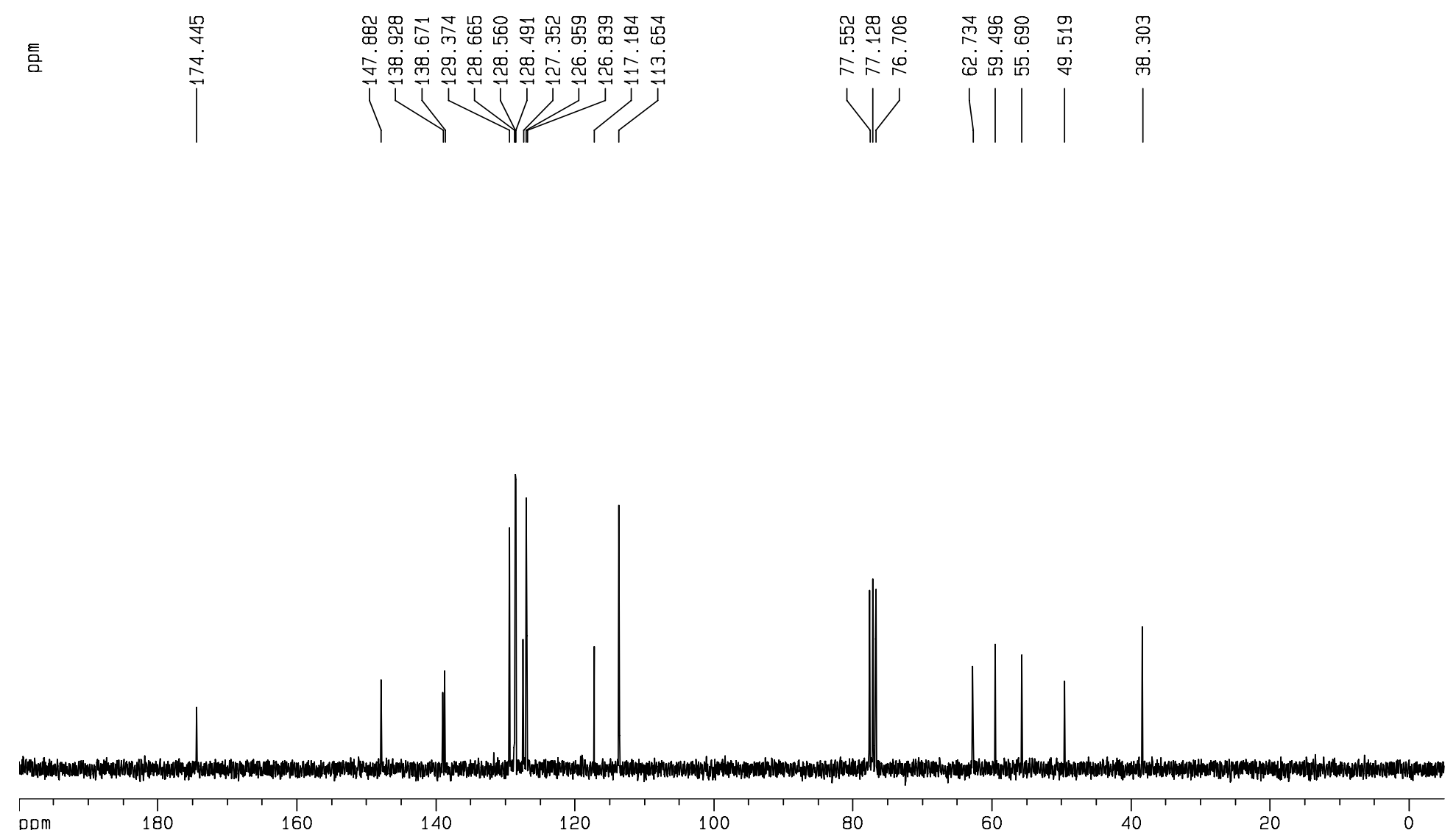

Current Data Parameters

wjyo6-0708

EXPNO
PROCNO

F2- Acquisition Parameters
Jate_ 20060708
Time

Time

11.04

$\begin{array}{lr}\text { PULPROG } & \text { 2gpg30 } \\ \text { TD } & 65536\end{array}$

$\begin{array}{lr}\text { SOLVENT } & \text { CDC13 } \\ \text { NS } & 26 \\ \text { JS } & 4\end{array}$

$\begin{array}{lr}\text { SWH } & 17985.611 \mathrm{~Hz} \\ \text { FIDRES } & 0.274439 \mathrm{~Hz}\end{array}$

FIDRES $\quad 0.274439 \mathrm{~Hz}$

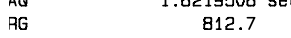

$\begin{array}{rr}\mathrm{JW} & 27.800 \text { usec } \\ \mathrm{JE} & 5.00 \mathrm{usec}\end{array}$

$2.0000000 \mathrm{~K}$

$\begin{array}{ll}011 & 0.03000000 \mathrm{sec}\end{array}$

d12 $0.00002000 \mathrm{sec}$

$=======$ CHANNEL $f 1$ $======$

$\begin{array}{ll}\text { NUC1 } & 13 \mathrm{C} \\ \mathrm{P}_{1} & 9.40 \mathrm{usec} \\ \mathrm{DL} & -1.00 \mathrm{~dB}\end{array}$

$\begin{array}{lr}\text { PL1 } & -9.40 \mathrm{usec} \\ \text { SF01 } & 75.4752953 \mathrm{MHz}\end{array}$

$=======$ CHANNEL $\mathrm{f} 2=======$
waltz16

$\begin{array}{lr}\text { CPOPRG } & \text { waltz16 } \\ \text { NUC2 } & 1 \mathrm{H}\end{array}$

$\begin{array}{ll}\text { PCPD2 } & 80.00 \text { us } \\ \text { PL2 } & -1.00 \mathrm{~dB} \\ \text { PL12 } & 18.00 \mathrm{~dB}\end{array}$

PL13 $\quad 18.00 \mathrm{~dB}$

SF02 $300.1312005 \mathrm{MHZ}$

F2 - Processing parameters

$\begin{array}{ll}\text { SI } & 32768 \\ \mathrm{SF} & 75.4677490 \mathrm{MHZ}\end{array}$

$\begin{array}{lc}\text { WOW } & \text { EM } \\ \text { SSB } & 0 \\ \text { B } & 1.00 \mathrm{~Hz}\end{array}$

$\begin{array}{lr}\text { GB } & 0 \\ \text { PC } & 1.40\end{array}$

10 NMR plot parameters

$\begin{array}{rr}c X & 22.00 \mathrm{~cm} \\ \mathrm{cY} & 5.00 \mathrm{~cm}\end{array}$

$\begin{array}{lr}\text { CY } & 5.00 \mathrm{~cm} \\ \text { F1P } & 200.000 \mathrm{ppm} \\ =1 & 15093.55 \mathrm{~Hz}\end{array}$

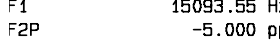

F2 $\quad-377.34 \mathrm{~Hz}$

$\begin{array}{ll}\text { HZCM } & 9.31818 \mathrm{ppm} / \mathrm{cm} \\ 703.22217 \mathrm{~Hz} / \mathrm{cm}\end{array}$ 
15c- ${ }^{1} \mathrm{H}-\mathrm{NMR}$

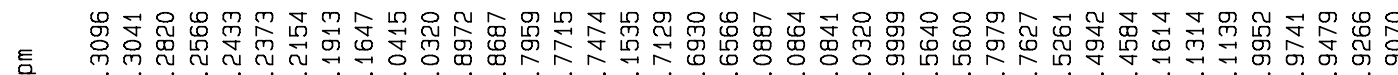

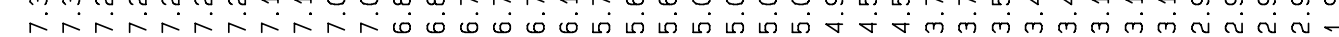

(L)
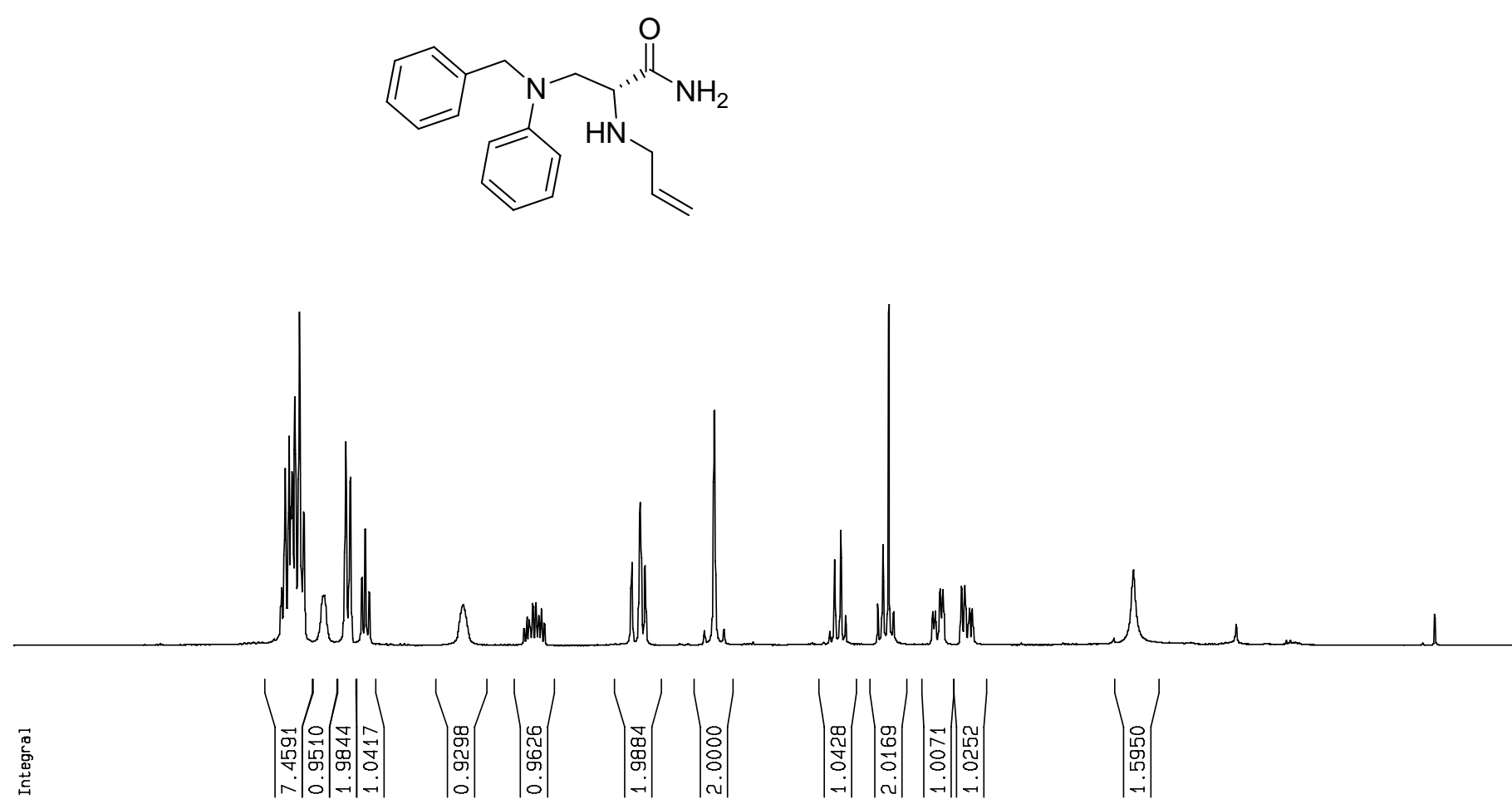

urrent Data Parameters

NAME WJy06-0714a

PROCNO

F2 - Acquisition Parameters

Jate $\quad 20060714$

16.16
INSTRUM
av300

PROBHO $5 \mathrm{~mm}$ DUL $13 \mathrm{C}-1$

\begin{tabular}{lr} 
PULPROG & 2930 \\
TD & 65536 \\
\hline
\end{tabular}

$\begin{array}{lll}\mathrm{CDC} 13 \\ \text { SOLVENT } & 11\end{array}$

$\begin{array}{lr}\text { JS } & 2 \\ \text { SWH } & 6172.839 \mathrm{~Hz}\end{array}$

IDRES $\quad 0.094190 \mathrm{~Hz}$

$\begin{array}{ll}A Q & 5.3084660 \mathrm{sec}\end{array}$

$\begin{array}{lr}\text { TW } & 90.5 \\ \text { DW } & 81.000 \text { usec }\end{array}$ 6.00 usec $2.00000000 \mathrm{sec}$

$=======$ CHANNEL $f 1=======$
VUC1 $\begin{array}{lr}\text { NUC1 } & 1 \mathrm{H} \\ \mathrm{P}_{1} & 9.30 \mathrm{usec}\end{array}$

PL1 $\quad-1.00 \mathrm{~dB}$

F2 - Processing parameters

SI 32768

$\quad 300.1300131 \mathrm{MHz}$

$\begin{array}{lr}\text { WDW } & \text { EM } \\ \text { SSB } & 0\end{array}$

$\begin{array}{cc}L B & 0.30 \mathrm{~Hz} \\ G B & 0 \\ O C & 1.00\end{array}$

10 NMF plot parameters

$\begin{array}{ll}C X & 22.00 \mathrm{~cm} \\ \mathrm{CY} & 5.00 \mathrm{~cm}\end{array}$

$\begin{array}{lr}\text { CY } & 5.00 \mathrm{~cm} \\ \text { F1P } & 9.000 \mathrm{ppm} \\ -1 & 27.17 \mathrm{mz}\end{array}$

$\begin{array}{ll}=1 & 2701.17 \mathrm{~Hz} \\ = & -00.500 \mathrm{pHz}\end{array}$

$\begin{array}{ll}-2 \mathrm{p} & -0.500 \mathrm{ppm} \\ =2 & -150.07 \mathrm{~Hz}\end{array}$

PPMCM $\quad 0.43182 \mathrm{ppm} / \mathrm{c}$

HZCM $\quad 129.60159 \mathrm{~Hz} / \mathrm{cm}$ 
15c- ${ }^{13} \mathrm{C}-\mathrm{NMR}$
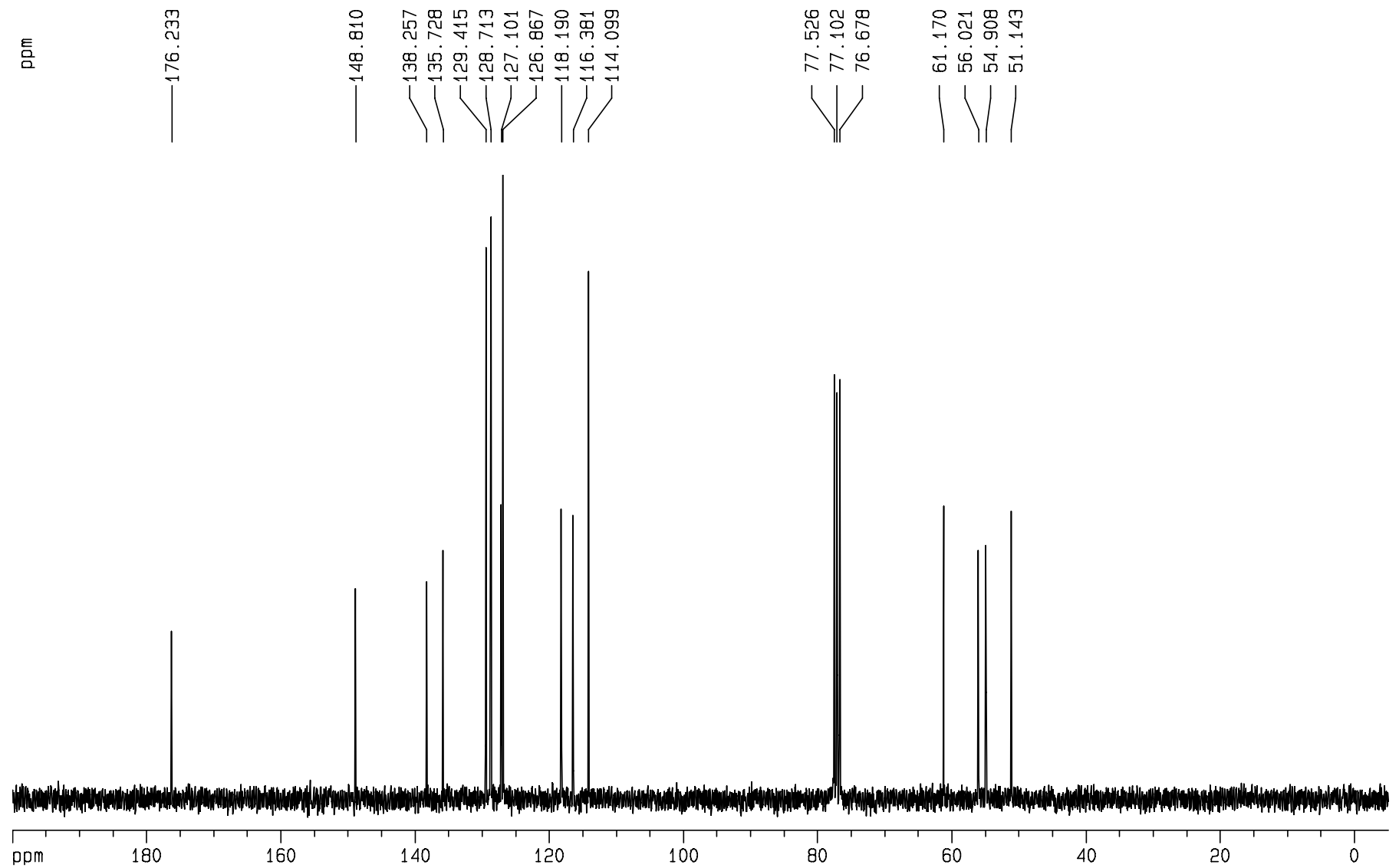

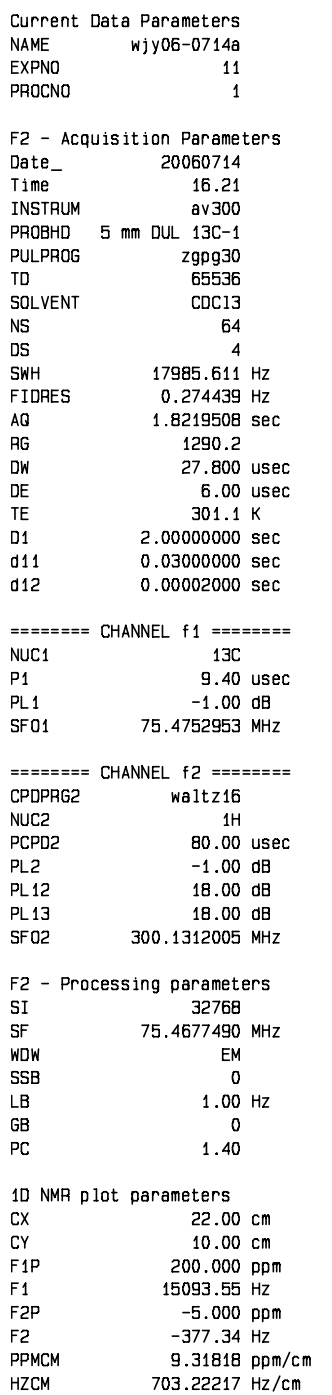


15d- ${ }^{1} \mathrm{H}-\mathrm{NMR}$

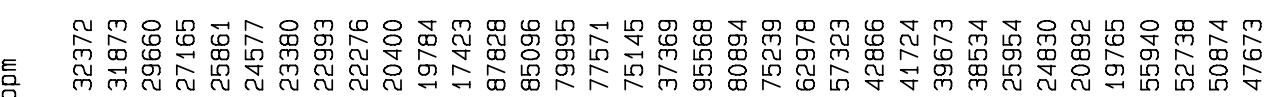
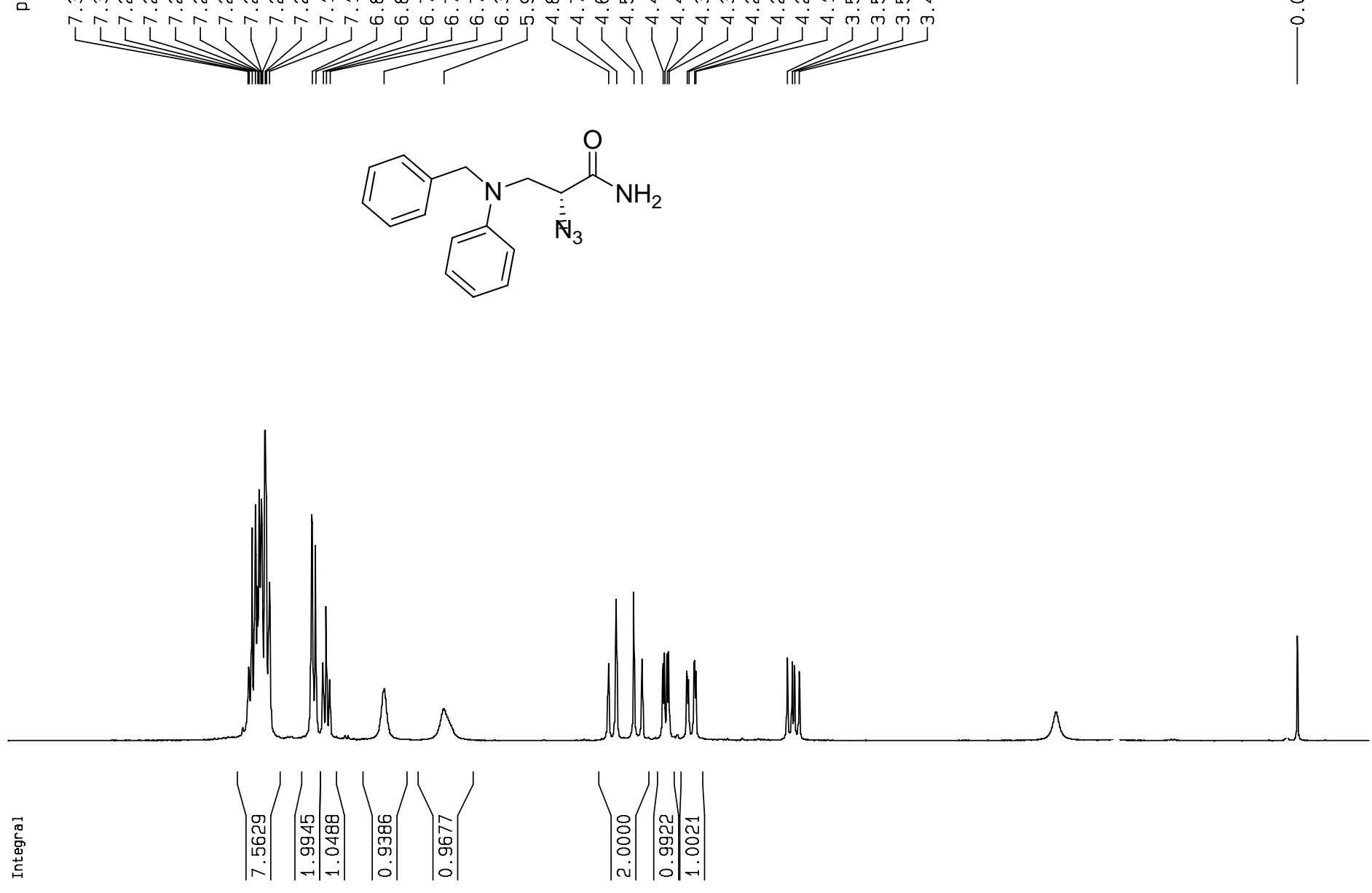

Current Data Parameters

NAME wjy06-0708b

EXPNO

2 - Acquisition Parameters

$-5-A c a$

Date $\quad 20060708$

Time
INSTRUM

30300

PROBHD $5 \mathrm{~mm}$ DUL $13 \mathrm{C}-1$

PULPROG Zg30

$\begin{array}{ll}\text { TD } & 65536 \\ \text { SOLNT } & \text { CDC13 }\end{array}$

$\begin{array}{lr}\text { SOLVENT } & \text { CDC13 } \\ \text { NS } & 16\end{array}$

$\begin{array}{lr}\text { DS } & 2 \\ \text { SWH } & 6172.839 \mathrm{~Hz}\end{array}$

IDRES $\quad 0.094190 \mathrm{~Hz}$

$A Q \quad 5.3084660$

$\begin{array}{ll}7 G & 203.2 \\ \text { JW } & 81.000\end{array}$

81.000 usec
6.00 usec

6.00 usec
$300.4 \mathrm{~K}$

$012.00000000 \mathrm{sec}$

$=======$ CHANNEL $f 1$
NUC1 $=======$

P1 9.30 usec

$-1.00 \mathrm{~dB}$

- Processing parameters

SI 32768

WDW

WDW
$5 S B$

$\begin{array}{ll}S S B & 0.30 \mathrm{~Hz}\end{array}$

PC $\quad 1.00$

10 NMR plot parameters

$\begin{array}{lr}C X & 22.00 \mathrm{~cm} \\ C Y & 5.00 \mathrm{~cm}\end{array}$

$\begin{array}{lc}\text { CY } & 5.00 \mathrm{~cm} \\ =1 \mathrm{P} & 9.000 \mathrm{ppm}\end{array}$

$\begin{array}{lr}=1 & 9.000 \mathrm{ppm} \\ =1 & 2701.17 \mathrm{~Hz}\end{array}$

$\begin{array}{ll}\text { F2P } & -0.500 \mathrm{ppm} \\ =2 & -150.07 \mathrm{~Hz}\end{array}$

$\begin{array}{ll} & -150.07 \mathrm{~Hz} \\ \text { PPMCM } & 0.43182 \mathrm{ppm} / \mathrm{cm}\end{array}$

? 
15d- ${ }^{13} \mathrm{C}-\mathrm{NMR}$
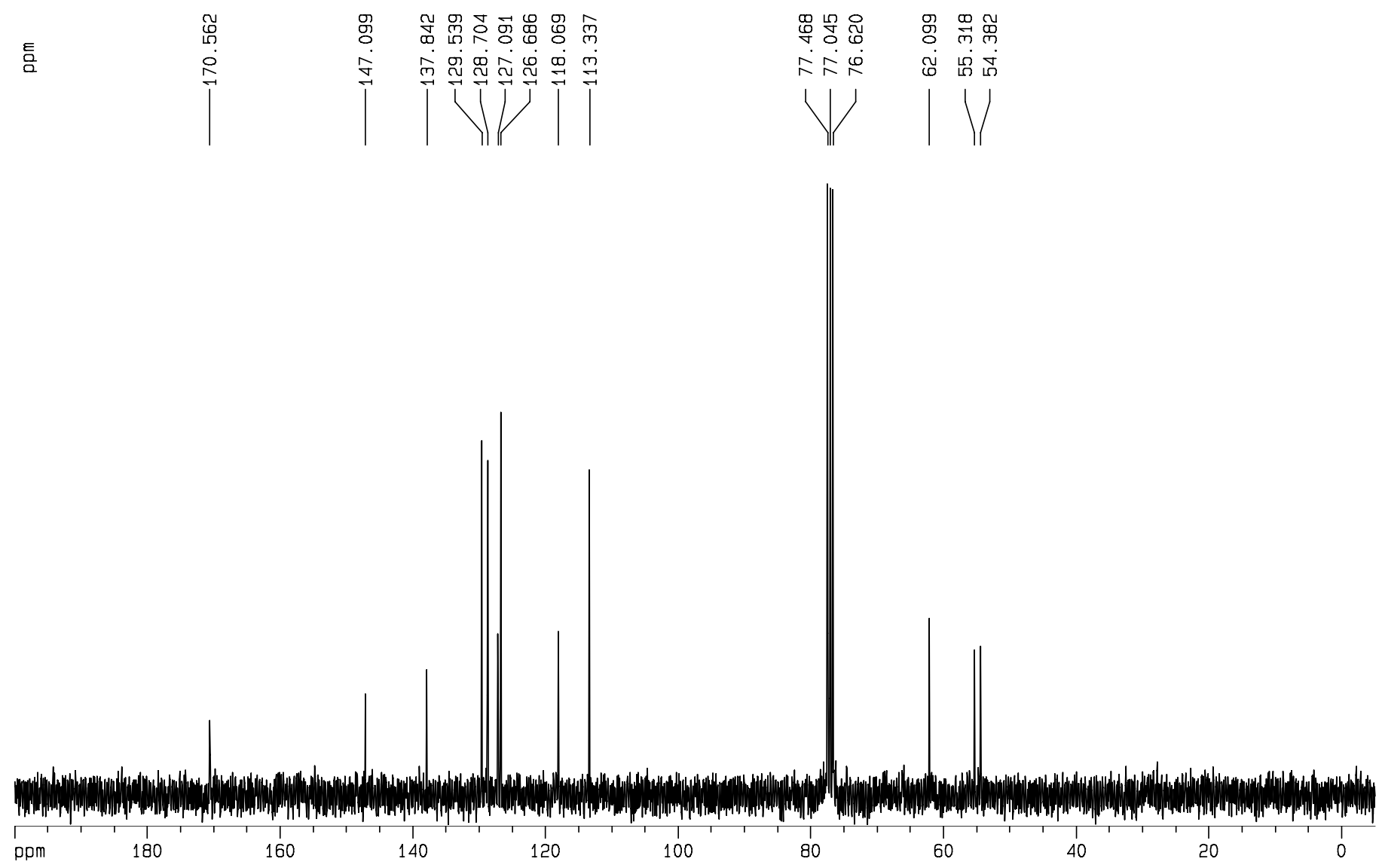

Current Data Parameters

NAME wjy06-0708b

PROCNO

F2 - Acquisition Parameters

Date
Time

PULPROG zgpg30

$\begin{array}{lr}\text { TO } & 65536 \\ \text { SOLVENT } & \text { COC13 } \\ \text { VS } & 48\end{array}$

4
4

=IDRES $\quad 0.274439 \mathrm{~Hz}$

AQ $\quad 1.8219508 \mathrm{sec}$

$\begin{array}{ll}{ }^{\mathrm{PG}} & 1824.6 \\ \mathrm{OW} & 27.800 \text { use }\end{array}$

$\begin{array}{cc}\text { JW } & 27.800 \text { usec } \\ \text { DE } & 5.00 \mathrm{usec} \\ \mathrm{TE} & 300.7 \mathrm{~K}\end{array}$

$\begin{array}{ll}\text { D1 } & 2.00000000 \mathrm{sec} \\ \mathrm{d} 11 & 0.03000000 \mathrm{sec}\end{array}$

$0.00002000 \mathrm{sec}$

$======$ CHANNEL $f 1======$
NUC1 $13 \mathrm{C}$

$\begin{array}{lr}P 1 & 9.40 \text { usec } \\ P L 1 & -1.00 \mathrm{oB} \\ \text { PL1 } & \end{array}$

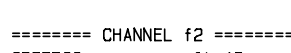

$\begin{array}{lr}\text { CPDPAG2 } & \text { waltz16 } \\ \text { NUC2 } & 1 \text { H }\end{array}$

$\begin{array}{ll}\text { PCPD2 } & 80.00 \mathrm{usec} \\ \text { PL2 } & -1.00 \mathrm{~dB}\end{array}$

$\begin{array}{ll}\text { PL12 } & 18.00 \mathrm{~dB} \\ \text { PL13 } & 18.00 \mathrm{~dB}\end{array}$

$18.00 \mathrm{~dB}$
$\mathrm{SF} 02$$\quad 300.1312005 \mathrm{MHz}$

F2 - Processing parameters
SI

$\begin{array}{lc}\text { SF } & 75.4677490 \\ \text { WOW } & \text { MHZ } \\ 5 S B & 0\end{array}$

$\begin{array}{lc}\text { SSB } & 0 \\ \angle B & 1.00 \mathrm{~Hz} \\ G B & 0\end{array}$

10 NMP plot parameters

$\begin{array}{ll}\mathrm{CX} & 22.00 \mathrm{~cm} \\ \mathrm{CY} & 10.00 \mathrm{~cm}\end{array}$

$\begin{array}{ll}F 1 \mathrm{P} & 200.000 \mathrm{ppm} \\ =1 & 15093.55 \mathrm{~Hz}\end{array}$

$\begin{array}{ll}F 2 \mathrm{P} & -5.000 \mathrm{ppm} \\ F 2 & -377.34 \mathrm{~Hz}\end{array}$

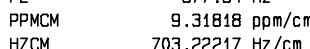


17- ${ }^{1} \mathrm{H}-\mathrm{NMR}$
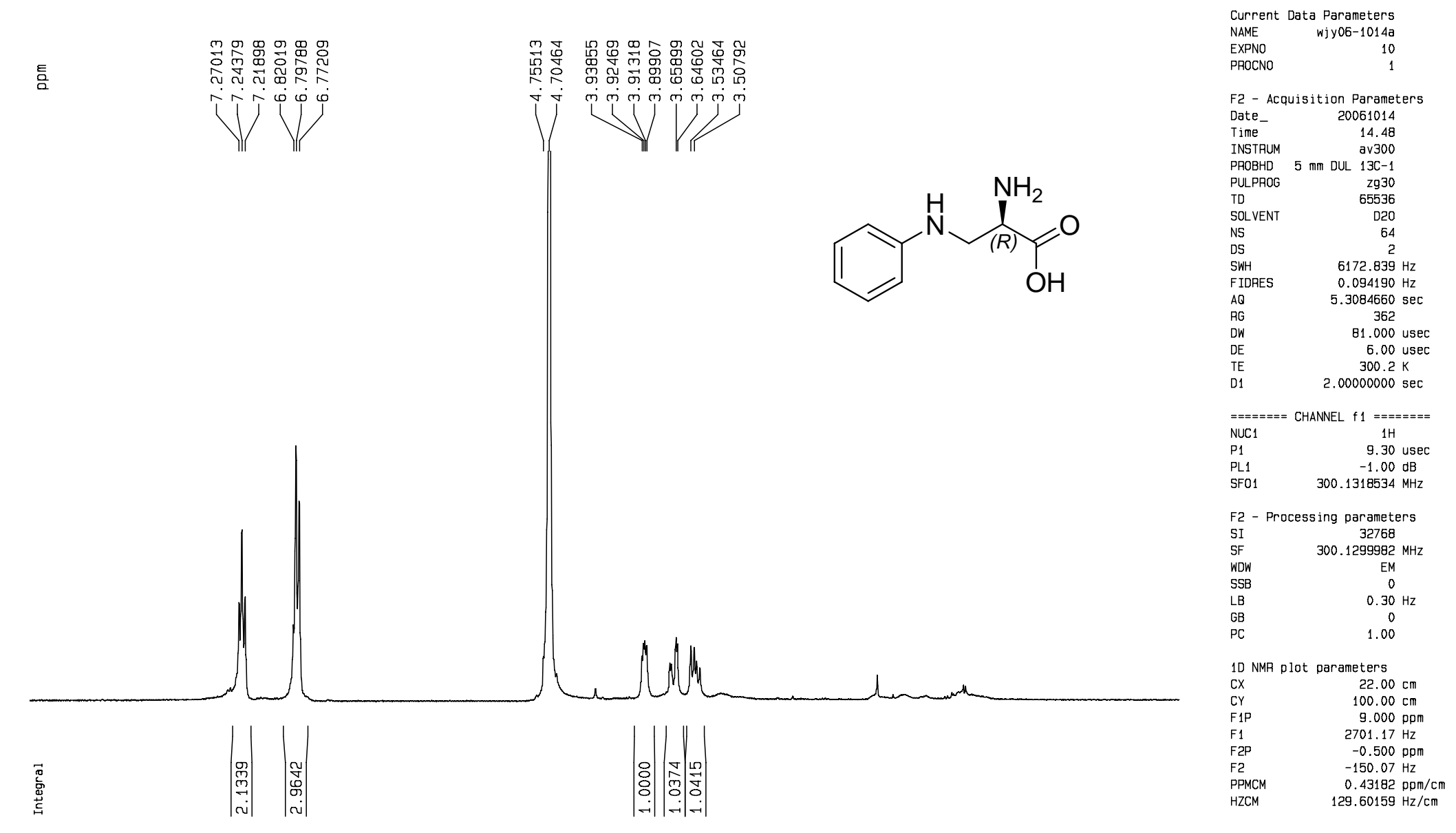
5. HPLC analysis of products. A Shimadzu LC-10AVP HPLC system was used to analyze enantiomeric excess values of all products.

Table S1: HPLC analysis of nitrile 1e, amides 2a-h and methyl esters 4a-h.

\begin{tabular}{cccc}
\hline Compound & $\boldsymbol{T}_{\mathbf{1}}(\mathbf{m i n})$ & $\boldsymbol{T}_{\mathbf{2}}(\mathbf{m i n})$ & Column \\
\hline $\mathbf{1 e}$ & 19.9 & 21.7 & Chiralcel ADH \\
$\mathbf{2 a}$ & 19.2 & 21.5 & Chiralcel ADH \\
$\mathbf{2 b}$ & 19.4 & 23.9 & Chiralcel ADH \\
$\mathbf{2 c}$ & 21.6 & 26.3 & Chiralcel ADH \\
$\mathbf{2 d}$ & 24.1 & 27.7 & Chiralcel OD \\
$\mathbf{2 e}$ & 26.8 & 29.3 & Chiralcel OD \\
$\mathbf{2 f}$ & 17.2 & 19.8 & Chiralcel OD \\
$\mathbf{2 g}$ & 15.6 & 16.9 & Chiralcel ADH \\
$\mathbf{2 h}$ & 18.2 & 19.7 & Chiralcel ADH \\
$\mathbf{4 a}$ & 12.4 & 13.8 & Chiralcel ADH \\
$\mathbf{4 b}$ & 18.3 & 22.5 & Chiralcel ADH \\
$\mathbf{4 c}$ & 12.9 & 16.7 & Chiralcel ADH \\
$\mathbf{4 d}$ & 13.3 & 16.9 & Chiralcel ADH \\
$\mathbf{4 e}$ & 17.7 & 22.1 & Chiralcel ADH \\
$\mathbf{4 f}$ & 12.1 & 14.1 & Chiralcel ADH \\
$\mathbf{4 g}$ & 14.9 & 16.0 & Chiralcel ADH \\
$\mathbf{4 h}$ & 13.0 & 13.9 & Chiralcel ADH \\
\hline
\end{tabular}

Chiralcel ADH employed hexane : isopropanol $=9: 1$ as mobile phase; flow rate $0.5 \mathrm{ml} / \mathrm{min} ; 25^{\circ} \mathrm{C}$.

Chiralcel OD employed hexane : isopropanol $=9: 1$ as mobile phase; flow rate $0.8 \mathrm{ml} / \mathrm{min} ; 25^{\circ} \mathrm{C}$. 
Table S2: HPLC analysis of diamino amides15a-d, 13, 14, and 17.

\begin{tabular}{cccc}
\hline Compound & $\boldsymbol{T}_{\mathbf{1}}$ (min) & $\boldsymbol{T}_{\mathbf{2}}$ (min) & Column \\
15a & 18.9 & 37.0 & Chiralcel ADH \\
15b & 30.0 & 50.4 & Chiralcel ADH \\
15c & 20.2 & 25.7 & Chiralcel ADH \\
15d & 23.0 & 32.3 & Chiralcel ADH \\
13 & 24.8 & 47.1 & Chiralcel ADH \\
14 & 27.6 & 28.8 & Chiralcel ADH \\
17 & 101.8 & 122.4 & Chirex(D)-Penicillamine \\
\hline
\end{tabular}

Chiralcel ADH employed hexane : isopropanol = $9: 1$ as mobile phase; flow rate $0.5 \mathrm{ml} / \mathrm{min} ; 25^{\circ} \mathrm{C}$.

Chirex(D)-Penicillamine employed $2 \mathrm{mM} \mathrm{CuSO}_{4}$ : isopropanol $=95: 5$ as mobile phase; flow rate $0.5 \mathrm{ml} / \mathrm{min} ; 25^{\circ} \mathrm{C}$.

\section{References}

(1) Rao, S. A.; Kumar, A.; Ila, H.; Junjappa, H. Synthesis, 1981, 623.

(2) Pereira, M. M.; Santos, P. P. O.; Reis, L. V.; Lobo, A. M.; Prabhakar, S. J. Chem. Soc.; Chem. Commun. 1993, 38.

(3) Kumar, H. M. S.; Rao, M. S.; Chakravarthy, P. P.; Yadav, J. S. Tetrahedron: Asymmetry 2004, 15, 127.

(4) Aires-de-Sousa, J.; Prabhakar, S.; Lobo, A. N.; Rosa, A. M.; Gomes, M. J. S.; Corvo, M. C.; Williams, D. J.; White, A. J. P. Tetrahedron: Asymmetry 2001, 12 , 3349. 